Portland State University

PDXScholar

Spring 1-1-2012

\title{
Development and Implementation of Acoustic Feedback Control for Scanning Probe Microscopy
}

Rodolfo Fernandez Rodriguez

Portland State University

Follow this and additional works at: https://pdxscholar.library.pdx.edu/open_access_etds

Part of the Atomic, Molecular and Optical Physics Commons, Diagnosis Commons, and the Medical Biophysics Commons

Let us know how access to this document benefits you.

\section{Recommended Citation}

Fernandez Rodriguez, Rodolfo, "Development and Implementation of Acoustic Feedback Control for Scanning Probe Microscopy" (2012). Dissertations and Theses. Paper 548.

https://doi.org/10.15760/etd.548

This Thesis is brought to you for free and open access. It has been accepted for inclusion in Dissertations and Theses by an authorized administrator of PDXScholar. Please contact us if we can make this document more accessible: pdxscholar@pdx.edu. 
Development and Implementation of Acoustic Feedback Control for Scanning Probe

Microscopy

by

Rodolfo Fernández Rodríguez

A thesis submitted in partial fulfillment of the requirements for the degree of

\section{Master of Science}

in

Physics

\section{Thesis Committee:}

Andres La Rosa, Chair

Raj Solanki

Erik Sanchez

Mingdi Yan

Portland State University

(C)2012 


\begin{abstract}
A remote-sensing acoustic method for implementing position control feedback in Scanning Probe Microscopy (SPM) is presented. The capabilities of this feedback control using the new Whispering Gallery Acoustic Sensing (WGAS) method is demonstrated in a Shear-force Scanning Probe Microscope that uses a sharp probe attached to a piezoelectric Quartz Tuning Fork (QTF) firmly mounted on the microscope's frame. As the QTF is electrically driven its mechanical response reaches the SPM frame which then acts as a resonant cavity producing acoustic modes measured with an acoustic sensor strategically placed on the SPM head. The novelty of the WGAS resides in using an SPM frame with a perimeter closely matching the intervening acoustic wavelength to act as a resonant cavity. The whispering gallery cavity constitutes an acoustic amplifier for the mechanical motion of the QTF probe. The observed monotonic behavior of the whispering gallery acoustic signal as a function of the probe sample distance is exploited here for tip-sample distance control with nanometer sensitivity, thus allowing topographic characterization as the probe is scanned across the sample's surface. This thesis includes a description of a Labview based programming for the Field Programmable Gate Array (FPGA) card used in the automated control of the WGAS feedback microscope, a solution for improving the effective resolution of the Digital to Analog Converter (DAC) and initial results towards theoretically modeling the WGAS working principle.
\end{abstract}




\section{Dedication}

This thesis is dedicated to my family. Through their lives I have understood my own. 


\section{Acknowledgements}

First and foremost I offer my sincerest gratitude to my supervisor, Dr Andres La Rosa, who has supported me throughout my thesis with his patience, knowledge and advice whilst allowing me the opportunity to work in his laboratory. I attribute the level of my Masters degree to his encouragement and effort and without him this thesis, too, would not have been completed or written. Throughout the years of research and physics courses here at PSU I have shared laboratory tasks with Xiaohua Wang and I am grateful for her support and help. I would also like to thank Mike Hopkins for his commitment to the laboratory projects. Special thanks to Keith Parker whose friendship and knowledge were a source of guidance. I would like to thank Philip Witham for his help and shared knowledge in handling the SEM. Thanks to a fine technician who kept machining parts for the different experiments, Marc Nisenfeld. The discussions with Derek Nowak were always fruitful and opened new avenues of thought. The support, help and advice of Erik Sanchez. I would also like to thank Nan Li for his help in the first stages of this work. Thanks to mi committee, Dr. Erik Sanchez, Dr Mingdi Yan and Dr Raj Solanki. Xin Wang, for his knowledge in preparing the necessary samples. Anne Keech and Kim Doty-Harris, who always managed to help with the orders. Thanks to the professors that have thought me during these years of learning, and especial thanks to Peter Leung for his tireless love of physics. Beyond Physics, AJ Lawrence, Mike DeArmond, Justin Dunlap and Mica Eastman have been of invaluable help and been good friends and sometimes managed to counter my introversion with the occasional happy hour. I am also indebted to the many countless contributors to the "Open Source" programming 
community for providing the tools and systems I have used to produce this thesis. The Physics Department at Portland State University has provided the support and equipment I have needed to produce and complete my thesis and funded my studies. Finally, I thank my parents for supporting me throughout all my studies. 


\section{Table of Contents}
ABSTRACT
i

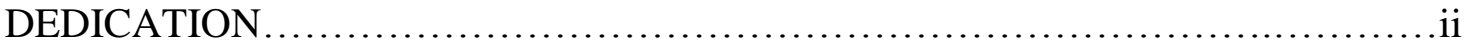

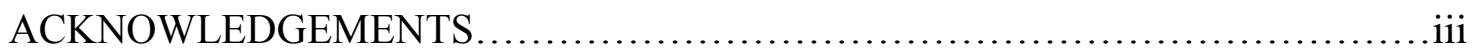

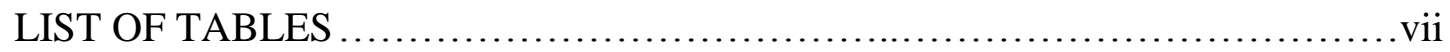
LIST OF

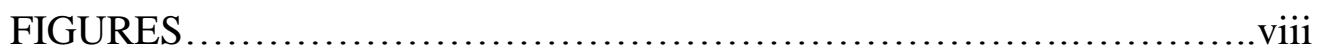
LIST OF
ABBREVIATIONS ..................................................

CHAPTER 1

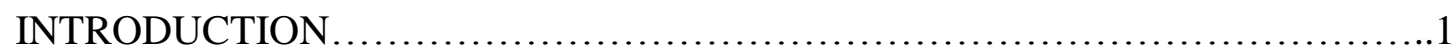

CHAPTER 2

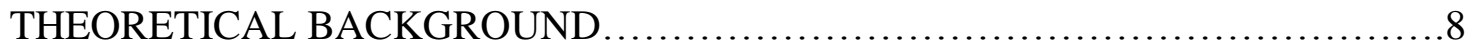

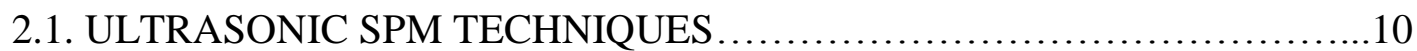

2.2. FORCED HARMONIC OSCILLATOR MODEL ........................... 14

2.3. NUMERICAL CALCULATION OF TIME RESPONSES .................18

2.4. FREQUENCY MODULATION TECHNIQUE ...........................29

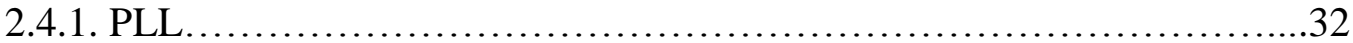

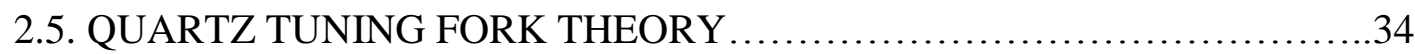

2.6. PIEZO STAGES ................................................ 40

2.7. THE FEEDBACK MECHANISM 41

CHAPTER 3

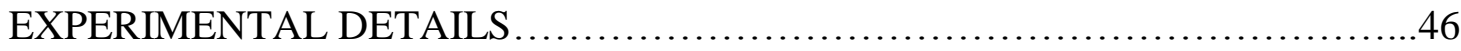

3.1. QUARTZ TUNING FORK PARAMETERS .............................47

3.2. XYZ PIEZO STAGE............................................... 50

3.3. PID CONTROL SOFTWARE ........................................ 52

3.3.1. PID ...................................................... 54

3.3.2. APPROACH AND RETRACTION CURVES PROGRAM.............58

3.4. HIGH RESOLUTION Z MOTION ..................................60

3.5. TIP PREPARATION ...............................................63 


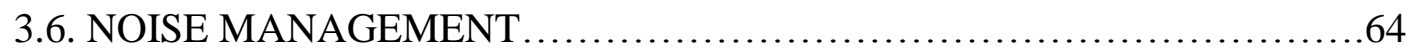

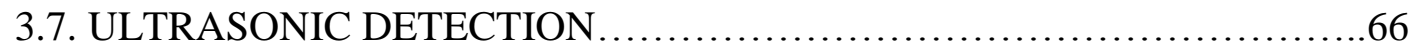

\section{CHAPTER 4}

ACOUSTIC RESONANT CAVITY SIMULATION ...............................71

4.1. FREQUENCY RESPONSE WHISPERING GALLERY ....................71

CHAPTER 5

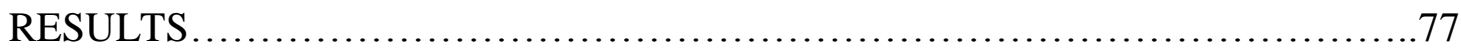

5.1. APPROACH AND RETRACTION CURVES ............................78

5.2. IMAGING WITH WGAS FEEDBACK............................... 88

CHAPTER 6

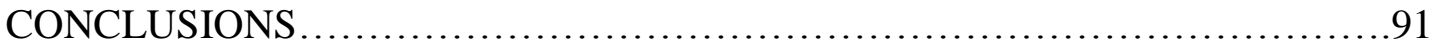

CHAPTER 7

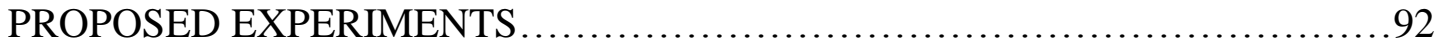

7.1. SUBSURFACE ULTRASONIC HOLOGRAPHY ......................93

7.2. INTEGRATED NEAR AND FAR FIELD MICROSCOPY ................95

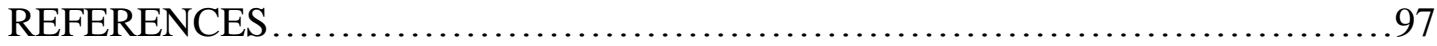




\section{List of Tables}

TABLE 1. TUNING FORK PARAMETERS FOR GOLD AND FIBER TIPS.......49 


\section{List of Figures}

FIGURE 1. BLOCK DIAGRAM OF A BASIC FEEDBACK LOOP. THE PID TRANSFER FUNCTIONS ARE ACTING SIMULTANEOUSLY ON THE ERROR SIGNAL AND THE OUTPUT IS FEEDING THE PROCESS, WHICH IN THE CASE OF SPM SYSTEMS CORRESPONDS TO THE PIEZOACTUATORS , QTF, SAMPLE AND THE TRANSDUCER..................................... 9

FIGURE 2. BASIC SETUP FOR THE STANDARD WHISPERING GALLERY ACOUSTIC SENSING. IT HAS A COMMON AFM SETUP WITH A COUPLE OF PIEZO MECHANICAL OSCILLATORS TO EXCITE THE SAMPLE ALONG THE SAMPLE PLANE OR PERPENDICULAR TO IT. THE MEASURED ULTRASONIC SIGNAL IS NOT USED FOR FEEDBACK PURPOSES. 11

FIGURE 3. BASIC SETUP FOR THE WHISPERING GALLERY FEEDBACK ULTRASONIC MicroscoPe. THE QTF-PROBE ATTACHED TO THE PZT IS MECHANICALLY COUPLED TO THE ACOUSTIC CAVITY THROUGH A MACOR HOLDER. ANY OSCILLATION OF THE QTF WILL GENERATE STANDING WAVES THAT WILL BE MEASURED BY THE USTS. THE INTERACTIONS OF THE PROBE WITH THE SAMPLE AFFECT THE QTF-PROBE OSCILLATION, AND THEREFORE THE ACOUSTIC SIGNAL MEASURED BY THE USTS.... 13

FIGURE 4. EXPERIMENTAL SETUP FOR SPM WITH WHISPERING GALLERY FEEDBACK. ..... 14

FIGURE 5. AFM TIP AND QTF TIP OSCILLATION..................................................... 15

FIGURE 6. GENERAL SOLUTION FOR THE FHO. ....................................................... 17

FIGURE 7. MODELED AMPLITUdE RESPONSE TO A FREQUENCY STEP OF 10 HZ.................. 19

Figure 8. MODELED PHASE RESPONSE TO A FREQUENCY STEP OF $10 \mathrm{HZ}$......................... 20

FIGURE 9. THE CHANGE IN THE POTENTIAL OF INTERACTION PRODUCES A CHANGE IN THE viii 
RESONANT FREQUENCY WHICH CHANGES THE AMPLITUDE OF OSCILLATION.

MATHEMATICALLY, THE PARAMETER THAT IS VARIED IS THE RESONANCE FREQUENCY.

FIGURE 10. DIFFERENCE BETWEEN QTF AND CANTILEVER OSCILLATION.

Figure 11. CALCUlation OF THE RESPONSE OF AMPLITUde, PHASE AND R FOR A PERIODiC INTERACTION. 23

FIGURE 12. TRANSFER FUNCTION FOR AM, PM, AND RM. THE QUALITY FACTOR IS 500 AND THE AMPLITUDE OF THE MODULATION IS $10 \mathrm{~Hz}$

FIGURE 13. TRANSFER FUNCTION FOR AM, PM, AND RM. THE QUALITY FACTOR IS 500 AND THE AMPLITUDE OF THE MODULATION IS $50 \mathrm{~Hz}$

Figure 14. TRANSFER FunCTION FOR AM, PM, AND RM. THE QUALITY FACTOR IS 2000 AND THE AMPLITUDE OF THE MODULATION IS $10 \mathrm{~Hz}$ 25

FIGURE 15. TRANSFER FUNCTIONS FOR Q OF 2000 AND 50 HZ MODULATION AMPLITUDE. 25

FIGURE 16. TRANSFER FUNCTIONS FOR Q OF 4000 AND HIGH MODULATION AMPLITUDES. 26

FIGURE 17. EXPERIMENTAL SUDDEN CHANGE IN RESONANT FREQUENCY. THE FREQUENCY SHIFTS TO ITS FINAL VALUE 10 MS AFTER THE INTERACTION. 27

FIGURE 18. EXPERIMENTAL SUDDEN CHANGE IN RESONANT FREQUENCY. THE AMPLITUDE CHANGES TO ITS FINAL VALUE 200 MS AFTER THE INTERACTION. 28

FIGURE 19. INTERPRETATION FOR THE SHIFT OF THE RESONANCE FREQUENCY AND THE AMPLITUDE DECREASE IN THE QTF SPECTRUM. 31

FiguRE 20. PHASE LOCK LOOP DIAGRAM. 32

FiguRE 21. RCL CIRCUIT EQUIVALENT TO THE MECHANICAL BUTTERWORTH-K. S. VAN DYKE QTF MODEL 35 


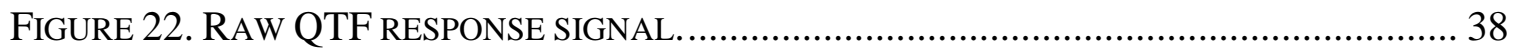

FIGURE 23. PROCESSED QTF RESPONSE SIGNAL ........................................................... 39

FigURE 24. FEEDBACK LOOP TRANSFER FUNCTION OF A GENERAL SPM............................ 42

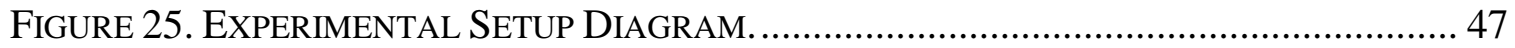

FIGURE 26. TUNING FORK OUTSIDE ITS ENCLOSURE, EXPOSED TO AIR. FIBER TIP MOUNTED

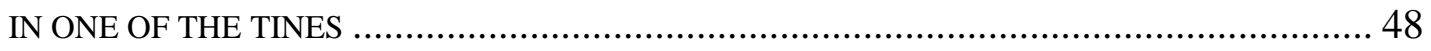

FIGURE 27. EXAMPLE OF FITTED QTF RAW DATA.......................................................... 48

TABLE 1. TUNING FORK PARAMETERS FOR GOLD AND FIBER TIPS ..................................... 49

FIGURE 28. QTF SPECTRUM BEFORE AND AFTER ADDING AN EQUIVALENT MASS ON THE

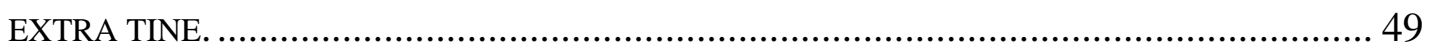

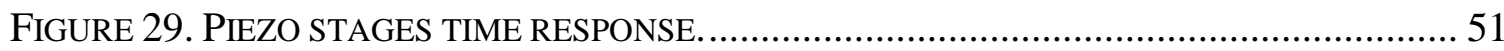

FIGURE 30. PID AND SCANNING LABVIEW CODE DIAGRAM........................................... 54

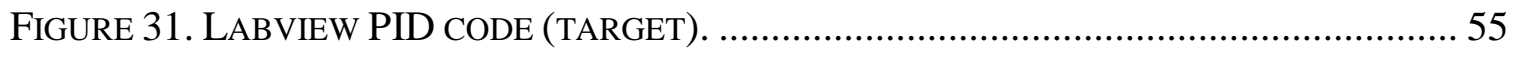

FIGURE 32. XY SCANNING LABVIEW PID CODE (TARGET). ……..................................... 56

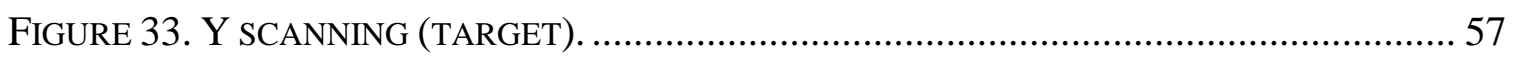

Figure 34. LabVIEW Front PANEL IMAging Control (Host). ..................................... 58

FIGURE 35. APPROACH AND RETRACTION PROGRAM BASIC LOOP CODE............................. 59

FIGURE 36. APPROACH AND RETRACTION PROGRAM HOST CODE. THE DIAGRAM SHOWS THE

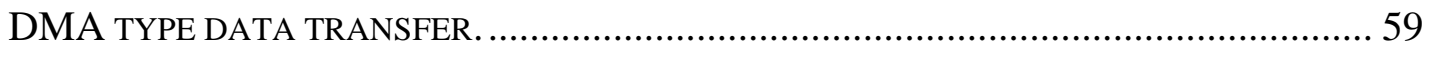

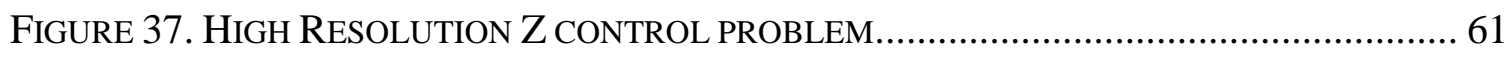

FIGURE 38. HIGH RESOLUTION Z CONTROL SOLUTION..................................................... 62

FigURE 39. OPTICAL FIBER TIP OBTAINED BY HF STATIC ETCHING................................... 64

FIGURE 40. A COPPER METALLIC MESH COMPLETELY SURROUNDS THE AL-EXTRUSIONS 
FRAME.

Figure 41. NOISE SPECTRUM BEFORE (LEFT) AND AFTER (RIGHT) THE FARADAY CAGE

IMPLEMENTATION 66

FIGURE 42. USTS HOLDER IMPLEMENTED TO IMPROVE CONTROL OF THE CONTACT

PRESSURE 67

FIGURE 43. FREQUENCY SWEEP USTS MEASUREMENT 68

FIGURE 44. COMPARISON BETWEEN THE SPECTRUM OF THE USTS AND THE QTF SIGNALS.

THERE IS A SHIFT OF 2 Hz IN THEIR RESONANT FREQUENCIES. 69

FIGURE 45. USTS MEASUREMENTS OF THE Front FACE OF THE WG............................ 70

FIGURE 46. SPM HEAD DESIGNED IN SOLIDWORKS................................................ 72

FIGURE 47. SPM HEAD WITH THE MESH PATTERN FOR SOLVING THE PDE EQUATION WITH THE COMSOL PACKAGE. ......................................................................... 73

FIGURE 48. SOLUTION FOR THE SPM HEAD STEADY STATE FREQUENCY RESPONSE AT 32 546 HZ. THE VALUES FOR INTENSITY ARE IN ARBITRARY UNITS. 74

FIGURE 49. FREQUENCY SPECTRUM OF A REGION FROM THE FRONTAL FACE OF THE

WHISPERING GALLERY. 75

FIGURE 50. COMSOL SOLUTION FOR THE SPM HEAD. SOLUTIONS FOR DIFFERENT POSITIONS ON THE CYLINDER’S SURFACE AT 0\%(TOP), 5\%(MIDDLE) AND 10\% (BOTTOM) INCREMENTS 76

FIGURE 51. APPROACH AND RETRACTION CURVE FOR A $45 \%$ OFFSET IN SILICON WITH A GOLD TIP. THE DEPTH OF THE LAYER FOR A $45 \%$ AMPLITUDE DAMPENING IS 5NM WHEREAS THE RETRACTION REQUIRES 18 NM TO ATTAIN THE MAXIMUM SIGNAL. ..... 79

FIGURE 52. A CLOSER LOOK AT THE APPROACH PROCESS OF FIGURE 51 80 
FIGURE 53. THE RETRACTION PROCESS OF FIGURE 52. THE SUDDEN CHANGE IN THE INTERACTION AFTER THE RETRACTION IS VERY WELL DEFINED. 81

FIGURE 54. APPROACH AND RETRACTION CURVE FOR A $45 \%$ OFFSET IN POLYMER WITH A GOLD TIP. THE FREQUENCY SHIFT CURVE HAS A TREND LINE INCLUDED TO SHOW THE OSCILLATIONS MORE CLEARLY. 82

FIGURE 55. THE APPROACH PROCESS. IT CONTAINS A REGION OF PURE DISSIPATIVE INTERACTION FOLLOWED BY ONE OF BOTH DISSIPATIVE AND CONSERVATIVE INTERACTIONS. 83

FIGURE 56. RETRACTION PROCESS. THIS REGION HAS THE SAME LENGTH AS OBSERVED OVER THE SILICON SAMPLE. 84

FIGURE 57. STEP MOTION OF TIP IN WATER LAYER. 85

FIGURE 58. QTF AMPLITUDE STABILITY ON SILICON EXPERIMENT. TWO DIFFERENT APPROACH PROCESSES ARE OBSERVED (ACCORDING TO THE AMPLITUDE CURVE)...... 86

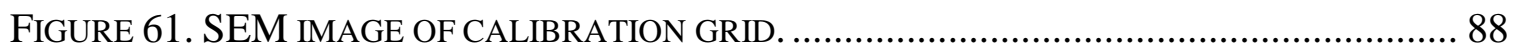

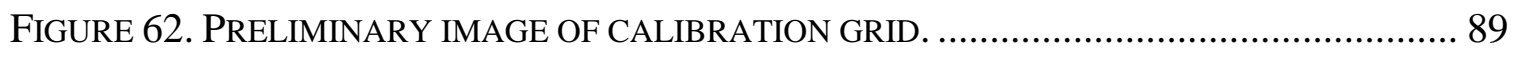

FIGURE 63. IMAGE OF CALIBRATION GRID USING WGAS FEEDBACK............................ 90

FIGURE 64. ULTRASONIC SUBSURFACE PRELIMINARY RESULT. ..................................... 94

Figure 65. INTEGRATEd NEAR AND FAR FIELd OptiCAL MiCRoscopy (INFO) PROPOSED TECHNIQUE. 95 


\section{List of Abbreviations}

\begin{tabular}{|c|c|}
\hline $\mathrm{ADC}$ & Analog to Digital Converter \\
\hline ADPLL & All Digital PLL \\
\hline AFAM & Atomic Force Acoustic Microscopy \\
\hline AFM & Atomic Force Microscopy \\
\hline $\mathrm{AM}$ & Amplitude Modulation \\
\hline $\mathrm{BNC}$ & Bayonet Neill-Concelman \\
\hline $\mathrm{CM}$ & Comsol Multiphysics \\
\hline CQO & Crystal Quartz Oscillator \\
\hline DAC & Digital to Analog Converter \\
\hline $\mathrm{DCO}$ & Digitally Controlled Oscillator \\
\hline DDS & Direct Digital Synthesizer \\
\hline DFT & Density Functional Theory \\
\hline DPLL & Digital Phase Lock Loop \\
\hline DSP & Digital Signal Processing \\
\hline DMA & Direct Memory Access \\
\hline EFGM & Electrostatic Force Gradient Microscopy \\
\hline EFM & Electrostatic Force Microscopy \\
\hline FEM & Finite Element Method \\
\hline FFT & Fast Fourier Transform \\
\hline FHO & Forced Harmonic Oscillator \\
\hline
\end{tabular}




$\begin{array}{ll}\text { FM } & \text { Frequency Modulation } \\ \text { FPGA } & \text { Field Programmable Gate Array } \\ \text { HF } & \text { Hydrofluoric Acid } \\ \text { INFO } & \text { Integrated Near Field Optical Microscopy } \\ \text { IC } & \text { Integrated Circuits } \\ \text { IPA } & \text { Isopropyl Alcohol } \\ \text { KPFM } & \text { Kelvin Probe Force Microscopy } \\ \text { LP } & \text { Low Pass Filter } \\ \text { MFM } & \text { Magnetic Probe Microscopy } \\ \text { NSOM } & \text { Near Field Scanning Optical Microscopy } \\ \text { PDE } & \text { Partial Differential Equations } \\ \text { PI } & \text { Proportional Integral } \\ \text { PID } & \text { Proportional Integral Derivative } \\ \text { PLL } & \text { Phase Lock Loop } \\ \text { PM } & \text { Phase Modulation } \\ \text { PVK } & \text { poly(N-vinylcarbazole) } \\ \text { PWM } & \text { Pulse Width Modulated } \\ \text { PZT } & \text { Piezo Tube } \\ \text { QTF } & \text { Quartz Tuning Fork } \\ \text { RF } & \text { Resonant Frequency } \\ \text { RLC } & \text { Resistance, Inductor and Capacitor } \\ \text { RMS } & \text { Root Mean Square } \\ \text { Real Part Modulation } \\ \text { Riv }\end{array}$


SHO Simple Harmonic Oscillator

SICM Scanning Ion Conductance Microscopy

SEM Scanning Electron Microscope

SAFM Scanning Acoustic Force Microscopy

SAM Scanning Acoustic Microscopy

SAW Surface Acoustic Wave

SNR Signal To Noise Ratio

SFM Shear Force Microscopy

SPM Scanning Probe Microscopy

STM Scanning Tunneling Microscopy

UAFM Ultrasonic Atomic Force Microscopy

UFM Ultrasonic Force Microscopy

USBS Ultrasonic Bottom Sensor

USTS Ultrasonic Top Sensor

VCO Voltage Control Oscillator

WG Whispering Gallery

WGAS Whispering Gallery Acoustic Sensing 


\section{Chapter 1}

\section{Introduction}

The invention of the STM by G. Binning and H. Rohrer in 1982 [1] opened the door for the development of a family of techniques known today as SPM. The STM's principle of operation rests on the quantum tunneling effect which takes place when a voltage biased sharp probe is brought close to a conductive surface. The spatial distribution of the tunneling current is continuously monitored as the probe scans across the surface providing tip-sample distance control. In 1986 the AFM [2] was developed broadening the range of samples from only conductive surfaces to encompass non-conductive samples, thus revolutionizing the investigation of local surface properties by bringing about the development of a whole new approach for measuring short-range chemical, van der Waals and electrostatic forces. Consequently, AFM spawned the development of unprecedented characterization methods that utilized the same basic SPM framework but which applied different physical principles of operation such as NSOM [5][6], MFM [7], AFAM, SICM, EFM [8] and EFGM [9], etc. Furthermore, the addition of dynamic control and the introduction of an instantaneous frequency tracker by Durig [10] allowed 
for harmonic analysis and tapping modes, thus increasing the analysis and characterization potential of SPM systems.

From the previous paragraph it can be inferred that the common denominator in SPM is the sharp probe placed in close proximity to the surface region under test, with an electronic system that carefully and continuously tracks the physical parameter of choice to control the tip-sample distance. As with any microscopy technique, several factors impact the image quality, reproducibility and resolution. The latter, for instance, which ranges from microns down to the atomic scale, depends on the tip geometry and the particular type of probe-sample interaction being exploited. In STM the tunneling current has an exponential relation with distance as well as a short range of interaction, in the order of Angstroms, resulting in the probe's closest atom to the surface being mostly responsible for the tunneling current. Contrarily, in the case of AFM the forces involved are usually comprised of interactions between several probe's atoms and the surface, because of the greater range of the forces involved. In principle, therefore, STM offers a better resolution than AFM, albeit only for conductive samples. Nevertheless, resolution may not be the primary focus of interest and the researcher may want to exploit another characteristic, depending upon the particular experiment being performed.

In the field of biology SPM techniques present the unique advantage of providing nanometer and sub-nanometer resolution with samples that are still 'alive', a feat that is not possible with SEM microscopy. This trait in conjunction with the non-invasive (and therefore nondestructive) character of the tapping or non-contact mode and its potential to 
characterize subsurface material properties, paramount in membrane biology, have made SPM's technically appealing for the development of acoustic based imaging and characterization. In particular ultrasound probing techniques have great potential in High Speed Biological Imaging [11]. Ultrasonic probing could overcome the barriers High Speed SPM techniques encounters not only with state-of-the-art electronics and PID feedback implementation but also with the invasive nature of contact force which compromises the integrity of the sample being imaged.

Furthermore, conventional ultrasonic techniques for characterizing materials have been widely used as imaging tools due to their non-invasive nature: an acoustic wave propagates through the sample and is modified both in its phase and amplitude without causing major disruption on the sample's structure, within a certain frequency range. It has found applications in medicine (medical ultrasonography, therapeutic ultrasound), construction (detection of fractures and dislocations in materials, material's thickness), mining, etc. Ultrasonic imaging based on transmission and reflection of ultrasonic waves was first implemented in 1974, and it was named SAM [12]. Several years later, the wave of SPM based techniques also included ultrasonic techniques such as UFM [13][14], SAFM [16], AFAM [15] and UAFM [17]. For a SPM based ultrasonic technique, the local resolution can be improved if an acoustic wave is guided towards the sample by a structure smaller and closer to the surface than the acoustic wavelength [18]. This constitutes the near-field principle for acoustic waves, having as a consequence the dependence of the microscope's resolution on its operating frequency. At room temperature and at an operating frequency from tens of megahertz to several gigahertz, 
the acoustic microscope resolution ranges from hundreds of microns to tenth fractions of a micron. With superfluid helium as an immersion liquid, the operating frequency of a cryogenic acoustic microscope rises to tens of gigahertz, and its resolution to tens of nanometers.

The acoustic feedback technique developed in this thesis constitutes a continuation of a pioneering effort made by our research group to introduce acoustic sensing into the feedback mechanism of scanning probe microscopes [3] [4]. It started in 2004 when it was discovered that the lateral oscillation of a sharp probe, while being immersed into the water-film layer typically found adsorbed to any surface at ambient conditions, engenders acoustic waves which can be sensitively detected by a commercially available acoustic transducer. Furthermore, an energy balance analysis showed a mismatch between the energy received by the acoustic sensor and the energy lost by the probe. The obvious conclusion for the given experimental setup was that such energy difference was traveling upwards, through the PZT, towards the SPM head frame. Undoubtedly, such a transfer would make the energy widely dispersed making detection enormously difficult. Not surprisingly, a first attempt at measuring the signal showed a very low SNR. The fact that the acoustic wavelength in stainless steel is approximately $15 \mathrm{~cm}$., very close to the microscope's head perimeter, lead to the assumption of standing waves formation and the existence of an acoustic eigenfrequency close to the resonant frequency of the QTF. As a side note, a similar situation is observed in cathedrals were a feeble whispering can be heard at a distance; hence the name Whispering Gallery Feedback. Finally, we proposed that the microscope frame itself performs as a resonance cavity, sensitively detecting the 
mechanical motion of the QTF firmly attached to the microscope stage. Since it is well documented that shear force interactions between the probe and the sample drastically affects the mechanical motion of the QTF it followed that the acoustic sensor (via QTF coupling) could also monitor monitor them. The natural consequence of this earlier work was the conjecture that a system could be designed to improve the signal by taking advantage of the resonant frequencies of the acoustic resonance cavity. To place these ideas in more solid grounds, a series of simulations where run carefully replicating the experimental settings of the WG in order to shift its resonant frequencies around the range of interest. To sum up, those were the beginnings of our attempts to introduce acoustic signals in feedback systems in the context of near-field scanning optical microscopy development. The main purpose of the present thesis is to show how to exploit these acoustic phenomena, i.e. the ability of an acoustic resonant cavity to sensitively monitor the mechanical motion of a tuning fork affected by the shear forces between the probe and the sample, for probe-sample distance control. The advantages found are: direct tracking of the QTF's motion, potential for improved signal-to-noise ratio and the perspective for implementing holography interferometric techniques. In addition, the distinctions between the WGAS feedback and other types of acoustic scanning probe microscopes will be presented.

Chapter 1 offers a brief introduction to Scanning Probe Microscopy and its impact as analytical techniques for supporting characterization, nanotechnology developments and miniaturization.

Chapter 2 includes the theoretical background, describing the working principle of the 
different components, numerical calculations of the signals with potential for feedback control, such as AM, PM and RM, analysis of the Damped Forced Simple Harmonic Oscillator model to describe the QTF's motion and interactions. The feedback conditions and the parameters for improving it in terms of stability and time response are also analyzed.

Chapter 3 describes the experimental setup, the steps taken to improve the SNR of the system (signal conditioning implementation). Tip preparation techniques used are also reviewed. We also analyze the different parameters necessary to obtain a stable feedback mechanism and the role of frequency modulation and phase modulation.

Chapter 4 presents computer simulations aiming at understanding the working principle of the ultrasonic imaging technique and, thus allowing to optimize the strategies for increasing the detection efficiency of the ultrasonic signal. It order to obtain a better SNR it was proposed that the diameter of the cavity should be modified to have the structure peaked at the same resonance frequency of the tuning fork. Based on this assumption, several simulations were carried out using a Finite Element Method software package (Comsol Multiphysics). The information of the intensity for different frequencies was used to assemble a series of frequency response graphs that allowed to predict the proper dimensions for a matching resonance frequency. This information together with the QTF spectra permits the design of a future WGAS.

Chapter 5 presents the experimental results, including a series of approach and retraction curves using FM to decouple the interaction forces between tip and sample; and images 
obtained with the WGAS feedback technique.

Chapter 6 offers the conclusions of this work.

Chapter 7 outlines a series of proposals for potential experiments for the SPM with WGAS setup already assembled. 


\section{Chapter 2}

\section{Theoretical Background}

A distinctive characteristic of SPM techniques is their control of the probe-sample distance. Since they are intended to measure near field interactions SPM systems use feedback mechanisms, such as the one showed in figure 1, to maintain a nanometric distance between the probe and the object under study. A transducer, which transforms a physical property into an electrical signal, continuously tracks a given physical parameter providing the input signal for the feedback loop. A change in the physical property, the result of the interaction between probe and sample, correspond to a change in the electrical signal. The controlling parameter is extracted from this signal (as we will see below it could be the signal's Amplitude, Phase, Resonant Frequency for oscillating probes, or the signal itself in the case of unmodulated interactions) and subtracted from a given reference value (see figure 1). The result is called the error signal and it feeds the PID controller, the most widely utilized feedback mechanism in SPM, which is permanently trying to keep it as close to zero as possible. 


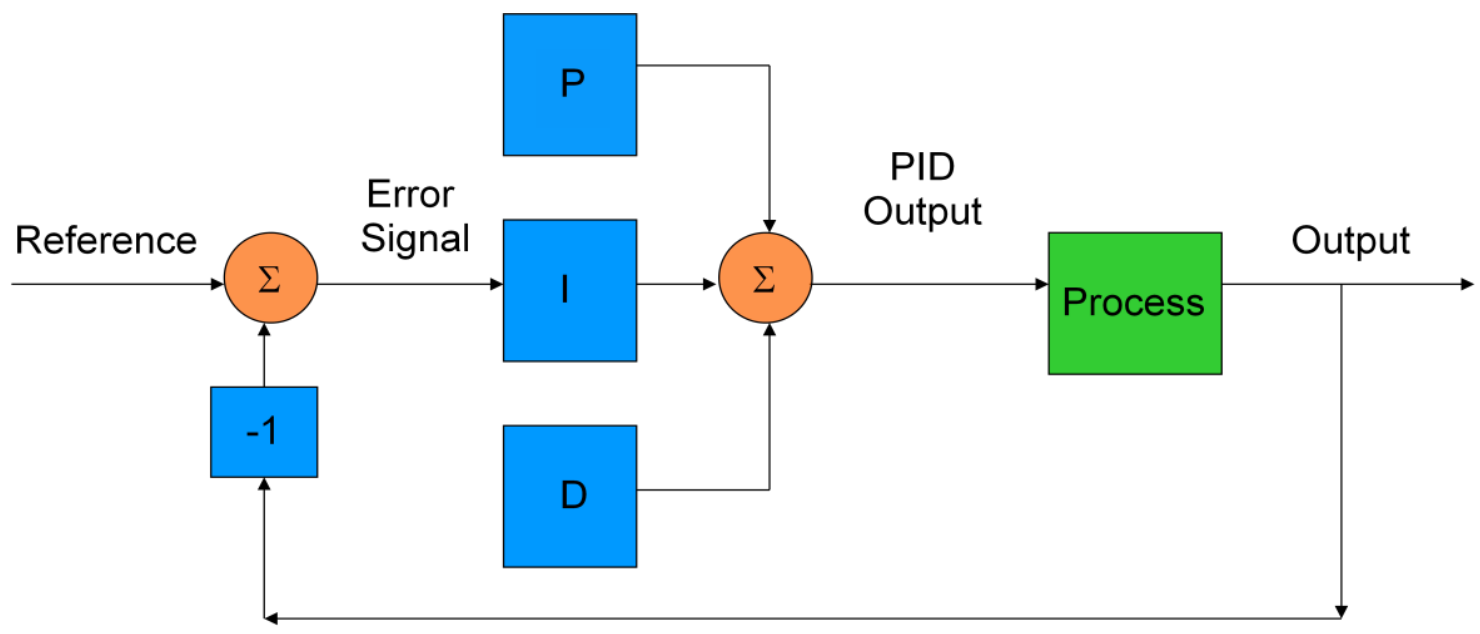

Figure 1. Block diagram of a basic feedback loop. The PID transfer functions are acting simultaneously on the error signal and the output is feeding the process, which in the case of SPM systems corresponds to the piezoactuators, QTF, sample and the transducer.

Each one of the PID terms produce specific responses to the input. The proportional term of the PID reacts to any instantaneous variation of the error signal, the integral term is proportional to the accumulated error, and the derivative term reacts to the rate of change of the error signal. Repeated experiments showed us that the derivative part of the PID introduces unwanted instabilities in the feedback control. As a result, in this implementation the Derivative term was left out, as it is in most commercial SPM systems.SPM techniques are dissimilar only in the monitored physical property used to control the tip sample distance and the technique employed to acquire it. In the case of a force based SPM, optical detection has the advantage of being very sensitive and having a large SNR compared to other techniques. However, it has the disadvantage of involved implementation, requiring optical alignment and calibrated optics. An alternative solution was available with the introduction of QTF - a type of CQO with piezoelectric properties 
as a cheap and robust transducers for tip-sample distance control in NSOM. By mounting the probe on one of the tines, exciting the QTF and measuring its electrical response to the tip sample interaction the distance could be monitored.

When a oscillating voltage is applied to the QTF, the design of the electrical connections on it excite only the symmetrical mode of oscillation [28]. Any variation in the amplitude (controlling parameter) or motion-related signal, such as phase or resonant frequency shift, is interpreted by the feedback system as an interaction of the probe with the water layer (or the sample) causing it to take action in order to maintain the controlling parameter at a predetermined level (error signal equal to zero).

In this chapter we describe the theoretical background for the calculation of the feedback parameters, starting with clarifying the difference between the WGAS technique and other known ultrasonic SPM techniques.

\subsection{Ultrasonic SPM techniques}

Ultrasonic waves are defined as any mechanical wave with a frequency above $20 \mathrm{KHz}$ (above the limit of human hearing). Abbe's principle states that focused waves are restricted in their lateral resolution to about half a wavelength. Higher excitation frequencies produce smaller wavelengths, increasing the resolution. To improve 
resolution ultrasonic techniques, specifically Near-Field Acoustic techniques, frequencies in the order of $\mathrm{MHz}$ and $\mathrm{GHz}$ are usually employed. The first ultrasonic imaging technique for characterizing materials was developed in 1974 and was called SAM [12]. SAM works by detecting reflected, absorbed, transmitted or scattered sound waves produced by the interaction between induced vibrations and the sample.

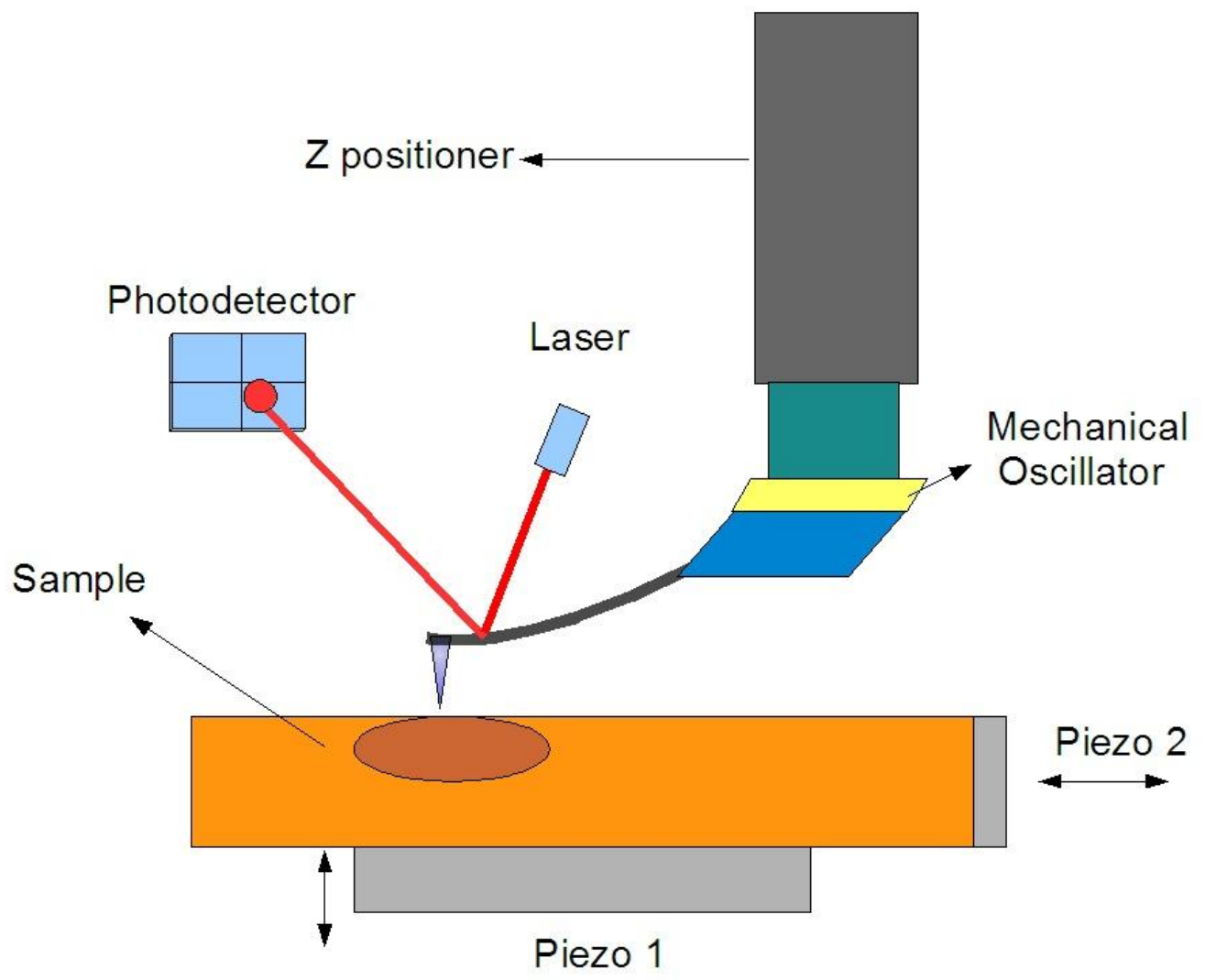

Figure 2. Basic setup for the standard whispering Gallery Acoustic Sensing. It has a common AFM setup with a couple of piezo mechanical oscillators to excite the sample along the sample plane or perpendicular to it. The measured ultrasonic signal is not used for feedback purposes.

After AFM appeared, several ultrasonic based techniques were developed: UFM [13][14], where a MHz signal with $\mathrm{kHz}$ modulation is applied to the sample and picked 
up by the AFM cantilever, through the mechanical diode effect; U. Rabe et. al. introduced the AFAM [15] technique where the sample is mechanically excited with longitudinal and transversal waves which are picked up by the AFM cantilever working in contact mode; SAFM [17], where two propagating surface acoustic waves interfere and are detected by an AFM cantilever also in contact mode. Finally, UAFM [17] where the cantilever is excited in the MHz range and scanned across the sample in contact mode.

Currently, most ultrasonic SPM techniques are based on the setup showed in figure 2 . The sample is placed on piezoelectric materials which generate the oscillations that will interact with the ones originating on the probe. The resulting interaction produces wave interference patterns and depending on the direction and frequency of these traveling soundwaves the cantilever will be excited at specific modes, thus rendering valuable and unique information from the sample. However, these signals are used for recording purposes only and none of them are used for feedback control.

Unlike the aforementioned setups, the mechanism proposed in this thesis employs an ultrasonic signal (in the range of $32 \mathrm{kHz}$ ), originated in the vibration of the QTF and amplified by the resonant cavity, as the signal fed to the PID control. Whether it is mechanical or electrical excitation, this setup provides two main advantages with respect to common QTF techniques, a) direct measurement of the mechanical response of the QTF, which is not possible when the signal is measured electrically, as shown in section 5 , and b) the resonant cavity can be designed to have resonant modes coinciding with the resonant frequency of the QTF, increasing the SNR of the system to match or even surpass the current state of the art SPM QTF system. Figures 3 and 4 show the setup and 
the feedback control diagrams, respectively.

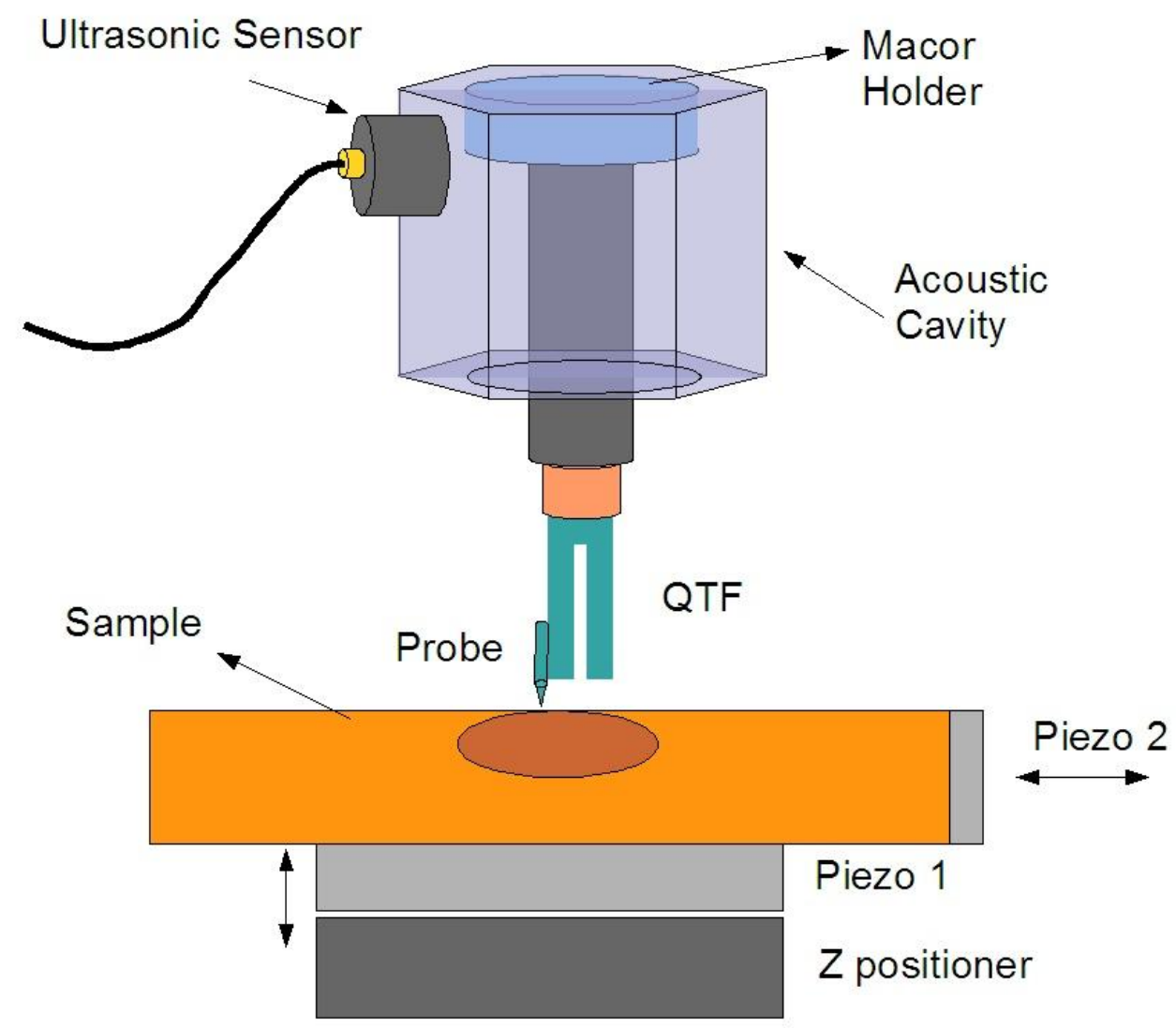

Figure 3. Basic setup for the Whispering Gallery Feedback Ultrasonic Microscope. The QTF-probe attached to the PZT is mechanically coupled to the acoustic cavity through a Macor holder. Any oscillation of the QTF will generate standing waves that will be measured by the USTS. The interactions of the probe with the sample affect the QTF-probe oscillation, and therefore the acoustic signal measured by the USTS.

A description of the model used to describe the motion of the QTF is the subject of the following section. 


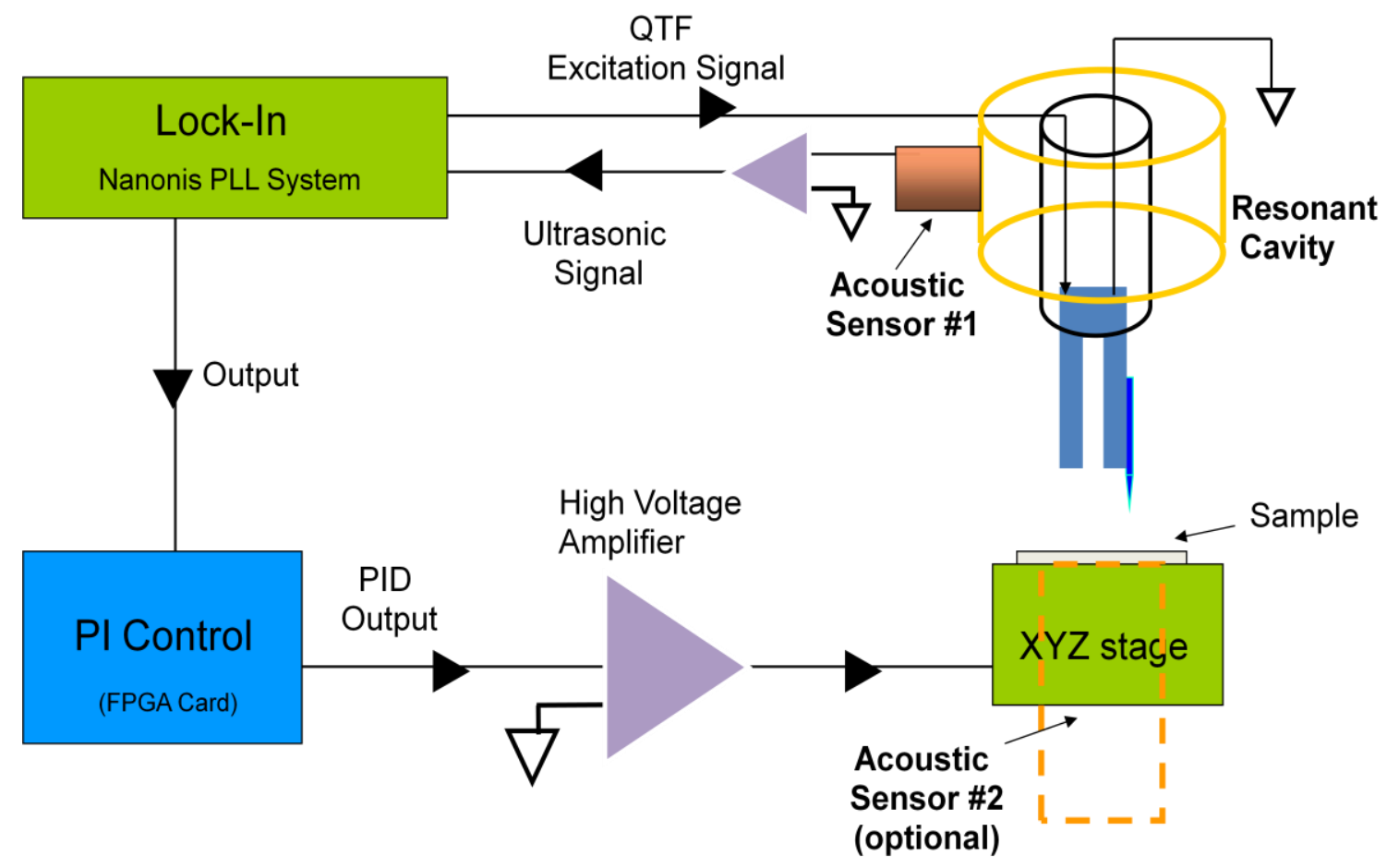

Figure 4. Experimental setup for SPM with Whispering Gallery Feedback.

\subsection{Forced Harmonic Oscillator Model}

Initially introduced by Gäunther, Fischer and Dransfeld [19] and later developed by Karrai and Grober [20], the CQO (XY cut) has been used for NSOM tip-sample positioning control for quite some time [21][22][23]. The theoretical description often used to account for the motion of the QTF is given by the FHO model. The FHO model provides an excellent qualitative and good quantitative approximation for the motion of the QTF in the absence of first principles calculations. The equation of motion is given by 


$$
\ddot{x}+2 \beta \omega_{0} \dot{x}+\omega^{2} x=\frac{F_{0}}{m} \cos \omega_{d} t+F_{\text {int }},
$$

where $x$ is the tip's position, $\beta$ is the damping ratio, $\omega_{0}$ is the frequency of oscillation, $F_{0}$ is the driving force, $m$ is the equivalent mass of both the QTF and the tip, $\omega_{d}$ is the driving force angular frequency and $F_{\text {int }}$ is the interaction force. The dot above a variable indicates total differentiation with respect to time. The $\beta$ coefficient encompasses all of the dissipative interactions whereas the interaction force $F_{\text {int }}$ encircles every conservative interaction on the tip. A further discussion of these interactions will be carried out in section 4.

The simplest FHO model accounts for only one dimensional motion and it is used to describe the motion and interactions of the a probe while it oscillates perpendicular (AFM) or parallel (SFM) to the sample's surface (figure 5).

Cantilever AFM tip

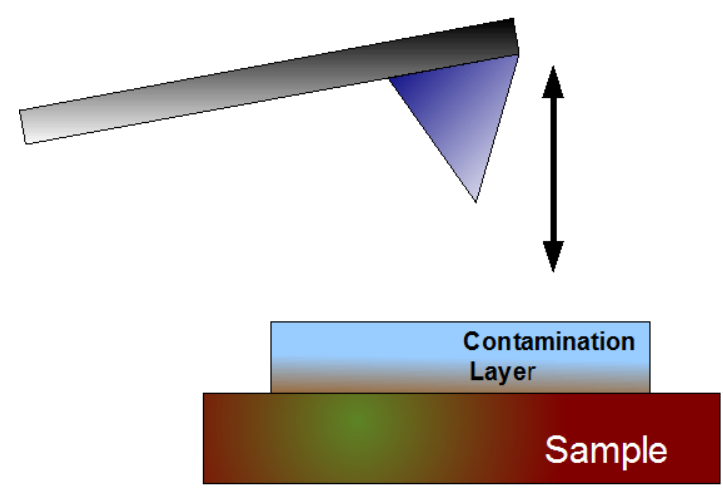

Quart Tuning Fork tip

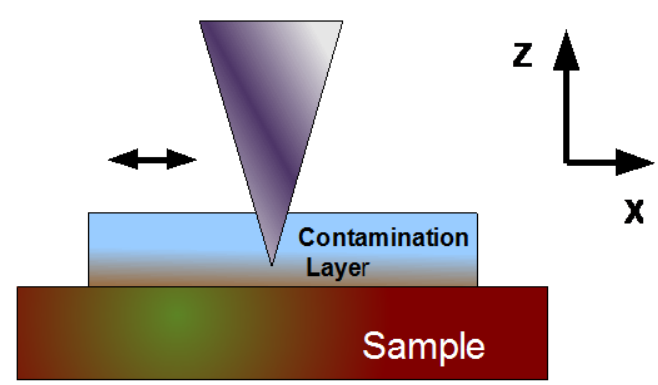

Figure 5. AFM tip and QTF tip oscillation. 
A general expression for $F_{\text {int }}$ is found by expanding $x$ around $x=x_{0}$ and assuming small oscillations approximation $\left(\frac{\partial F}{\partial x} \neq 0\right)$,

$$
F_{i} n t \approx\left({\frac{\partial F_{i n t}}{\partial x}}_{x_{0}}\right) x
$$

where $x_{0}=0$ is the equilibrium position. Plugging equation (2) into (6) we obtain

$$
\ddot{x}+2 \beta \omega_{s} \dot{x}+\omega_{s}^{2} x=\frac{F_{0}}{m} e^{i \omega_{d} t}+\frac{\partial F_{\text {int }}}{\partial x} x .
$$

After rearranging the terms of the last equation, we can write,

$$
\ddot{x}+2 \beta \omega_{s} \dot{x}+\left(\omega_{s}^{2}-\frac{\partial F_{\text {int }}}{\partial x}\right) x=\frac{F_{0}}{m} e^{i \omega_{d} t}
$$

where

$$
\omega_{s}^{2}=\omega_{d}^{2}+\frac{\partial F_{i n t}}{\partial x_{x_{0}}}
$$

Note that what causes the frequency change is not a variation of the absolute value of the force but of its derivative with respect to the position. Applying the complex variable method changes the equation to

$$
\ddot{X}+2 \beta \omega_{0} \dot{X}+\left(\omega_{s}^{2}-\frac{\partial F_{\text {int }}}{\partial X}\right) X=\frac{F_{0}}{m} e^{i \omega_{d} t},
$$

where $X$ is now a complex variable. We now look for a the solution of the form 


$$
X(t)=\bar{X} e^{i \omega_{d} t},
$$

where $\bar{X}$ is a complex constant. The motion would be described by the real part of $X$ but since we are only concerned with the amplitude of the oscillation, we solve for $\bar{X}$ by plugging (7) it into (4), simplifying the exponential terms, and solving the resulting algebraic equation, thus obtaining

$$
\begin{array}{r}
\bar{X}(t)=D\left(\omega_{s}\right) e^{-\beta \omega_{s} t} e^{i\left(\omega_{s} \sqrt{1-\beta^{2}}-\omega_{d}\right) t} \\
+\frac{F_{0} / m}{\omega_{s}^{2}-\omega_{d}^{2}+2 \beta \omega_{d} \omega_{s}},
\end{array}
$$

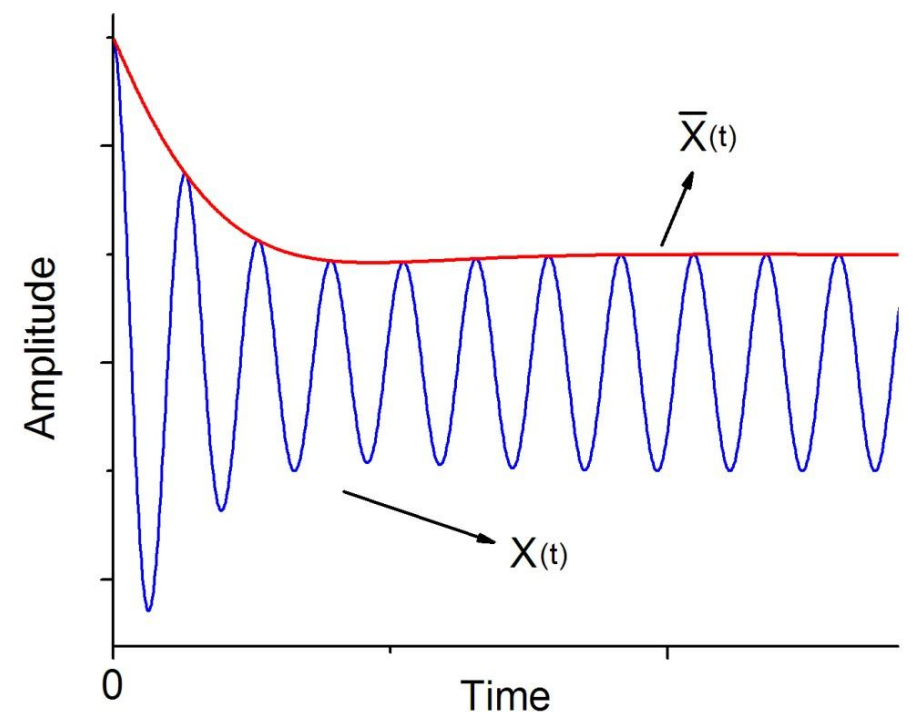

Figure 6. General solution for the FHO. 
where $D\left(\omega_{s}\right)$ is a complex term. It should be clear that equation (8) gives the instantaneous amplitude variation, i.e the wave's envelope.

The first term on the right hand side will decay very rapidly due to the exponential term, accounting for the transient part of the QTF's motion. The second term is the very well known steady state solution. In the next section we will obtain valuable information from this equation by interpreting the transient and steady state solutions.

\subsection{Numerical Calculation Time Responses}

In the previous section it was showed that the QTF's motion is described by equation (8), presenting a transient and a stationary part. The amplitude of the QTF's oscillation is constrained between two values, $A_{i}$ and $A_{f}$. $A_{i}$ is determined by the initial conditions and $A_{f}$ by the stationary state. The absolute change in amplitude (i.e. $A_{f}-A_{i}$ ) depends on both the interaction potential and the viscosity of the medium. The small oscillations approximation gives the interaction force $F_{\text {int }}$ as described in equation (2). A change in $\frac{\partial F_{\text {int }}}{\partial X}$ produces a change of the resonant frequency as showed in (5).

A closer look to equation (8) reveals the transient part as a function of the resonant frequency $\omega_{s}$ as well as of the damping ratio $\beta$, whereas the steady state depends on $\omega_{s}$ 
alone. It is worth noting that the damping ratio is related to the quality factor $Q$ by

$$
Q=\frac{1}{2 \beta}
$$

To test the model the time response of the system is plotted when the change in $\omega_{s}$ is 10 $\mathrm{Hz}$, which is a common value found in experimental conditions. In addition, the QTF's resonant frequency of $32,767 \mathrm{kHz}$ is used as the value for $\omega_{d}$ and the Q varies from 1000 to 4000 in steps of 1000 . Figure 7 shows the motion's amplitude, $\|\bar{X}\|$, versus time.

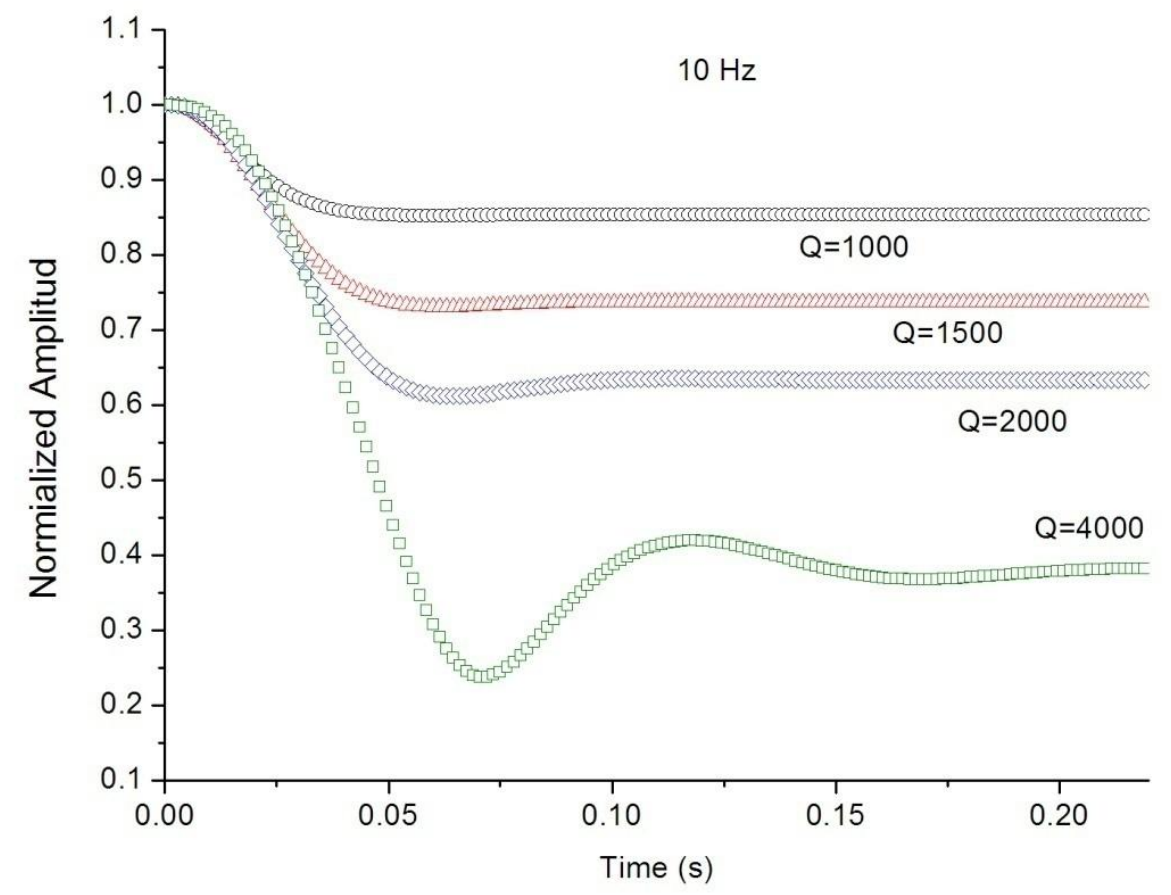

Figure 7. Modeled amplitude response to a frequency step of $10 \mathrm{~Hz}$.

Not surprisingly, the amplitude of the final state is proportional to $\mathrm{Q}$ and, by definition, inversely proportional to $\beta$. The time response to this force step significantly decreases. 
At first glance this is not evident with a smaller Q. 8 presents the motion's phase, $\arg \bar{X}$, which has a similar behavior. The sign of the phase is arbitrary.

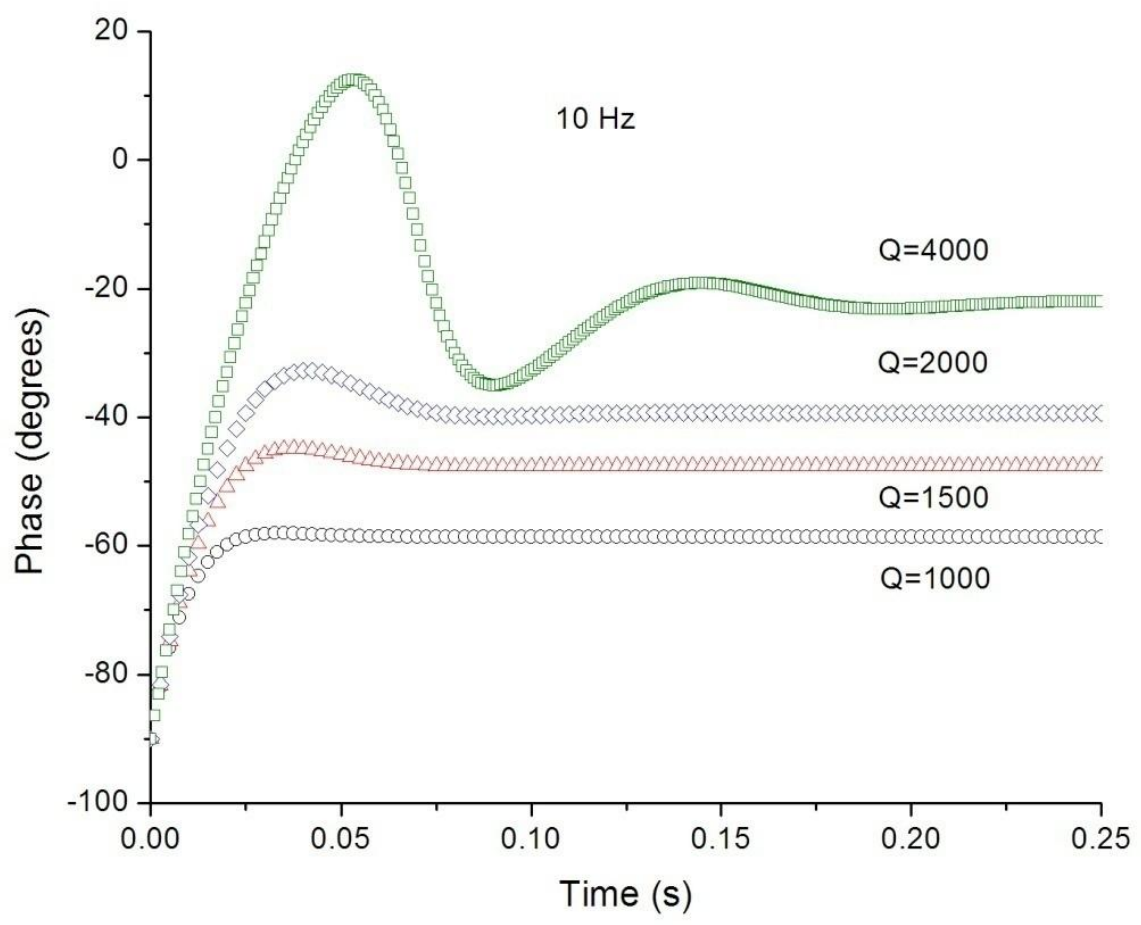

Figure 8. Modeled phase response to a frequency step of $10 \mathrm{~Hz}$.

A one-time interaction change allows the system to achieve the final steady state amplitude. However, real experimental interactions are rarely a onetime phenomenon. To simulate subsequent changes in the interaction potential the parameter $\omega_{s}$ isvaried to represent such interaction changes. In other words, it is possible to numerically simulate this situation by plotting the motion for two different resonant frequencies, one after the other. This procedure is schematically shown in figure 9 . The motion is modulated by a second signal which is produced by the change in the potential interaction causing a shift 
in the resonant frequency. When the frequency of this modulation is zero we have the situation shown in 7.

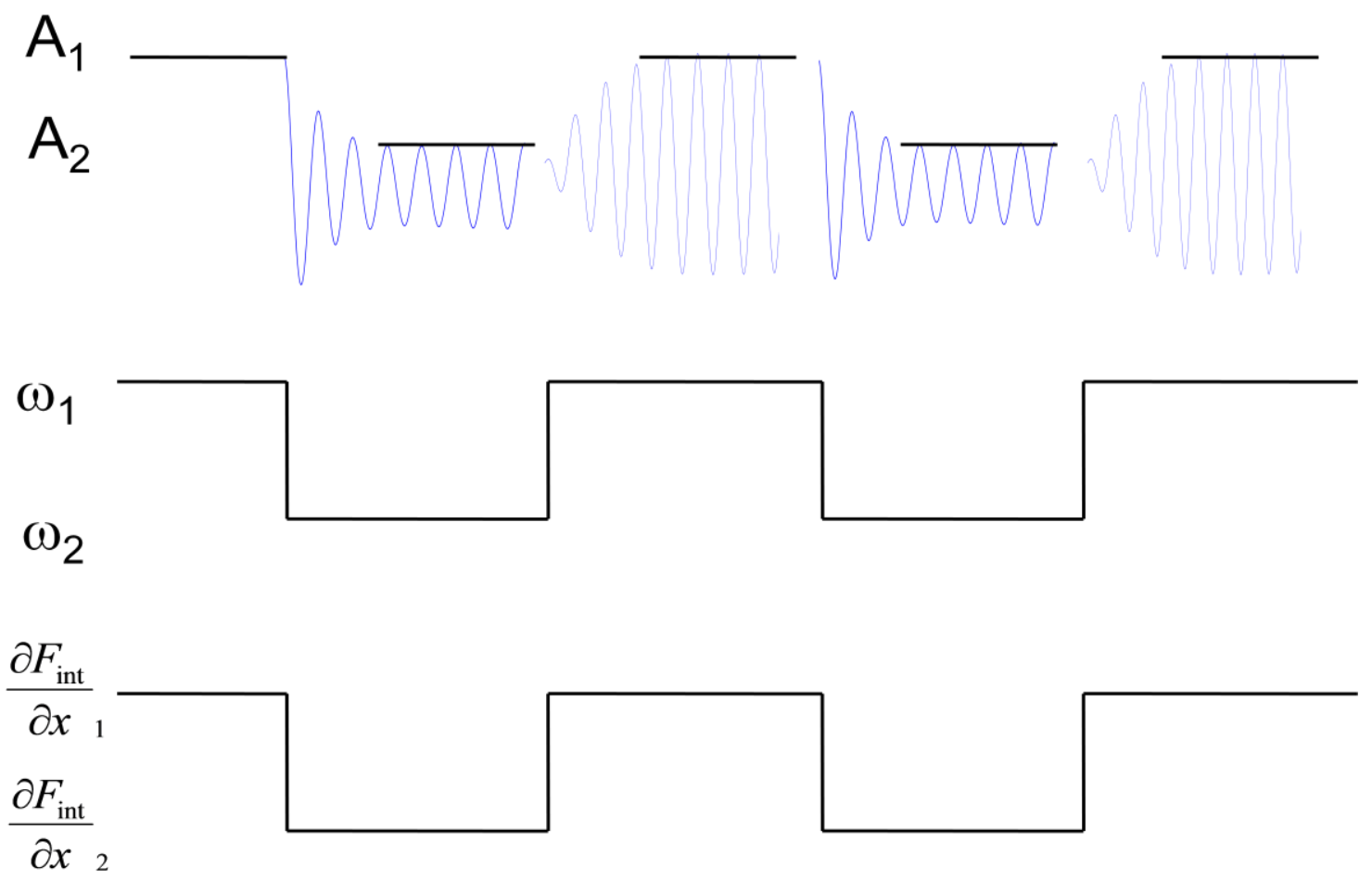

Figure 9. The change in the potential of interaction produces a change in the resonant frequency which changes the amplitude of oscillation. Mathematically, the parameter that is varied is the resonance frequency.

As the frequency increases the gain of the system its ability to respond to different interaction potentials will decrease because an increase of the modulation's frequency precludes the system to ever reach the steady state. From this point on we will refer to this change of the interaction potential as a change in the resonance frequency $\omega_{s}$, since they are both connected through equation (5). If we plot the normalized amplitude response versus the modulation's frequency we obtain the bandwidth of the system [39]. 
Experimentally this will amount to having the tip interacting with the water layer and the surface potentials as showed in figure 11. A sudden change in the tip sample distance causes the change of the interaction potential (this fact will be discussed later). From the graphs we can also distinguish the change in time response for different values of Q.

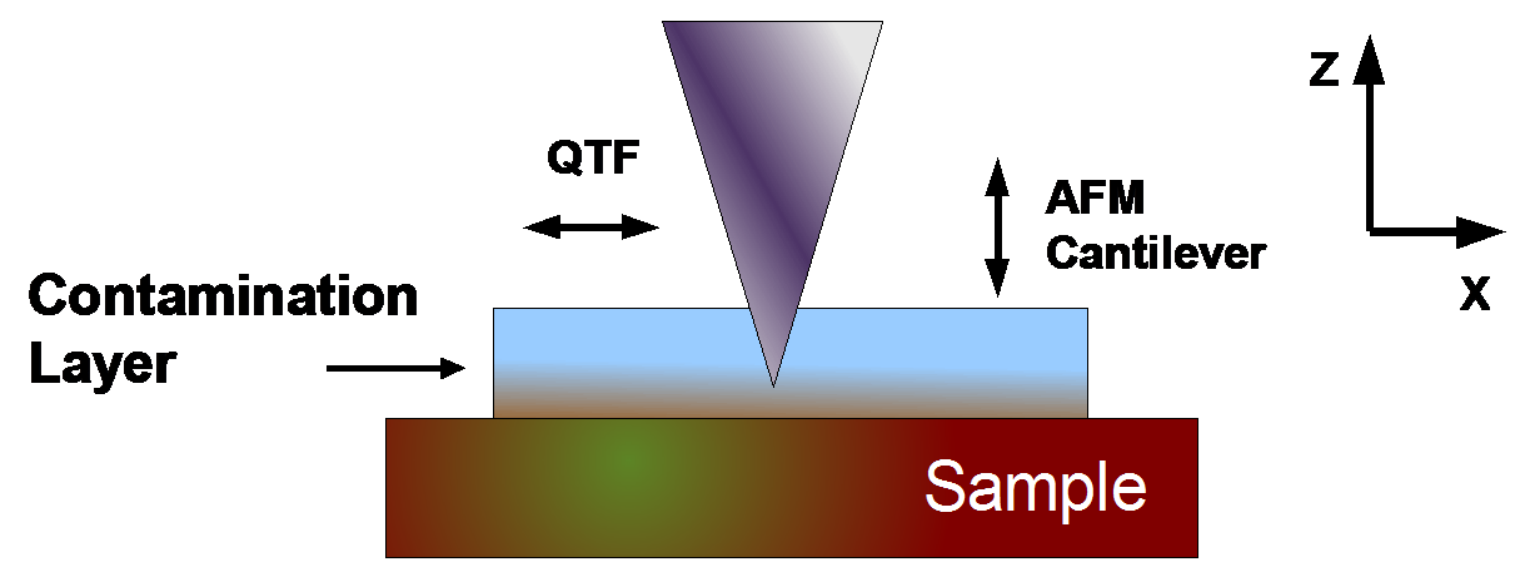

Figure 10. Difference between QTF and cantilever oscillation.

As a side note, the model presented has a slightly different interpretation for a QTF than for a AFM cantilever. Figure 10 shows that for a QTF with an oscillation parallel to the surface plane, the tip experiences a change in forces that are orthogonal to those experienced by a AFM cantilever. In the case of an AFM tip's motion the oscillation is parallel to the interacting potential variation in the $\mathrm{z}$ axis, as shown in figure 5, thus producing the force described in equation (2). In the case of a QTF, however, the tip is oscillating in a plane parallel to the surface, if aligned properly, and any interaction of the 
tip will have to originate from a lateral varying potential. Figure 12 shows the calculated transfer function for AM, $\|\bar{X}\|, \mathrm{PM}, \arg \bar{X}$, and RM, $\|\bar{X}\| \cos \theta$.

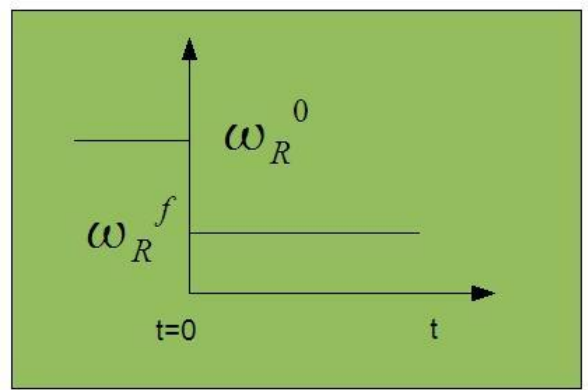

Numerical Simulation I

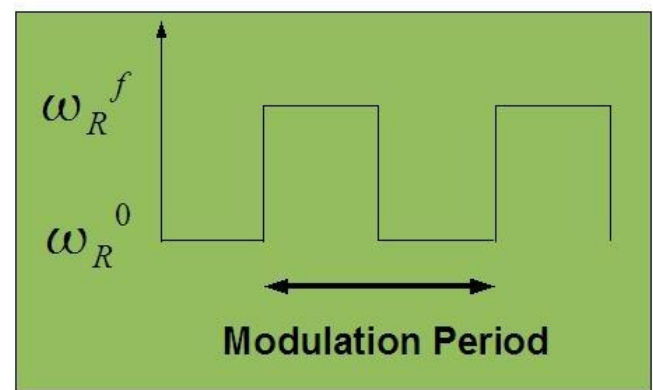

Numerical Simulation II

Figure 11. Calculation of the response of Amplitude, phase and $\mathbf{R}$ for a periodic interaction.

The bandwidth of the PM and RM are always greater than that of AM. AM has a cutoff frequency of about $40 \mathrm{~Hz}$ while PM and RM have a $100 \mathrm{~Hz}$ cutoff frequency.

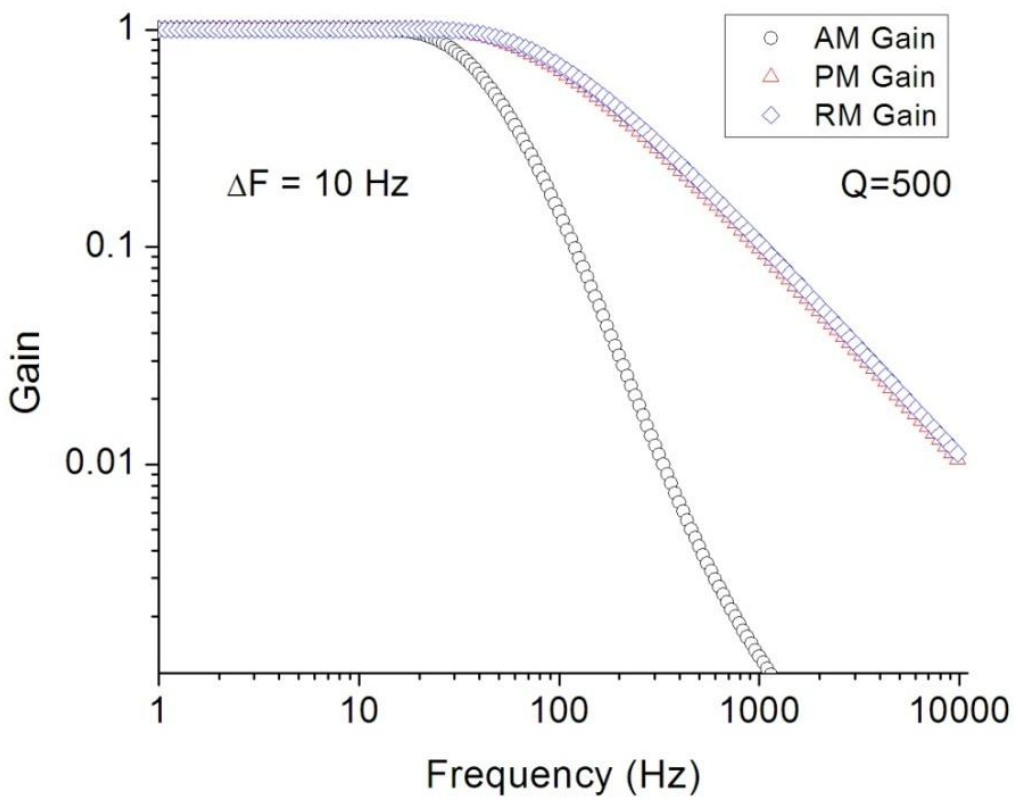


Figure 12. Transfer Function for AM, PM, and RM. The quality factor is 500 and the amplitude of the modulation is $10 \mathrm{~Hz}$.

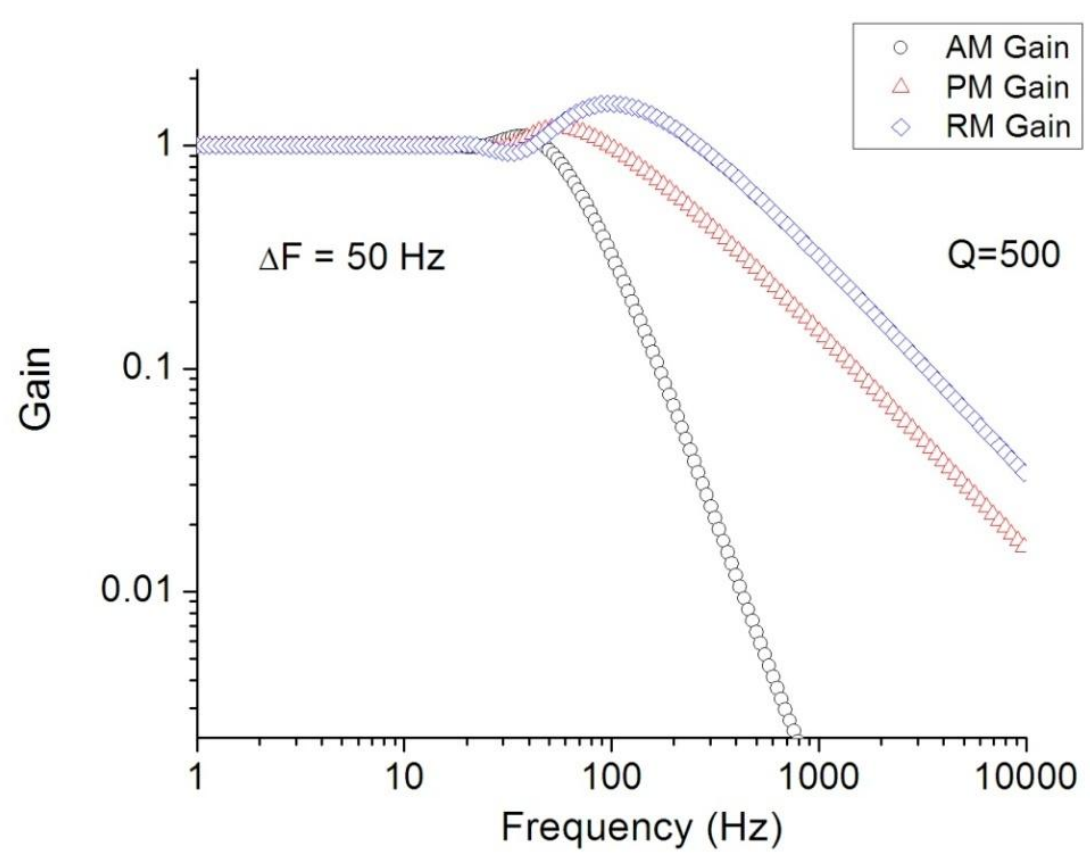

Figure 13. Transfer Function for AM, PM, and RM. The quality factor is 500 and the amplitude of the modulation is $50 \mathrm{~Hz}$.

Figure 13 shows same quality factor but a higher modulation amplitude. The $50 \mathrm{~Hz}$ change causes the RM to overshoot, but because of the relative small $Q$ the transfer functions are still stable. By increasing the quality factor we make the system more sensitive to any external influence but it also causes greater instability. Figure 14 shows how the AM bandwidth falls dramatically to about $10 \mathrm{~Hz}$ while RM and PM are still above $25 \mathrm{~Hz}$. RM has the larger bandwidth with $80 \mathrm{~Hz}$, but with larger overshoot.

A larger modulation bandwidth starts to create greater instabilities as it is showed in 
figure 15. It seems that the limits of the numerical model are being reached

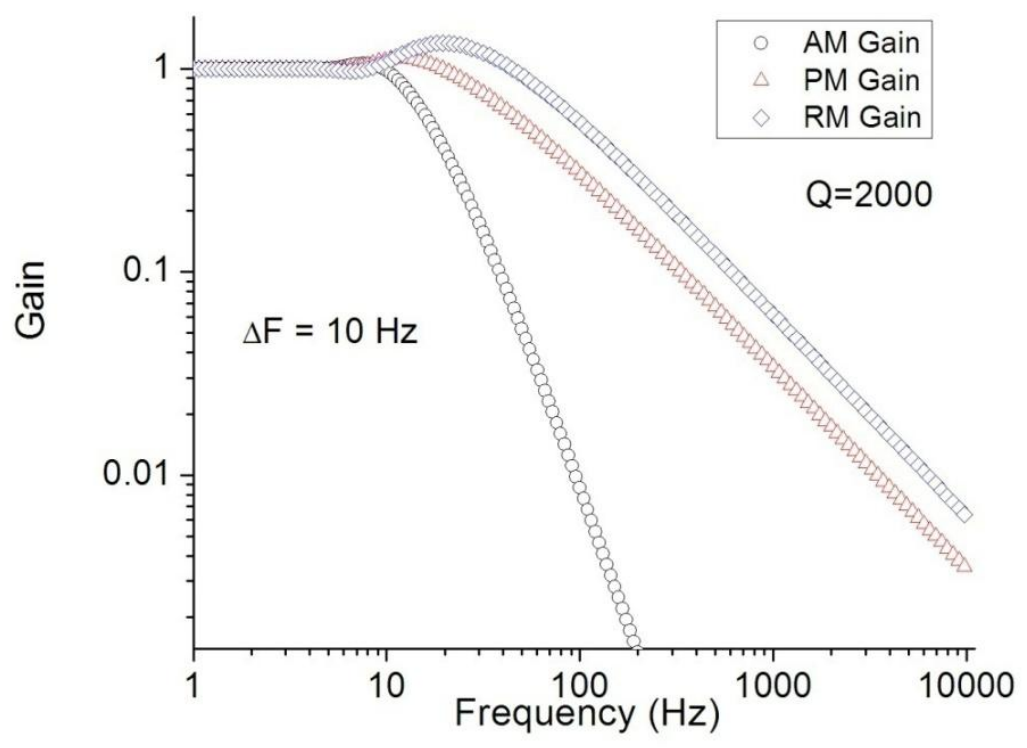

Figure 14. Transfer Function for AM, PM, and RM. The quality factor is 2000 and the amplitude of the modulation is $10 \mathrm{~Hz}$. .

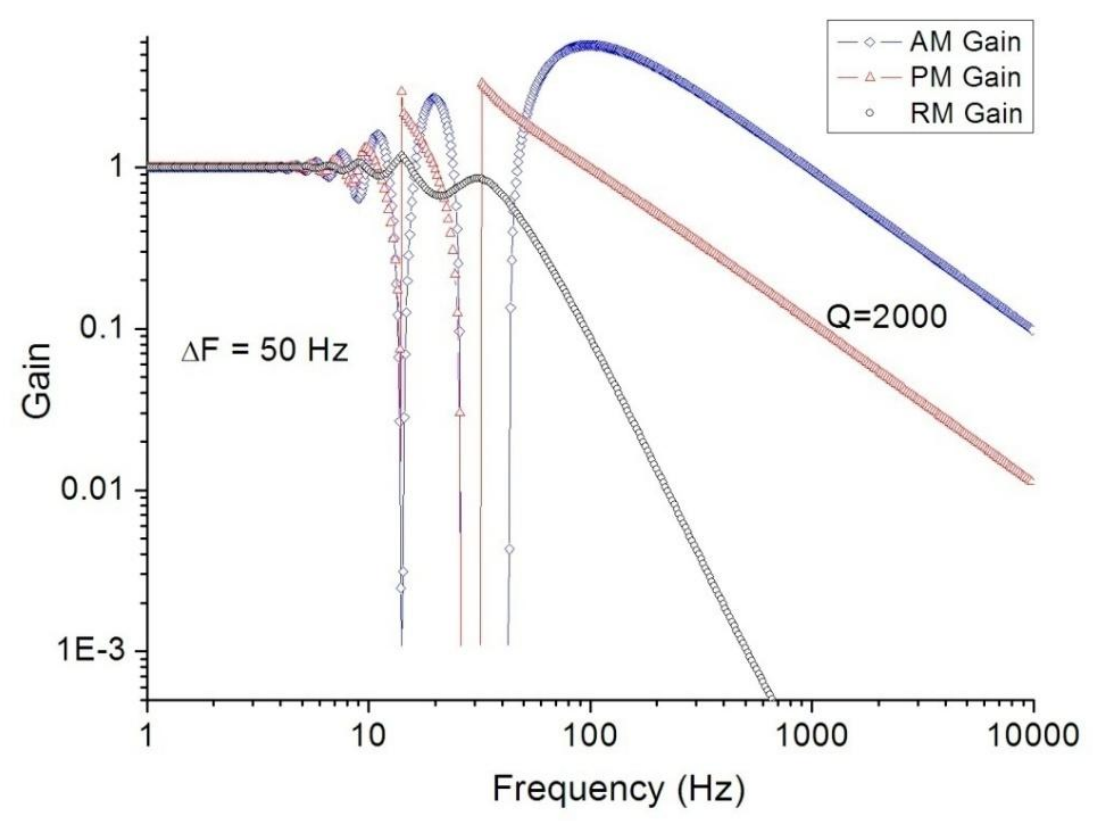

Figure 15. Transfer functions for $Q$ of 2000 and $50 \mathrm{~Hz}$ modulation amplitude. 
Finally, figure 16, shows that a higher Q decreases the amplitude of the modulation signal, given by $\Delta f$, at which instabilities start to appear. For a $\mathrm{Q}$ of 4000 and modulation amplitude of $20 \mathrm{~Hz}$ the system is already unstable.
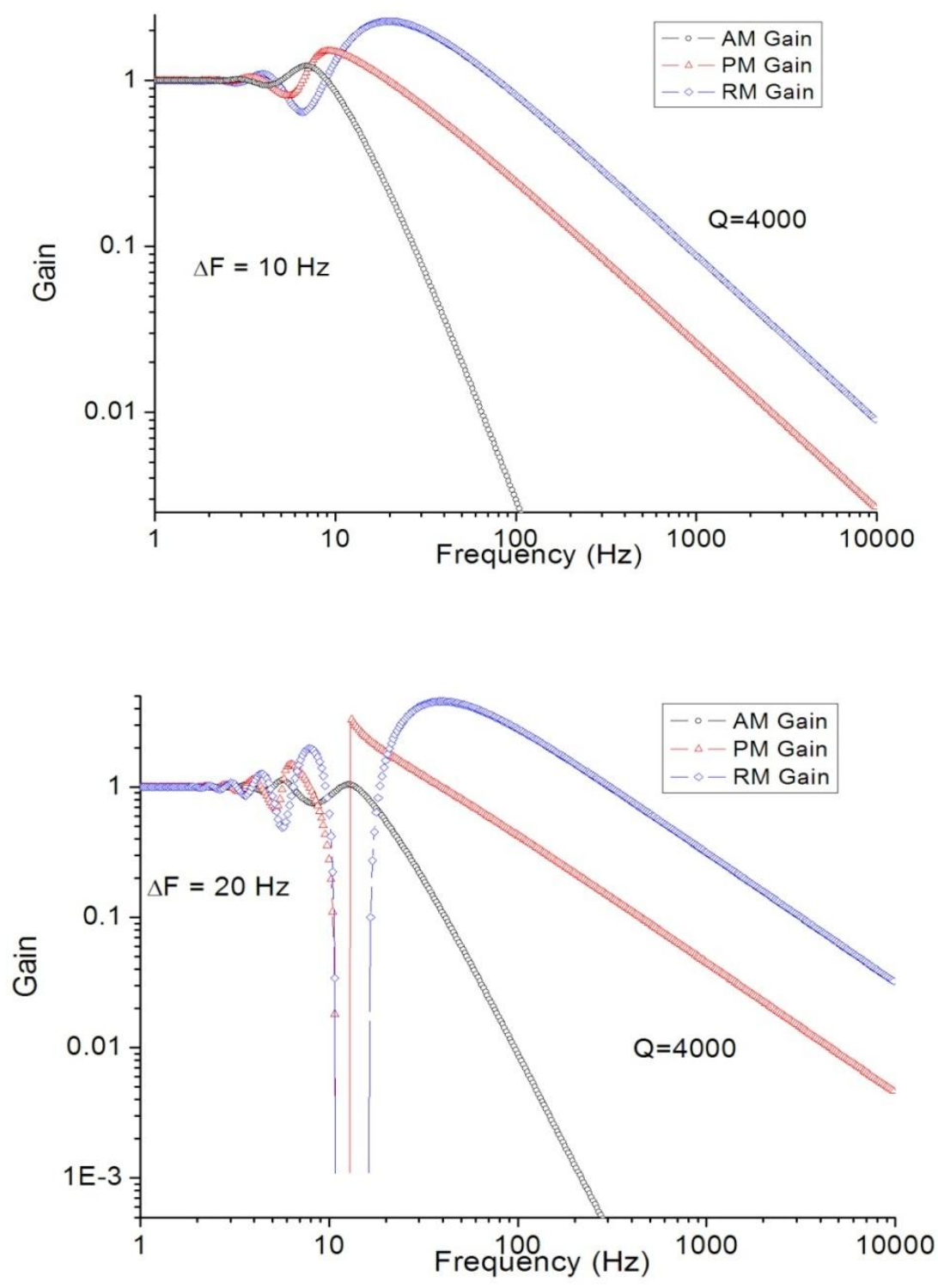

Figure 16. Transfer functions for $Q$ of $\mathbf{4 0 0 0}$ and high modulation amplitudes. 
In order to corroborate the results obtained with the numerical calculation based on the FHO model, a tip was brought close to the contamination layer and was carefully placed several nanometers above it. A sudden step motion towards the surface was performed and the amplitude, demodulated frequency shift and position of the piezo actuator were recorded. Figure 17 shows the obtained results. for the resonant frequency shift is proportional to the rate of change of the interaction force and not to the force itself, as shown in equation (5).

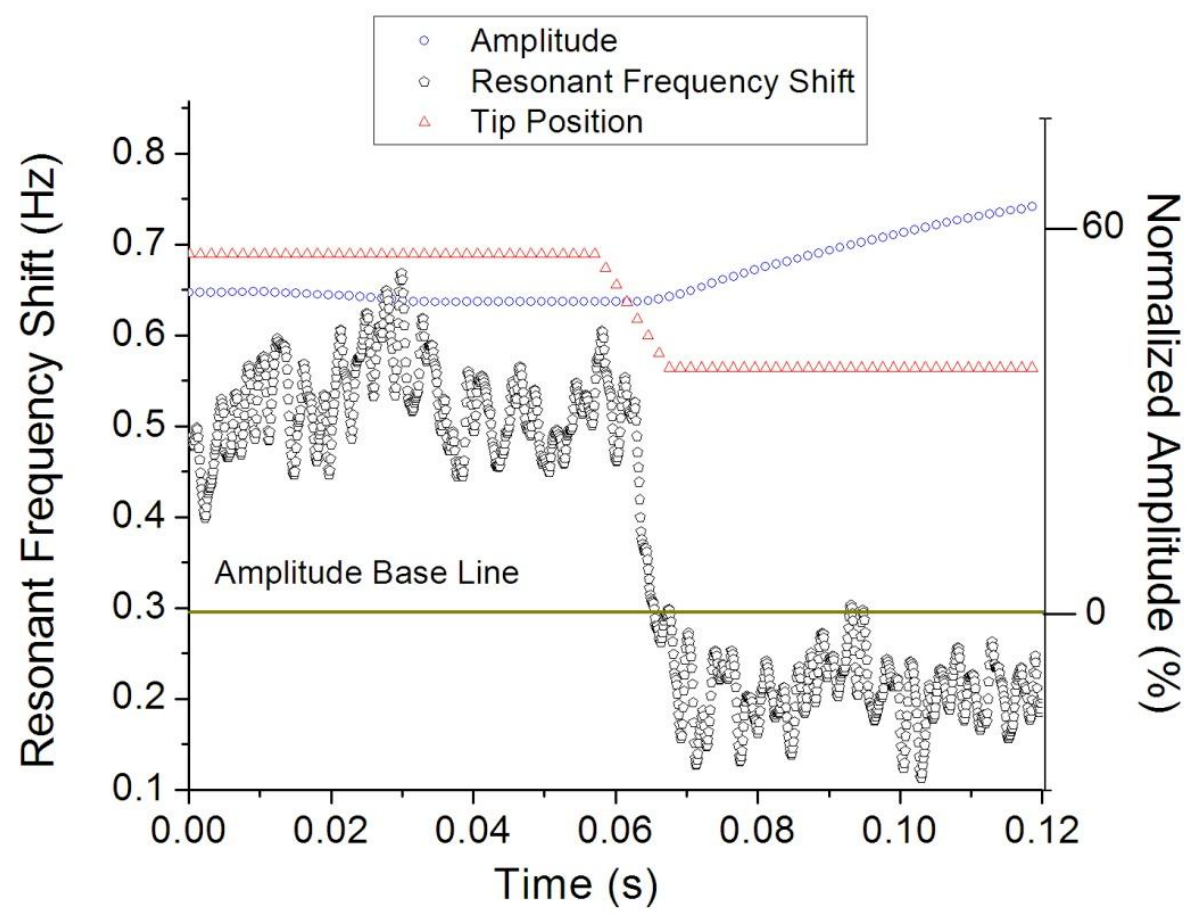

Figure 17. Experimental sudden change in resonant frequency. The frequency shifts to its final value $10 \mathrm{~ms}$ after the interaction.

The bandwidth of the PLL demodulation was $300 \mathrm{~Hz}$ (around $3.3 \mathrm{~ms}$ time constant $\tau$ ). After a time $2 \tau$ the final value is reached (63\% of the final value). 


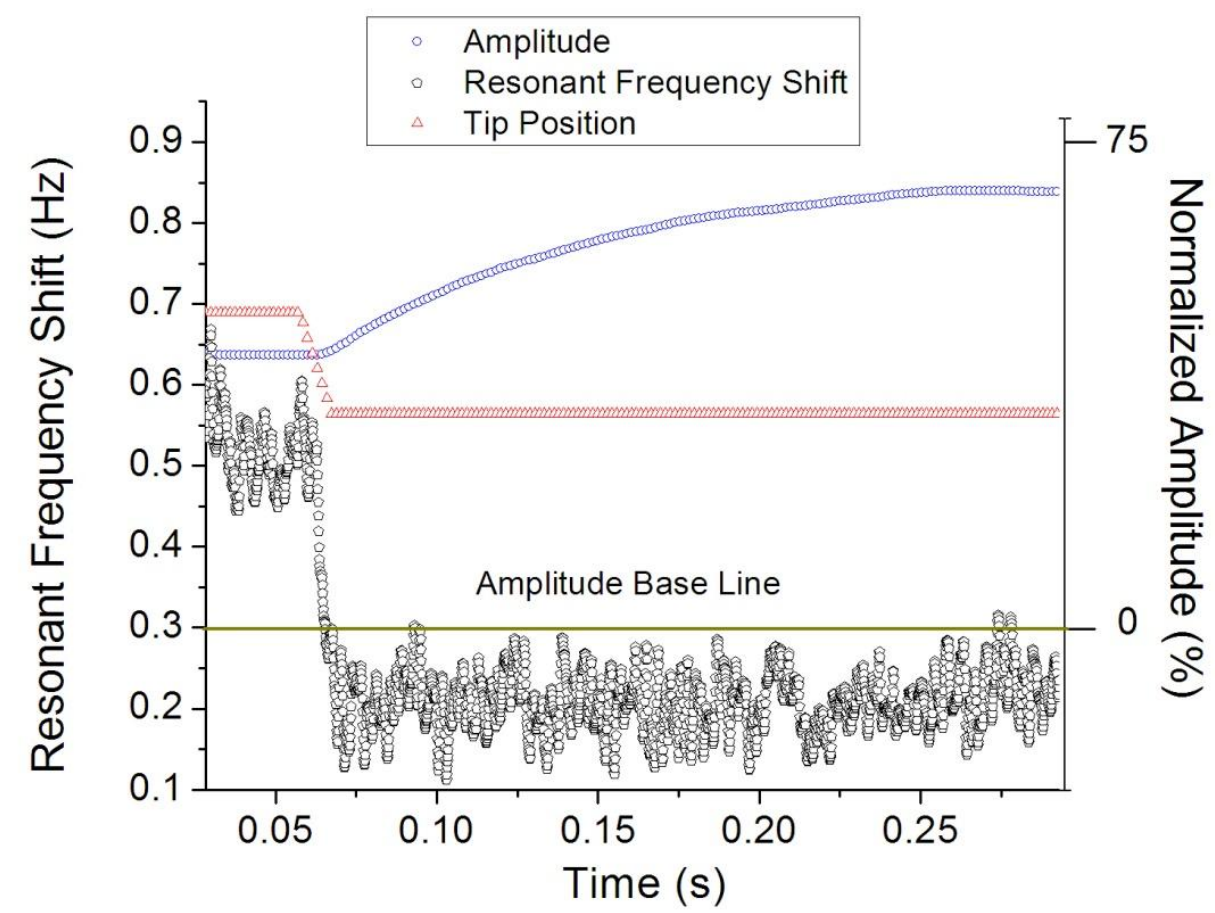

Figure 18. Experimental sudden change in resonant frequency. The amplitude changes to its final value $200 \mathrm{~ms}$ after the interaction.

Unlike the frequency shift, the amplitude measured with a time constant of $777 \mathrm{~Hz}$ (around $1.3 \mathrm{~ms}$ time constant) reaches its steady state after $85 \mathrm{~ms}$. This value corresponded very well with the experimental Q measured for this specific QTF, which gives a time response of $77 \mathrm{~ms}$ for $63 \%$ of the final value.

Finding an appropriate potential to describe the interactions can be done either experimentally or by DFT calculation. Given the ideal conditions the potential experimented by the QTF probe in the $\mathrm{z}$ direction should be constant, since the motion is only along the $\mathrm{x}$ axis. The varying potential appearing in equation 6 could be considered as arising from having the QTF making an angle with the surface normal, supplying the 
necessary component for the theoretical interacting force. An alternative source for this potential would be a surface with a changing potential along the $\mathrm{x}$ axis due to the atomic surface distribution. The atomic contribution to this change is usually too small compared to the tip size (a few hundreds of atoms in diameter). Yet another possible source for this potential is the contamination layer. Its contribution to the system is represented by the dampening force. It could be hypothesized that due to the small quantity of molecules being disturbed by the motion, the interaction of the tip with the water molecules could be represented by a local position dependent potential for certain oscillatory frequencies. It has been proposed [40] also that the stylus attached to the QTF and the QTF have a different phase in its oscillation. This motion can be modeled by adding a out of phase to the FHO model and provides a good description of some of the approach and retraction curves presented in the literature. The interactions are classified as even (conservative) and odd (non-conservative) interactions [48][49]. An additional tool for the characterization of the contamination layer would have to provide a technique to decouple both interactions. Such technique is described in the next section.

\subsection{Frequency Modulation Technique}

Every surface exposed to ambient conditions has an adsorbed layer of water molecules along with other elements present in air. This layer is commonly known as contamination layer or simply, water layer. It is formed due to the hydrophilic nature of the sample's surface as a result of the polarization produced by charging, the surface's molecular 
structure, or both. The layers' thickness reaches several nanometers and is also a function of the humidity level and its relative hydrophobicity. When interacting with the oscillating probe, the layer produces a dampening of the probe's oscillation. Being a dissipative force (non conservative), this process will not produce a resonant frequency shift of the QTF-probe system but an interaction with the surface potential will, as long as there is no formation of phonons acting as a dissipation channel [41]. The FM technique applied to SPM allows to decouple the conservative and non-conservative interactions by keeping the QTF excitation signal at its RF at all times. To achieve this it maintains the phase between the excitation voltage and the response of the QTF at 90 degrees by constantly changing the excitation signal frequency. If the QTF is always driven at its RF any decrease in the amplitude will be caused by a non-conservative interaction (viscosity of the contamination layer, friction from the surface). Hence, any decrease in the amplitude is only due to dissipative forces acting on the tip (figure 19). By keeping the excitation voltage constant we can calculate this dissipative force directly. In order to calculate quantitatively these interactions several theoretical techniques have been developed [48][49][50][51][52][53]. Sader [47] has found a relation that allows for the calculation of conservative interaction forces by knowing the frequency shift, and of dissipative forces by knowing the excitation force. In our setup, the excitation force can be monitored via the USTS. 


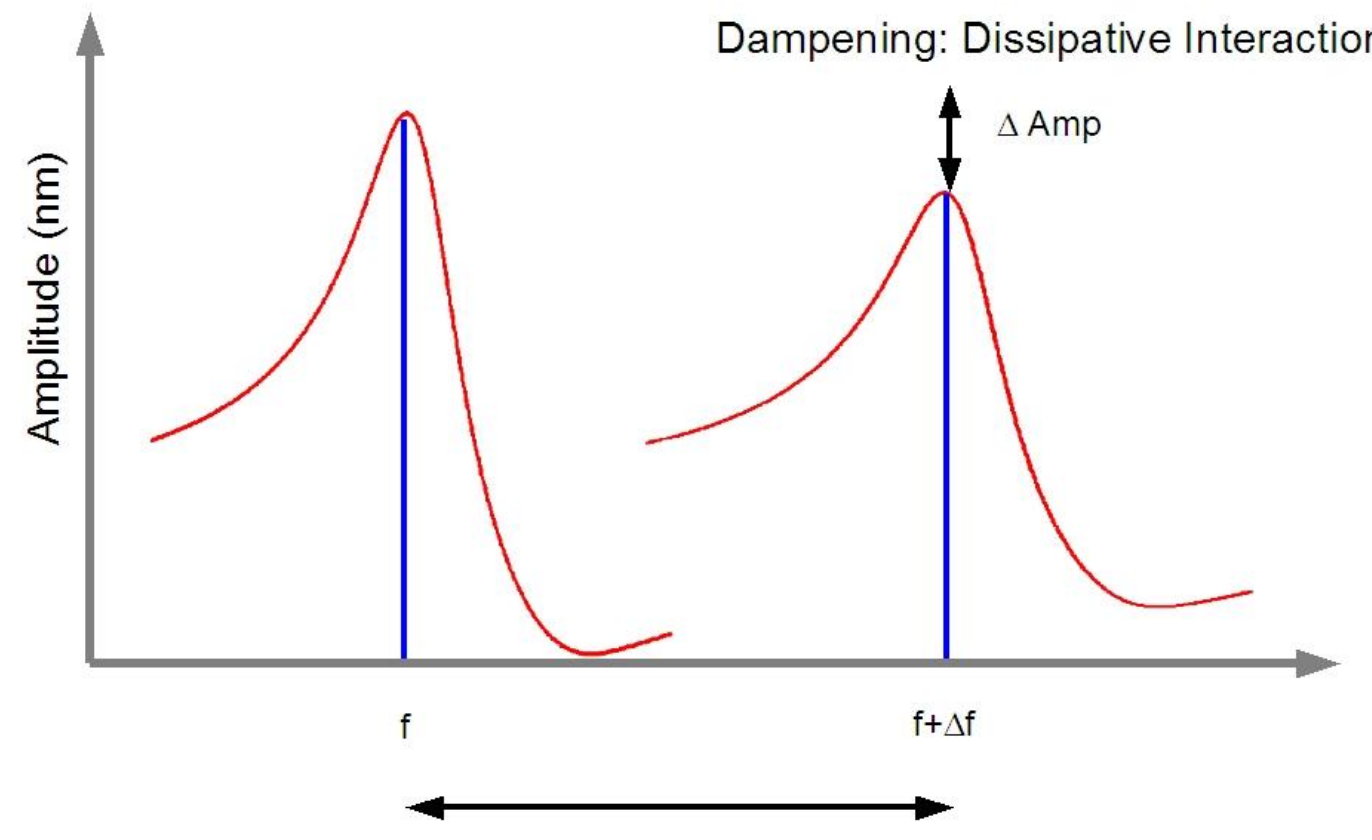

Frequency Shift : Conservative Interaction

Figure 19. Interpretation for the shift of the resonance frequency and the amplitude decrease in the TF spectrum.

There are discrepancies as to where conservative and dissipative forces begin in the course of approach and retraction curves and the nature of the interactions at distances higher than $8-10 \mathrm{~nm}$. The general agreement is that the formation of the meniscus and the long range interaction forces are responsible for interaction ranges above $10 \mathrm{~nm}$. Unlike $\mathrm{AM}$, the FM technique features a time response that is limited only by the time the sound waves travel through the whole QTF, determined by the sound velocity. This provides a theoretical limit to the detection of a frequency step. The heart of the FM technique is the 
PLL circuit which will be described next.

\subsubsection{PLL}

The first PLL's were implemented by Bellescize, a French engineer, in 1932, but only started being constructed with IC's around 1965. A PLL is an electronic circuit which in its locked state synchronizes a signal generated by an oscillator to a reference signal in both phase and frequency. In general, the individual components of the PLL are the phase detector, the low pass filter and the VCO (or a DCO in digital PLLs), all of them forming a closed loop circuit.
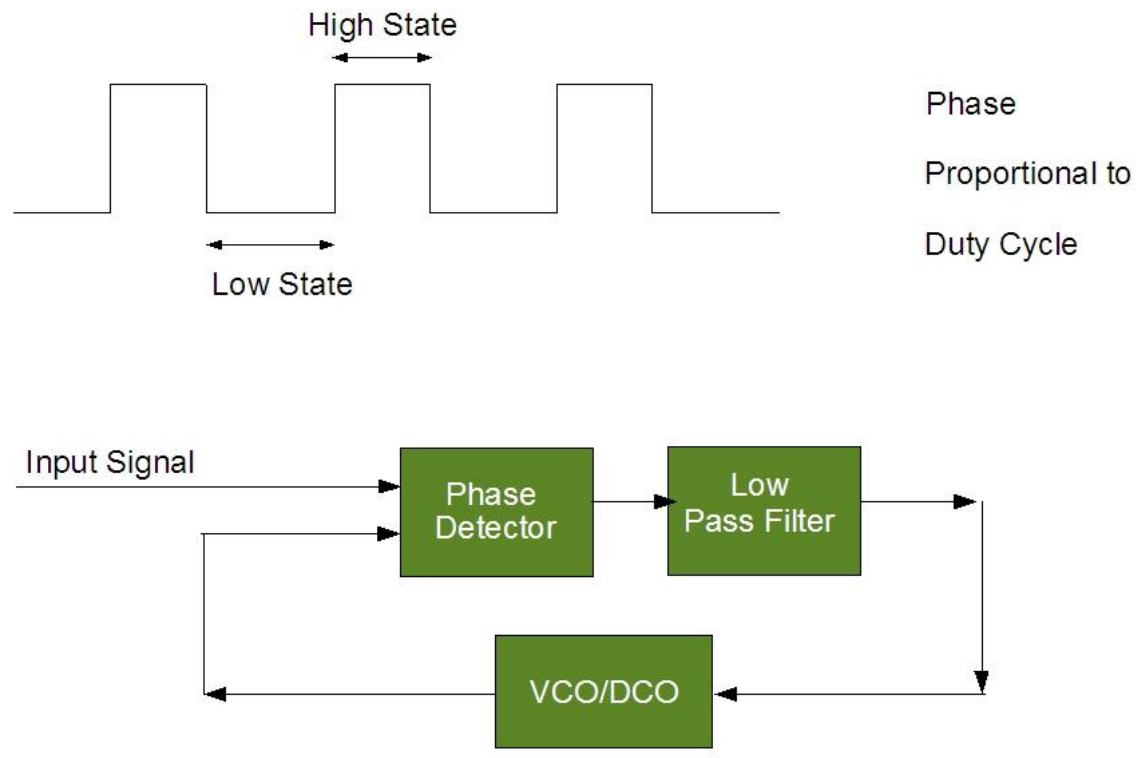

Figure 20. Phase Lock Loop diagram. 
The phase detector calculates the phase difference (in the case of the digital filter, defined as the zero level crossing of the square wave from the VCO/DCO) between the input, or reference signal, and the output signal of the VCO/DCO output. The output of the digital phase detector is a PWM signal whose DC component, recovered after a digital low pass filter stage, is proportional to the phase difference (or phase error) between the reference and the VCO/DCO output. This process is shown in figure 20.

A phase detector will measure phase changes relative to a setpoint. For any given wave signal the instantaneous frequency is defined as the change of phase with respect to time, $\frac{\partial \phi}{\partial t}$. Two signals with different frequencies will also have changing phase differences. Hence the value of $\frac{\partial \phi}{\partial t}$ will be different than zero and the output of the phase detector will start to move once a reference signal turned on. The value of the VCO output will stop changing when the phase difference is constant. This state of affairs is called the locked state and while in it the output of the LP is proportional to both phase (filtered output of the phase detector) and frequency shift (signal proportional to the output of the DCO). As a result, and depending on the characteristics of the PID control, either phase or frequency shift information can be used as the control signal. The main advantage of digital PLLs reside in their ability to keep a low noise level and to avoid the thermal drifting characteristic of analog VCOs. Faster signal processing is another advantage to be exploited. However, high frequencies are generally required to perform the detection since the circuit needs to have a clock cycle several times larger than its operating frequency. Analog counterparts do not usually have the same limitation. All Digital PLLs where an improvement, in terms of signal drift, with respect to previous analog based 
phase detectors. An intermediate step, the DPLL, is not entirely a digital device but a hybrid, such as the one included in the SR850 from Stanford Research, that has a digital frequency generator (which greatly enhanced the lock-in performance) together with an analog based phase detection and filtering. Unlike DPLL, ADPLL are entirely built from digital blocks and carries all of the advantages of digital signal manipulation. ADPLLs have been known to provide a faster lock in time and larger lock ranges. The OC-4 has a $1 \mu \mathrm{s}$ lower limit for the time constant. In practice, however, noise considerations at room temperature cause the time constant to be between $150 \mu$ s and $1 \mathrm{~ms}$.

\subsection{Quartz Tuning Fork Theory}

Due to its high stability, precision and low power consumption, the quartz crystal tuning fork has become a valuable basic component for frequency measurements. The behavior of the QTF is modeled as a RLC circuit using the electrical Butterworth-Van Dyke model [25] [26]. Being a piezoelectric, a variable amount of energy will expand and contract the QTF.

More specifically, there is a linear relationship between the amount of charge and the corresponding deflection of the QTF. The charge separation Q on the electrodes of the piezo material per mechanical deflection can be obtained experimentally by measuring the electromechanical coupling constant $\alpha$ [29], that has units of $\mathrm{C} / \mathrm{m}$. 

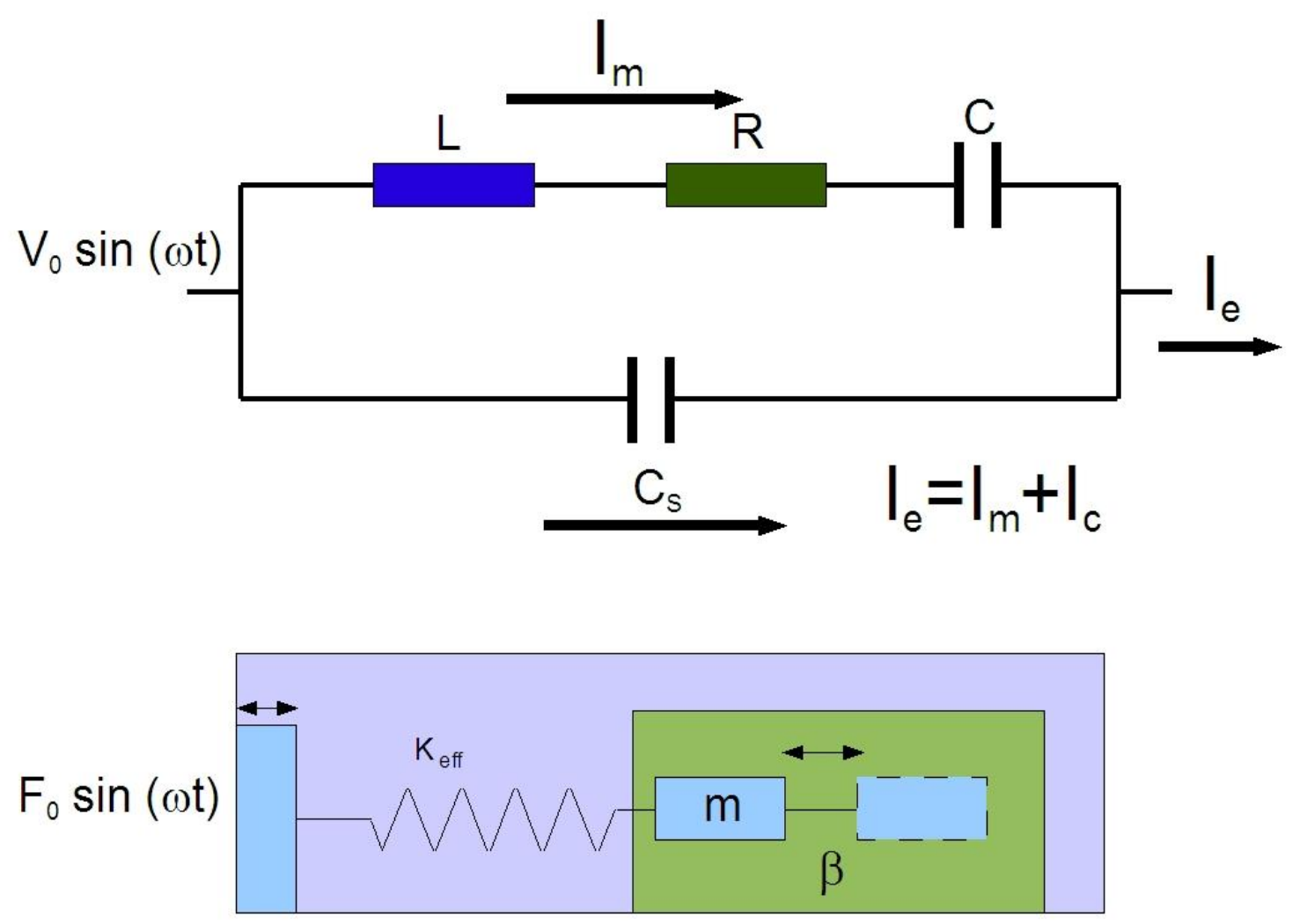

Figure 21. RCL circuit equivalent to the mechanical Butterworth-K. S. Van Dyke QTF model.

The electrical response and the mechanical deflection (measured by interferometry) of the QTF obtained simultaneously will yield $\alpha$ 's value using

$$
q=\alpha \Delta x
$$

where $q$ is in Coulombs and $\Delta x$ is in meters. However, if the equivalent elastic constant of the system is known (or modeled) it is possible to obtain $\alpha$ through the QTF equivalent circuit equations. Energy considerations will lead to the derivation of a relation between $K$ and $\alpha$. If we equate the potential energy produced by the QTF capacitance to that of 
the harmonic oscillator the relation

$$
\frac{q^{2}}{2 C}=\frac{K x^{2}}{2},
$$

is obtained, where $\mathrm{C}$ is Farads. Knowing that $q=\alpha \Delta x$ and after some algebraic manipulation the coupling parameter can be expressed by,

$$
\alpha=\sqrt{\frac{K C}{2}} .
$$

In his 1995 SPIE paper Karrai [37] derived the following equation for the determination of the elastic constant of one of the tines

$$
K=\frac{E}{4}\left(\frac{t}{L}\right)^{3} w,
$$

where $E$ is the Young's modulus of the QTF, $t$ is the thickness and $w$ is the width of the tine. In this fashion is possible to calculate $\alpha$ by knowing the dimensions and the material's properties. Furthermore, a relation between the RMS current and the displacement could also be derived. Starting with $I=\alpha \dot{x}$, it can be shown that

$$
\Delta I_{R M S}=4 \pi f \alpha\left(\Delta x_{R M S}\right),
$$

which produces the RMS oscillation amplitude $\Delta x_{R M S}$ for the QTF. As observed in figure 21 the measured current is the result of two contributions: the current through the QTF, 
$I_{p}$, and a parasitic signal, $I_{c}$, which is proportional to the frequency and is in phase with the excitation signal. When the excitation voltage is approaching the resonant frequency, the magnitude of $I_{c}$ remains the same but the phase of $I_{p}$ changes (figure 23), making the final curve to be asymmetric as shown in figure 23. Several methods to address this problem have been proposed [59][60][61]. Solving the RLC circuit for the total current $I_{e}$ [59] shown in figure 21 and calculating the amplitude $A_{e}$ and the phase $\theta_{e}$

$$
\begin{gathered}
=\frac{I_{0} \omega}{Q \omega_{0}} \sqrt{\left.\frac{1+2\left(C_{0} / C\right)\left(1-\omega^{2} / \omega_{0}^{2}\right)+\left(C_{0} / C\right)^{2}\left(1-\omega^{2} / \omega_{0}^{2}\right)^{2}+\left(C_{0} / C\right)^{2}\left(\omega /\left(\omega_{0}\right.\right.}{\left(1-\omega^{2} / \omega_{0}^{2}\right)^{2}+\left(\omega /\left(\omega_{0} Q\right)\right)^{2}}\right)} \\
\quad \theta=\arg \left(\frac{\omega}{\omega_{0} Q}+i\left(1-\frac{\omega^{2}}{\omega_{0}^{2}}+\frac{C_{0}}{C}\left(1-\frac{\omega^{2}}{\omega_{0}^{2}}\right)^{2}+\frac{C_{0}}{C}\left(\frac{\omega}{\omega_{0} Q}\right)^{2}\right)\right)
\end{gathered}
$$

The stray current $I_{s}$ is given by $\omega C_{0} V_{0} \sin (\omega t)$. If we calculate $I_{m}=I_{e}-I_{s}$ and obtain its amplitude and phase we get

$$
\begin{gathered}
A_{m}=\sqrt{A^{2}-2 \omega C_{0} V_{0} A \sin \theta+\left(\omega C_{0} V_{0}\right)^{2}}, \\
\theta_{m}=\arg \left(A \cos \theta+i\left(A \sin \theta-\omega C_{0} V_{0}\right)\right) .
\end{gathered}
$$

Fitting the SHO model equation to the measured spectrum we obtain the parameters $I_{0}, \omega_{0}, C_{0} / C$ and $Q . R$ is obtained from $R=V_{0} / I_{0}$ where $V_{0}$ is the excitation voltage amplitude. The values for $L, C$ and $C_{0}$ are calculated from the known equations $\omega_{0}=\frac{1}{L C}$ 
and $L=\frac{R Q}{\omega_{0}}$. For the calculation of $\alpha$ only $\mathrm{C}, \mathrm{K}$ and equation (12) are needed. Finding $\Delta X_{R M S}$ comes in a straightforward manner from equation 14.

Recently [38] proposed a three spring model to account for the oscillation of both the tines and the juncture of the QTF, producing an effective spring constant of $k_{e f f}=$ $2\left(k+2 k_{c}\right)$ where $k$ is the spring constant of one of the tines and $k_{c}$ is the spring constant of the juncture between the two prongs. This change however important is not crucial for the feedback mechanism since it represents a constant RF shift in equation 6. Its main impact would be on the calculation of the oscillation amplitude of the QTF tines.

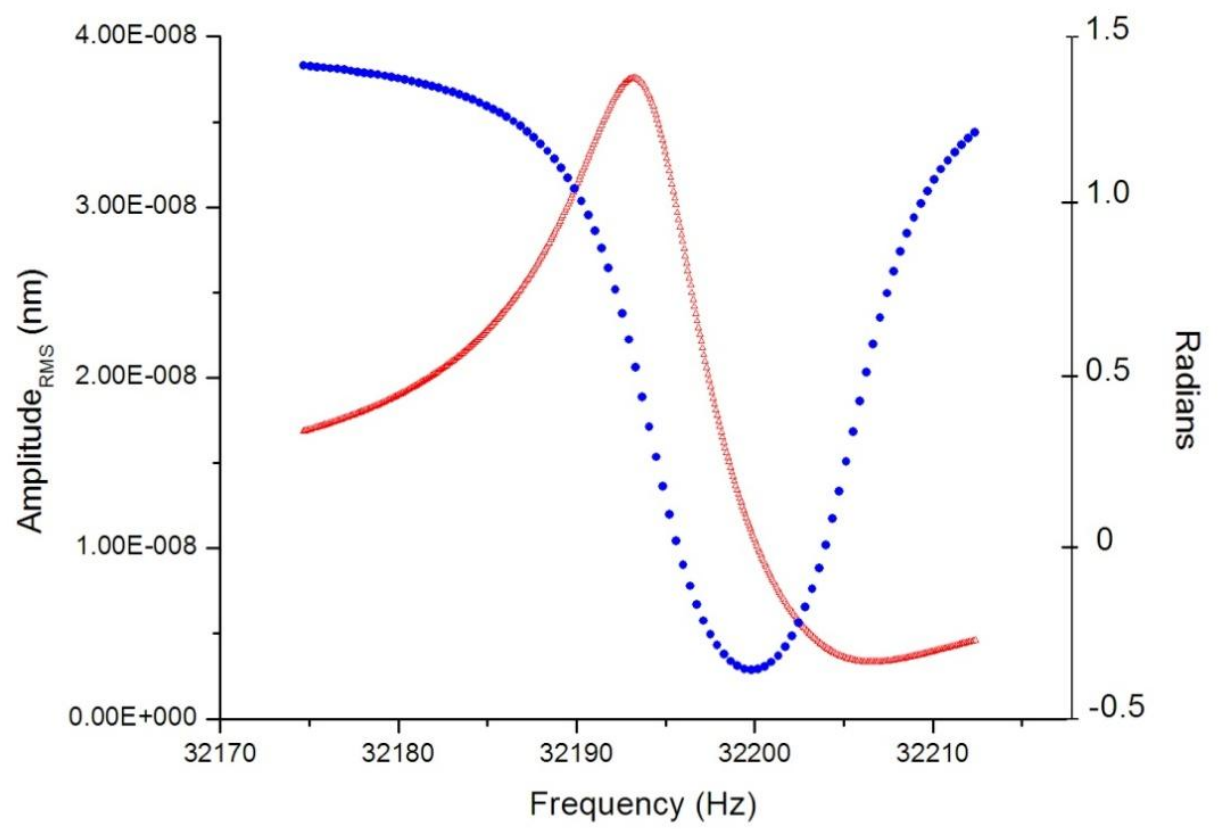

Figure 22. Raw QTF response signal. 


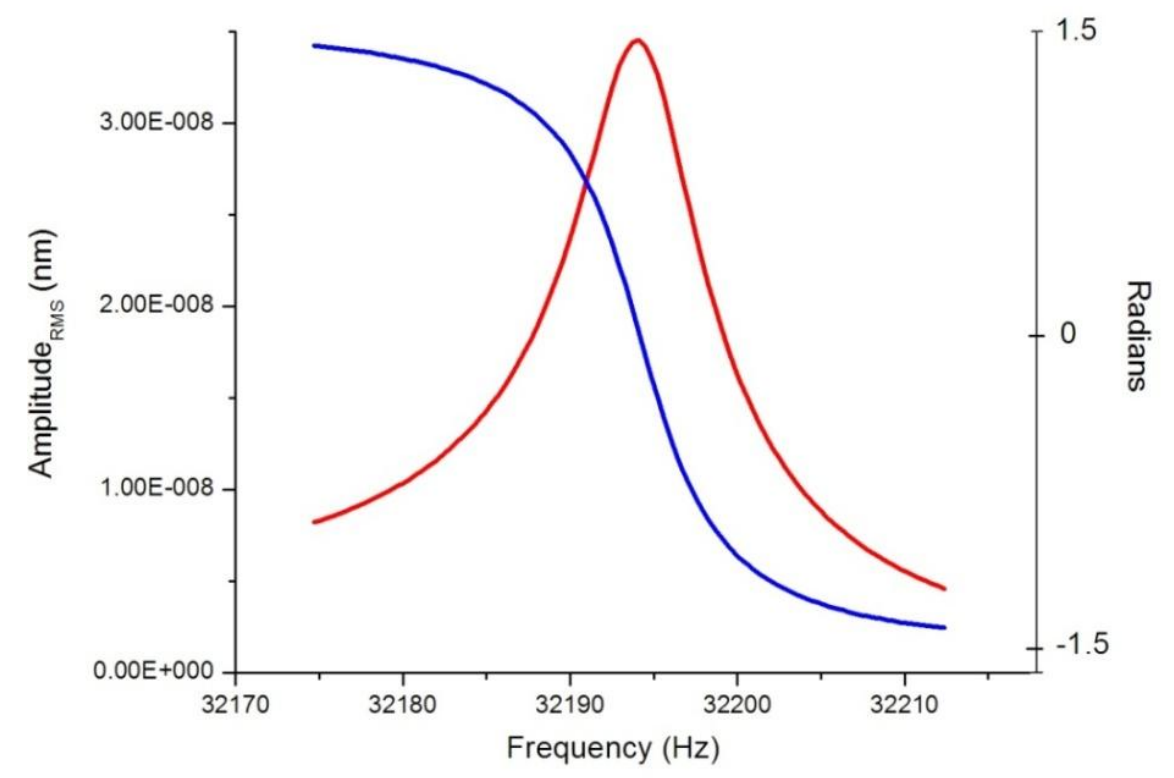

Figure 23. Processed QTF response signal.

It is time to introduce the quality factor concept, known simply as Q. It represents the ratio of the energy stored and the energy dissipated in one cycle of oscillation. Since the higher the excitation voltage the larger the oscillation's amplitude, we are always looking to minimize the energy given to the QTF without sacrificing the SNR. As a result a higher Q should be the better choice. Unfortunately, when using the amplitude modulation in SPM, the settling time of the QTF-probe system is directly proportional to the $\mathrm{Q}$ value, thus making the scanning speed slower with higher $\mathrm{Q}$. This type of competing parameters is common in physics and the best choice has to be made on a case by case basis. 


\subsection{Piezo Stages}

Piezo positioners are ubiquitous in SPM technology. They make possible the precise positioning of the probe, the scanning of the sample and the excitation at high frequencies. A simple molecular model would explain the piezoelectric effect as a polarization of the materials molecules due to the stress induced reaccommodating molecules. The inverse is also possible: electric polarization would result in structural change. Experimentally, the excitation signal driving the piezo is directly related to its motion. By differentiating equation (10) with respect to time, we obtain the following relations,

$$
\begin{gathered}
I=\alpha \dot{x} \\
\frac{d I}{d t}=\alpha \ddot{x}
\end{gathered}
$$

As circuit elements piezo stages are essentially capacitors that are charged and discharged. The amount of charge will determine the deflection, i.e. the displacement, experienced by either probe or sample. Piezo materials also have inherent hysteresis, creep, etc. corrected with closed loop feedback control. A paramount requirement in metrology is the accuracy of the position, thus making piezo positioner's closed loop implementation practically mandatory. As a result, the system contains a closed loop within the Feedback system, a closed loop within a closed loop. For this reason is 
necessary the inner loop to be faster than the response of the overall feedback positioning loop, thus preventing instabilities.

The next section will explain the feedback positioning mechanism.

\subsection{The Feedback Mechanism}

The feedback mechanism is a process that controls a system's dynamics in order to make it stable in time. In the particular case of SPM systems it controls the probe-sample distance using a piezo positioner. The basic configuration of a feedback contains the components previously described (QTF, PID, PLL, Piezo positioners). It reacts to the changes of the QTF signal in order to keep the interaction at a roughly constant level while the sample is being scanned. When the PLL is able to track the input signal, in a given frequency range, it is said to be 'in lock'. The ability of the feedback to maintain

the tip-sample distance is a measure of the stability of the feedback. A phase delay between the actual probe-sample interaction and the corresponding displacement reaction exists and will determine the maximum scanning velocity possible without losing the 'locked state' which would cause either the breaking of the probe or unwanted oscillations, resulting in poor image quality. Obtaining an expression for this delay is beneficial when in the building stage, and subsequently experimentally determined by measuring the feedback's response to a step function. It can be showed [11] that the closed loop delay is related to the open loop delay through a simple equation PID system. 
Open loop delay can be calculated by algebraically adding the delays of every individual part of the feedback loop. The derivation of such equation is presented here for completeness. The total feedback loop is presented in figure 24 .

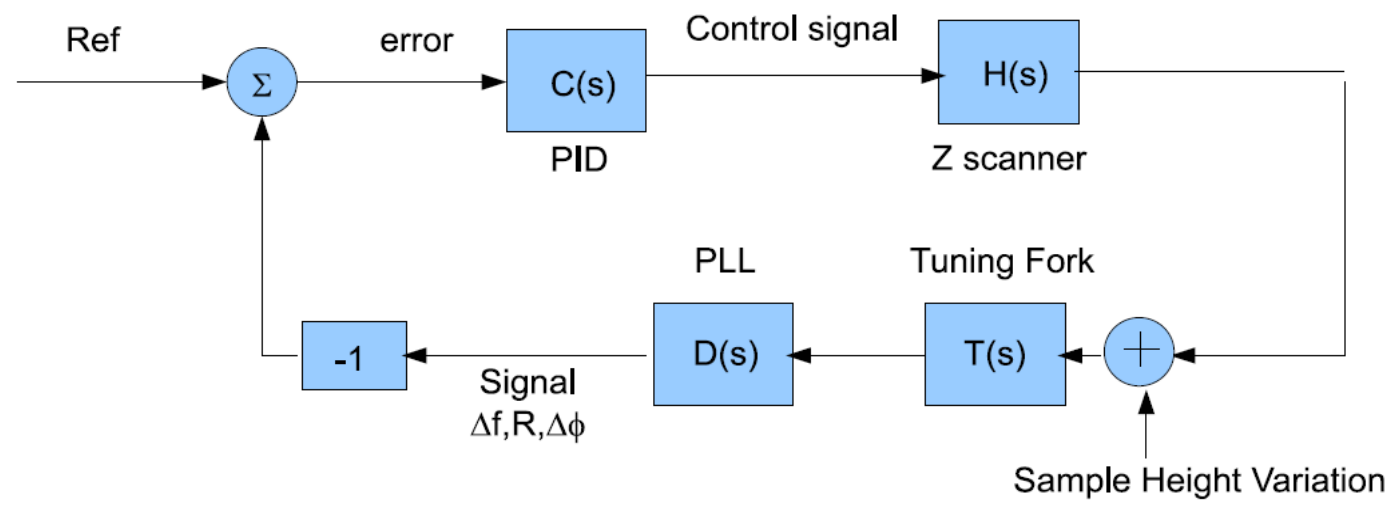

$\begin{array}{ll}T(s) \text { : Tuning Fork Transfer Function } & \text { C(s):PID Transfer Function } \\ H(s) \text { : Z Scanner Transfer Function } & \text { D(s):PLL Transfer Function }\end{array}$

Figure 24. Feedback loop transfer function of a general SPM.

The transfer function obtained from figure 24 is,

$$
K(s)=\frac{-H(s) C(s) T(s) D(s)}{1+H(s) C(s) T(s) D(s)}
$$

Without knowing the actual functions $H(s), C(s), T(s)$ and $T(s)$, is possible to estimate the phase difference between closed loop and open loop. If we make $A(s)=$ $H(s) C(s) T(s) D(s)$ an then we can write, 


$$
K(s)=\frac{-A(s)}{1+A(s)}
$$

with,

$$
A(i \omega)=A_{0}(i \omega) e^{\omega}
$$

It is then straightforward to obtain the full transfer function in terms of $A(s)$,

$$
\begin{gathered}
K(i \omega)=\frac{-A_{0} \cos \theta(\omega)-i A_{0} \sin \theta(\omega)}{1+A_{0} \cos \theta(\omega)-i A_{0} \sin \theta(\omega)} \\
K(i \omega)=\frac{-A_{0}^{2}-A_{0} \cos \theta(\omega)+i A_{0} \sin \theta(\omega)}{1+2 A_{0} \cos \theta(\omega)+A_{0}^{2}}
\end{gathered}
$$

So far we have only shaped (21) into a simpler form. The gain and phase delay are,

$$
\begin{gathered}
K(i \omega)=\frac{A_{0}}{\sqrt{1+2 A_{0} \cos \theta(\omega)+A_{0}^{2}}} \\
\tan \Phi(\omega)=\frac{-\sin \phi(\omega)}{A_{0}(\omega)+\cos \phi(\omega)}
\end{gathered}
$$

respectively. If the gain is maintained at $|K(i \omega)| \approx 1$ a value for $T_{0}(\omega)$ can be obtained, 


$$
A_{0}(\omega)=\frac{-1)}{2 \cos \phi(\omega)}
$$

and if we substitute 28 into 27 we obtain,

$$
\Phi(\omega)=\pi-2 \phi(\omega)
$$

Since the $\pi$ constant is caused by the direction of $\mathrm{Z}$ scanner displacement, equation (15) shows the phase delay of the closed loop is twice the phase delay of the open loop, provided the gain is maintained at $\sim 1$.

To calculate the open loop total delay we need only to add the transient response of every component. The previous result makes possible to estimate the closed loop delay by knowing the individual delays of the components. When modifications or improvements are made, the ability to estimate this delay is crucial in the choosing the appropriate scanning speed because it depends on how fast the feedback will react to sudden changes in the interactions. The delay introduced by the WGAS feedback is equal to the time response of the USTS sensor transducer plus the time it takes the sound to travel from the QTF to the resonant cavity surface. The time response of the USTS is in the order of a few microseconds at the most.

Frequency Modulation relies on having the phase constant (at zero value), therefore any attempt of phase change will be translated into a change into a response from the PLL frequency to maintain it constant. It follows then that FM is slower than PM because of the delay introduced by the $\mathrm{VCO} / \mathrm{DCO}$. Its advantage consists in that any decrease in the oscillation amplitude will be caused by a dissipative process and not a conservative one, 
allowing the FM feedback control to decouple both interactions. In PM feedback control mode this is not possible and will require a recalibration of the driving frequency for different tip-sample distances. The sound takes approximately $100 \mu$ seconds to reach the sensor, with the present geometry. Further design improvements must have this parameter in consideration. 


\section{Chapter 3}

\section{Experimental Details}

In implementing the WGAS feedback several tasks were overtaken. PID control, scanning and approach and retraction curves capabilities through FPGA programming, stage conditioning for acoustic measurements on the WG, probe preparation, piezo stages characterization, signal conditioning, and QTF parameters determination. The following sections will give a description of each one of these items. The experimental final setup of the WGAS feedback, and the optional USBS, is presented in figure 25. Both the sensor used for the feedback, USTS, and the one used for sample characterization, USBS are shown. The spectrum of the sensor has a sensitivity of about $1 \mathrm{~nm}$ per Volt [62][63][46] for both sensors. They are off-the-shelve Acoustic sensors from DECI. 


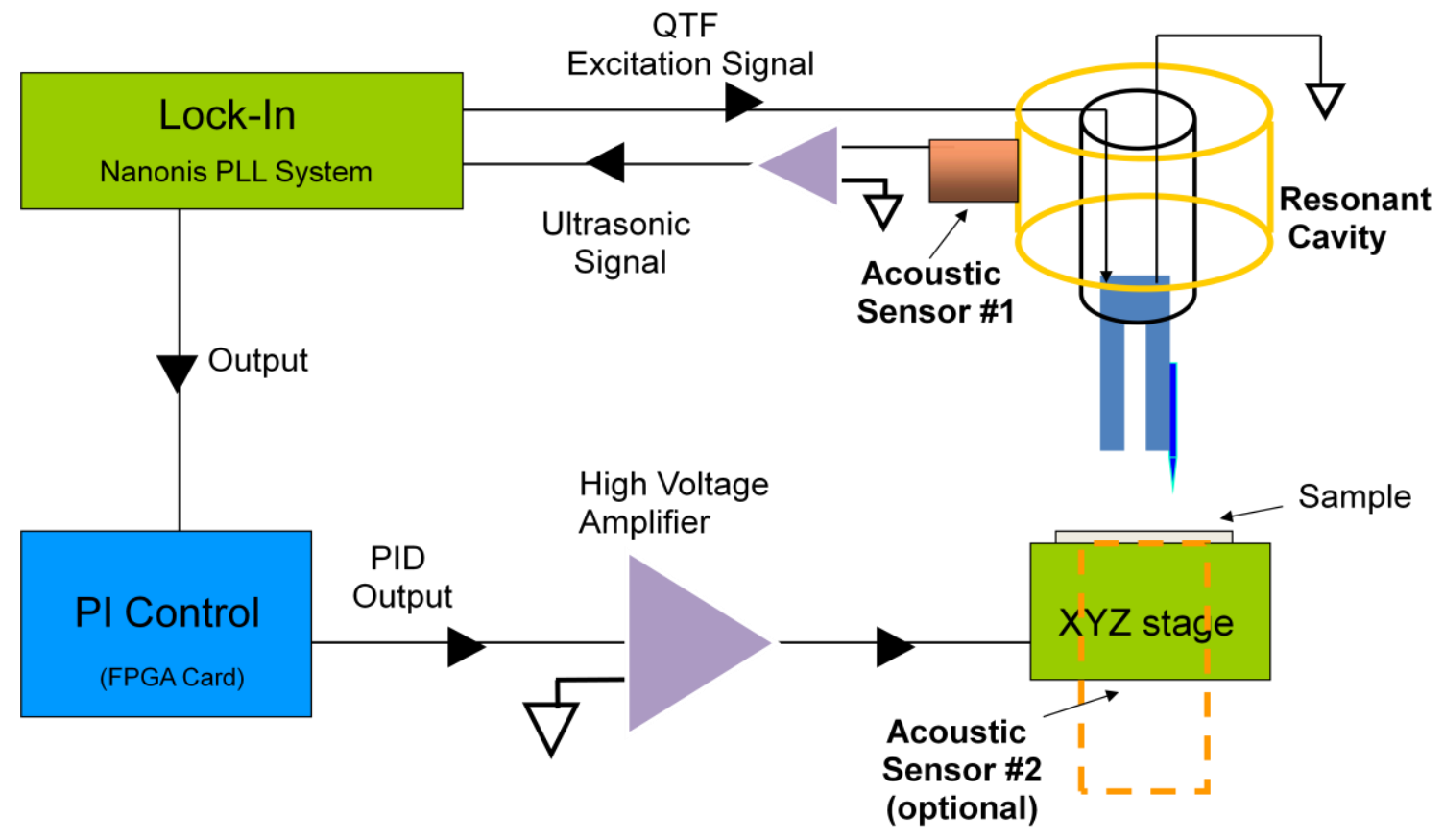

Figure 25. Experimental Setup Diagram.

\subsection{Quartz Tuning Fork Parameters}

The QTF parameters presented in section 5 were calculated by fitting the experimental spectrum. The QTF has a $\mathrm{Q} \approx 10000$ in air. This value reduces to $6000-9000$ when a coated/uncoated fiber tip is mounted on one of the prongs, and to a value between 10004000 for a gold tip. An additional mass mounted on the remaining prong will increase the Q value due to the restored symmetry [64]. The QTF is shown in figure 26. A mounted fiber tip is shown in 26 . The spectral response and the fitted curve (continuous line) is shown in figure 27. To make this possible a model for the elastic constant K of the QTF had to be included, as introduced in section 5. A model for the calculation of the elastic 
constant $\mathbf{K}$ was proposed by [38].

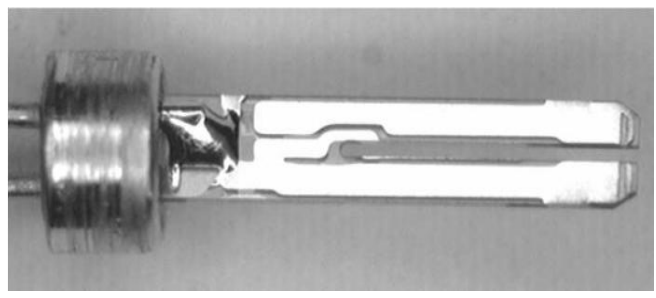

Quartz Tuning

Fork without the

metal encase

$Q \approx 10000$

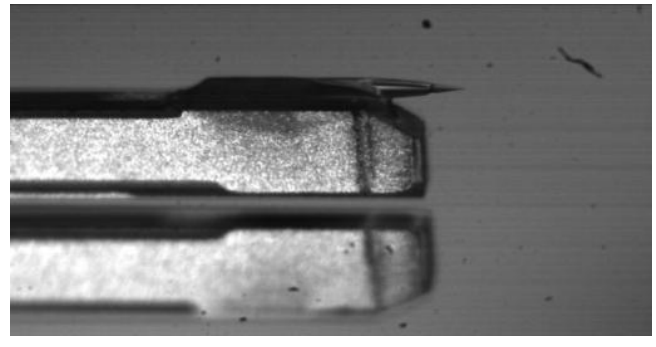

$Q \approx 6000$
Quartz Tuning Fork without the metal encase and a mounted fiber probe

Figure 26. Tuning fork outside its enclosure, exposed to air. Fiber tip mounted in one of the tines.

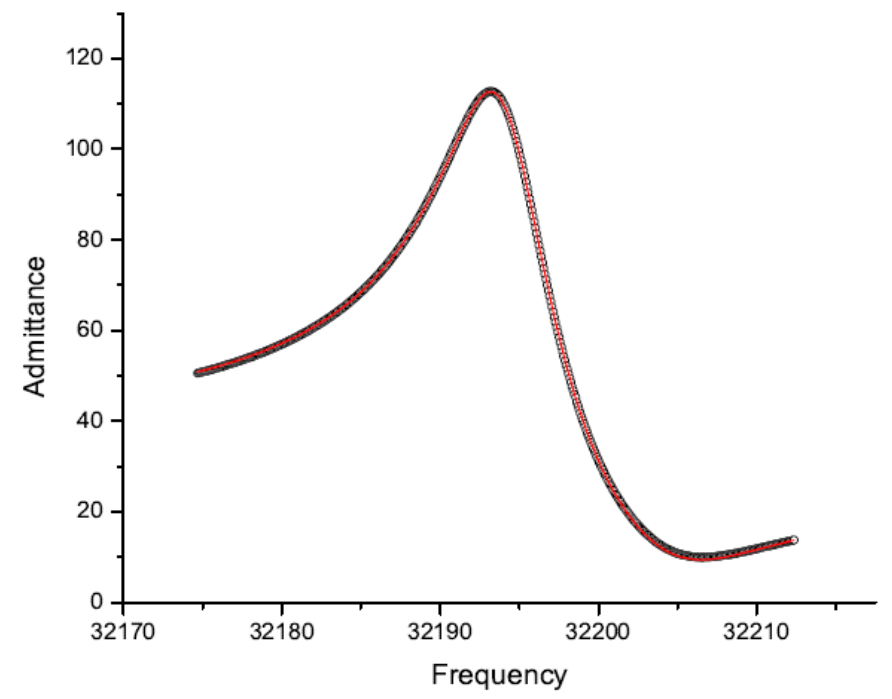

Figure 27. Example of fitted QTF raw data.

The calculated parameters are shown in table 1 . Using $\mathrm{K}$ from the chosen elastic model and $\mathrm{C}$ from the fitting procedure of the QTF the coupling piezoelectric constant $\boldsymbol{\alpha}$ is 
obtained along with the amplitude of oscillation with equation (14).

Table 1. Tuning Fork parameters for gold and fiber tips

\begin{tabular}{|l|l|l|l|}
\hline TF tip & $\mathrm{K}(\mathrm{N} / \mathrm{m})$ & $\mathrm{C}(\mathrm{F})$ & $\square(\mathrm{C}$ \\
\hline Fiber tip & 26300 & $2.02 \times 10^{-15}$ & $5,15 \times 10^{-6}$ \\
\hline Gold tip & 26300 & $0.49 \times 10^{-15}$ & $2,55 \times 10^{-6}$ \\
\hline
\end{tabular}

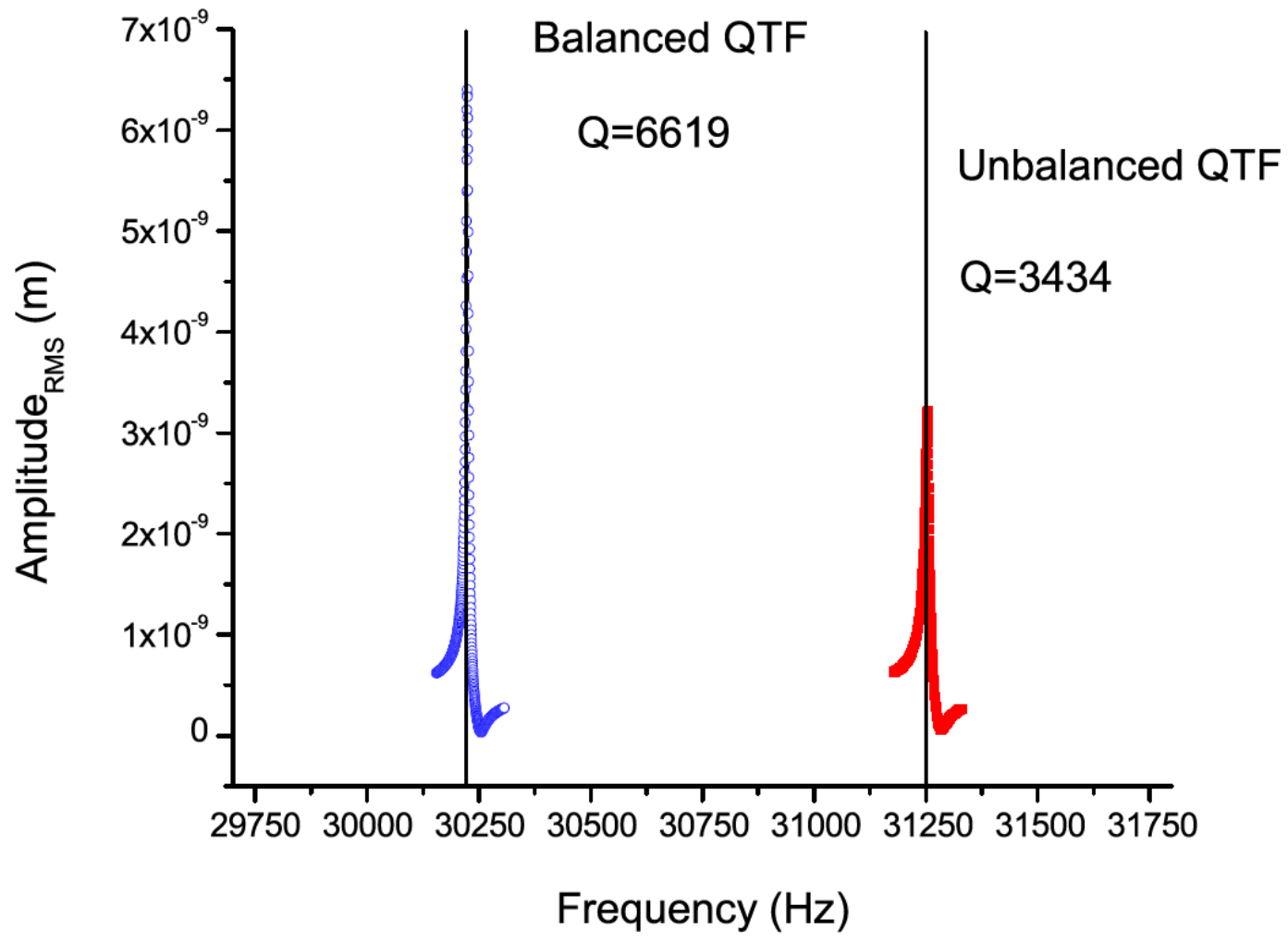

Figure 28. QTF spectrum before and after adding an equivalent mass on the extra tine. 
The results show a larger value of $\boldsymbol{\alpha}$ for the gold tip. A first interpretation would lead to the conclusion that it is necessary to provide more energy to the fiber tip in order to obtain an oscillation of $5 \mathrm{~nm}$ amplitude than for a gold tip. Experimentally it is found the opposite. The Q of the gold tip is 2400 whereas the Q for the fiber tip is 4500 . The discrepancy could be explained by realizing that the equations of the mechanical model used to calculate $\boldsymbol{\alpha}$ do not take into consideration the extra mass of the mounted tip or its elastic constant K.

As stated previously, the mass loaded on the QTF will modify the Q factor [34]. An additional mass placed on the other tine of the QTF will restore the initial Q (without a mounted tip) as shown in figure 28.

Different QTF with different mounted tips have different values for $\boldsymbol{\alpha}$. A high driving voltage was necessary, in some cases, to overcome the limiting $\mathrm{S} / \mathrm{N}$ ratio that tends to be higher when measuring a signal with the USTS.

\subsection{XYZ Piezo Stage}

Although usually not included in the feedback system because of the additional delay it introduces, the closed loop of the $\mathrm{Z}$ piezo positioner can be included in specific circumstances when the imaging speed is not of the utmost importance. In such cases the time response of the $\mathrm{Z}$ piezo positioner constitutes a critical parameter when designing the PID closed loop, because as stated in section 7 it has to have a faster response (shorter time response meaning larger bandwidth) than the PID closed loop. The system would 
become unstable otherwise. The time responses for the $\mathrm{X}$ axis of the XYZ PZJ and the Mad City Labs (MCL) OP-65 piezo-stage (used as a Z scanner in closed loop) are shown in figure 29. The response of each one of the three axis of the PZJ are similar and have a cut off frequency of about $14 \mathrm{~Hz}$ for a step of $8 \mu \mathrm{m}$. On the other hand, the MCL has a cut off frequency close to $170 \mathrm{~Hz}$ for a $6 \mu \mathrm{m}$ step in closed loop.
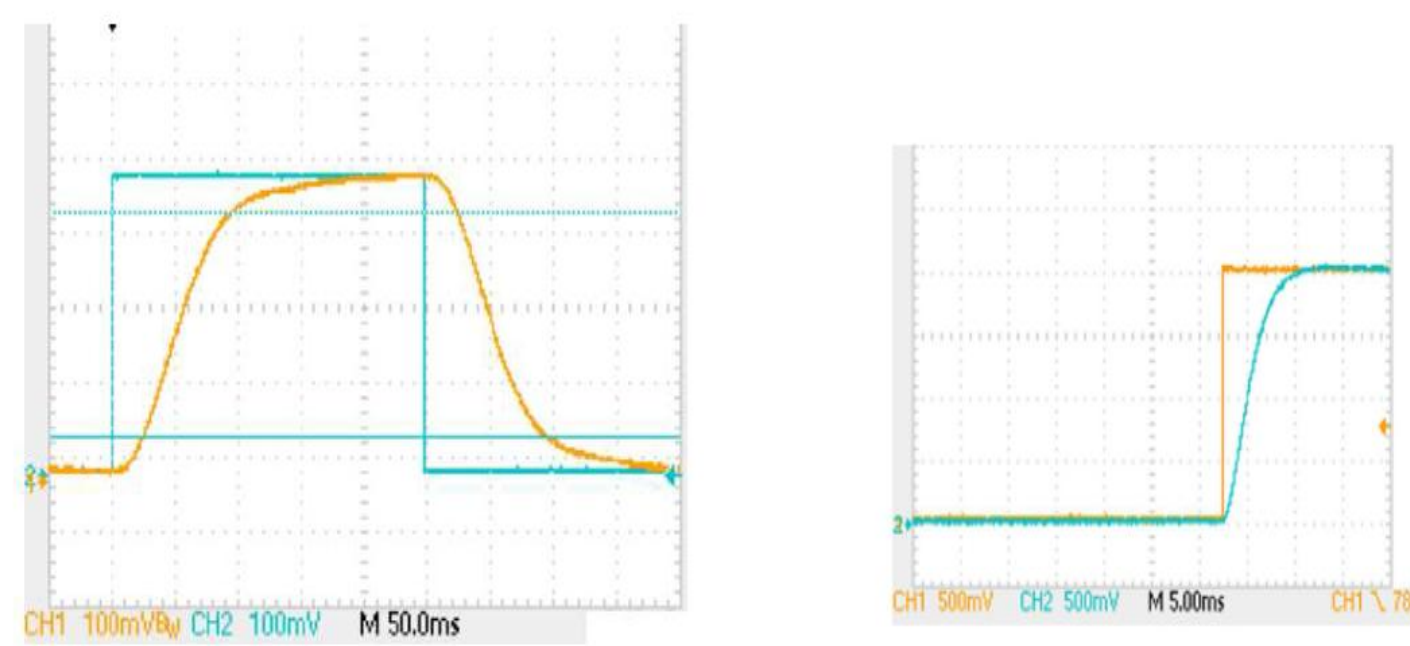

Figure 29. Piezo stages time response.

The PZJ TRITOR $102 \mathrm{XYZ}$ piezo positioner can be used for the XY scanning process as well as a $\mathrm{Z}$ positioner. For the latter, however, it presents a poor scanning speed due to its small bandwidth. Nevertheless, for the purposes of Ultrasonic Feedback it represented the only option since the resonant cavity cannot act as a piezo positioner, because of the interference of the piezo motion with the acoustic signal from the QTF. 


\subsection{PID Control Software}

Feedback control in SPM systems requires fast responses in the order or tens or hundreds of microseconds. A simple calculation, based on a crude model for the topography, will yield an order of magnitude for the required time response of the PID. Since the PID loop needs to respond to interactions in the order of sub-milliseconds, PID loops running in a Windows environment with a latency of a few milliseconds, are inadequate. Virtual instrumentation [68] has appeared as a novel and cost efficient solution for implementing measurement and automation systems. Software development, low costs of PC hardware, and high throughput data rates made possible its positioning as a flexible option that allows scientists and engineers to develop user-defined instruments with specific characteristics. Such characteristics make FPGA cards suitable for SPM controlling. An FPGA is a reprogrammable integrated circuit that can have processing cycles (depending on code size) of a few microseconds. An alternative approach, still in the realm of digital implementation, is found in embedded DSP systems which already have a wide base of users, support documentation and open source projects [66] available. It lacks, however, the robustness of the FPGA since it depends on the clock rates and the effectiveness of the operations the embedded DSP microprocessor can accomplish in a cycle, relying on software to overcome bug problems always present in this type of implementations and when an upgrade is required. Contrary to a DSP, the FPGA is a large interconnected group of gates that perform logic operations, and the programming is less timeconsuming. In this thesis the FPGA PCI-7831R from National Instruments was used. This family of FPGA cards were developed to be used in tandem with the Labview 
FPGA programming environment. Labview, since its conception [67], was designed with a suitable environment for parallel programming. As a result, FPGA technology blends extremely well with it, proving highly suitable for very precise and flexible digital control design and implementation.

FPGA programming in Labview has two distinct environments, the PC mode and the Target mode. A code is written to be executed in the PC and is usually in charge of low speed calculations. A second code is written to be loaded on the FPGA, capitalizing on the resources available in the card and able to communicate with the PC-based program. For this particular program, several techniques were employed in order to achieve the required degree of control for the PID and SPM programs. DMA data transfer, for instance, was included to improve the data transfer rate between the FPGA and the PC and lighten the work of the PC processor. Unlike the interrupts based technique, which stops the FPGA loop until it receives acknowledgement from the PC, DMA allows for the FPGA to keep working without having to stop its process to complete this task. Along with synchronization FPGA techniques, the amount of information able to be transferred without interfering with the PID control is increased. Thus, a single FPGA card enabled the system to acquire at least 5 different channels of information. The number of channels that can be implemented depend on the resources available in the FPGA. Two programs were developed to test the capabilities of our SPM system. The first program is in charge of the scanning, tip-sample distance through feedback with high resolution, drift correction, and data processing for the imaging purposes. The second performs approach and retraction curves before the scanning process to determine the optimum offset. Both are analyzed in the following sections. 


\subsubsection{PID}

As a consequence of mainly being a graphical environment, Labview code would require several pages for a thorough description. The program is here presented in stack form, targeted to a Labview user with a basic understanding the basic programming style. The main program requires three parallel and well differentiated processes: a PID loop, a XY scanning loop, and two separate input and output loops, as shown in figure 30. They are
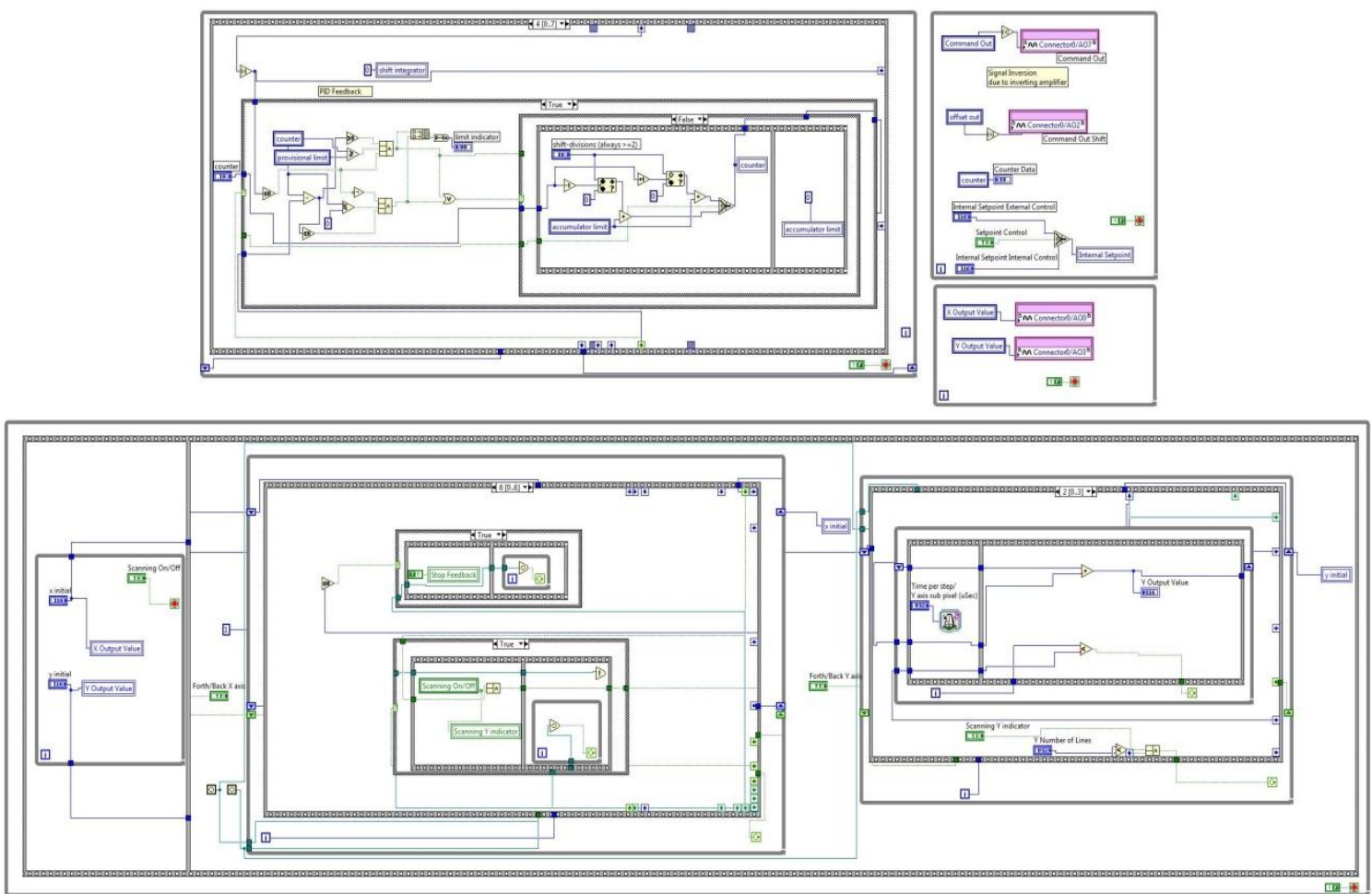

Figure 30. PID and Scanning Labview code diagram.

in constant communication which each other and, when necessary, synchronized to the microsecond ( $\boldsymbol{\mu}$ second). Figure 30 shows the code of the program downloaded into the FPGA. Every block represents a portion of code working independently form the others (parallel programming). They communicate through local variables and synchronization 
techniques (not shown). When programming FPGA code a fundamental difference exists between the code executed in the FPGA card and the code executed in the PC.

Local variables are used everywhere to share data between parallel processes (when synchronization is not essential), and it should be kept as a general rule to avoid true-false programming Cases to save FPGA resources. Only the PID portion is presented.

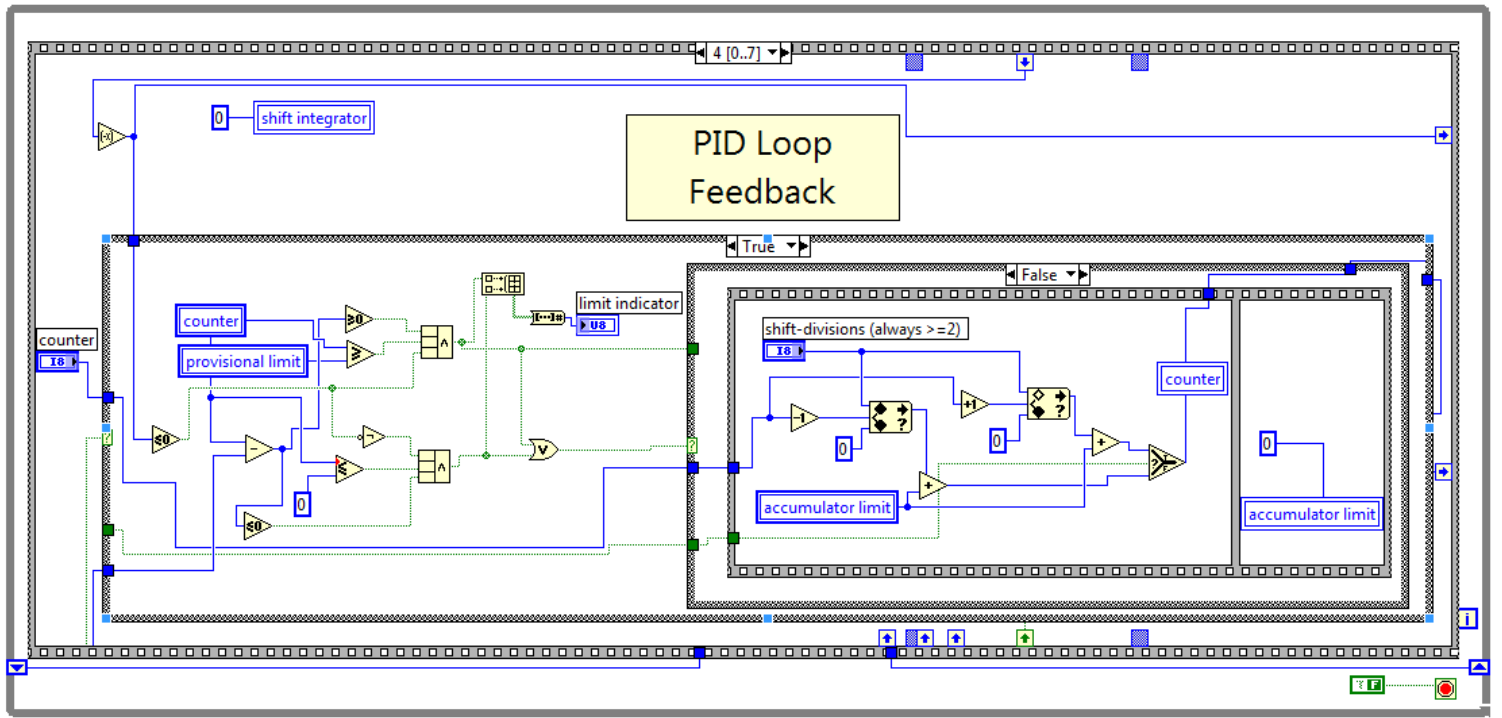

Figure 31. Labview PID code (target).

The card has an acquisition rate of $200 \mathrm{kSamples}$ per second. The period of the PID loop was $5 \boldsymbol{\mu s}$ which corresponds exactly to the acquisition rate provided by National Instruments. This result shows that the main component of the delay is the analog todigital conversion of the card and corroborate that the calculations made by the logic gates of the FPGA play minor part. The PID was implemented from Labview's PID 


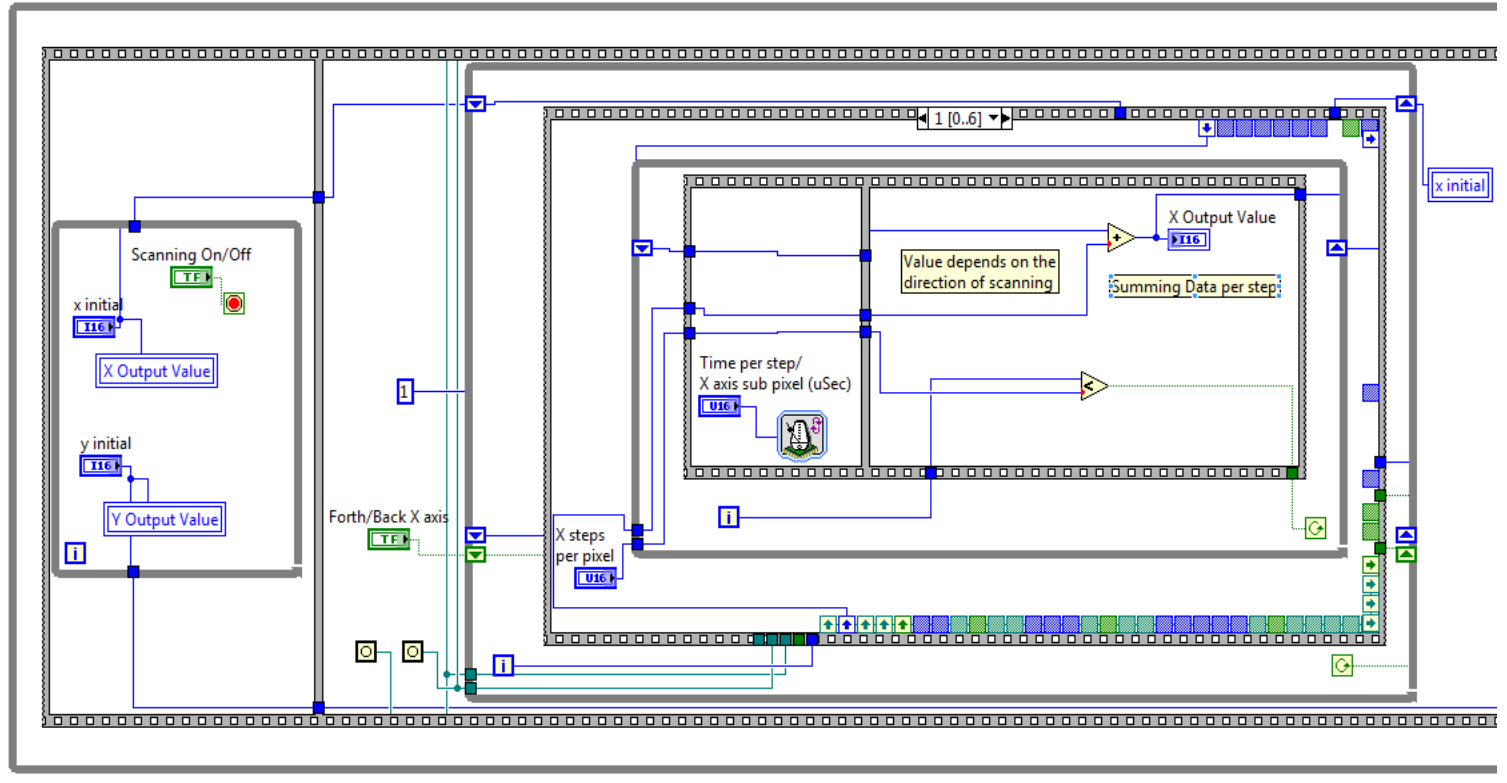

Figure 32. XY Scanning Labview PID code (target).

PID routine, improved to meet the requirements. A modification of the PID module (Labview Package) made possible the increased resolution of the integral and proportional gain. As a consequence, we had smaller responses to changes in the error signal leading to increased PID sensitivity. The resolution of the output driving voltage represented a major problem. If used directly, the output of the FPGA card will give 150/65565 Volts/step (because of the High Voltage amplifier available). This meant a resolution close to $2.4 \mathrm{~nm}$. Adding the DAC's noise, close to $1.2 \mathrm{~nm}$ (FPGA card specifications sheet), a total resolution of around $3.6 \mathrm{~nm}$ is obtained, excluding the noise from the High Voltage amplifier electronics. This low resolution will prevent the system to attain an stable locked state or even to ever reach it. The modification allowed the implementation of the High Resolution Z stage Control and Drift Correction. The firs stage deals with the acquisition of the PID parameters and contains the modified PID 


\section{Labview module.}

The code showed in figure 31 decides whether or not the offset for the High Resolution $\mathrm{Z}$ Motion implementation (explained in detail in the next section) has to move from one region to the other. Figure 32 and figure 33 show the calculation of the $\mathrm{X}$ and $\mathrm{Y}$ scanning values, respectively.

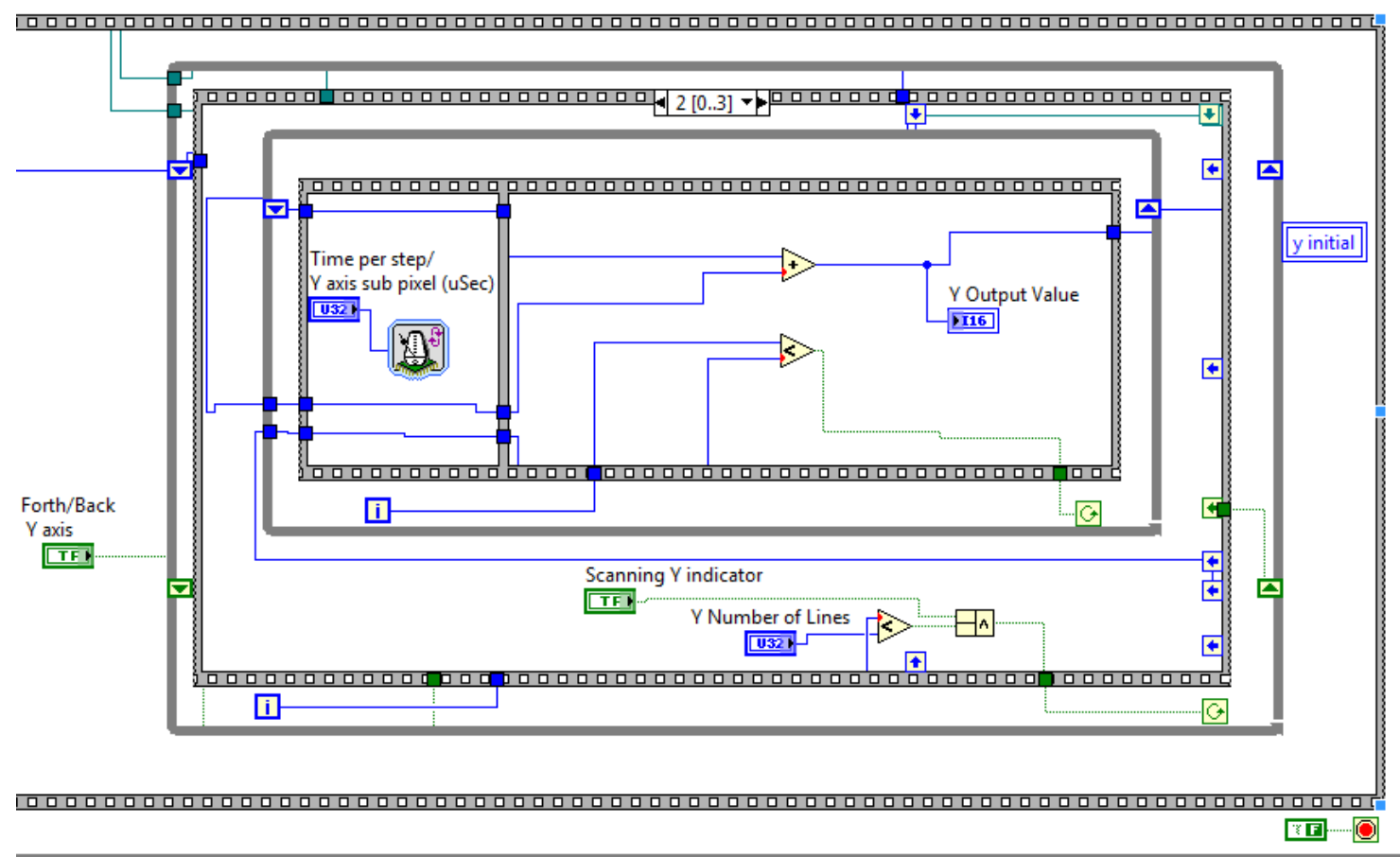

Figure 33. Y scanning (target).

The front panel of the VI running on the PC is showed in figure 34. 


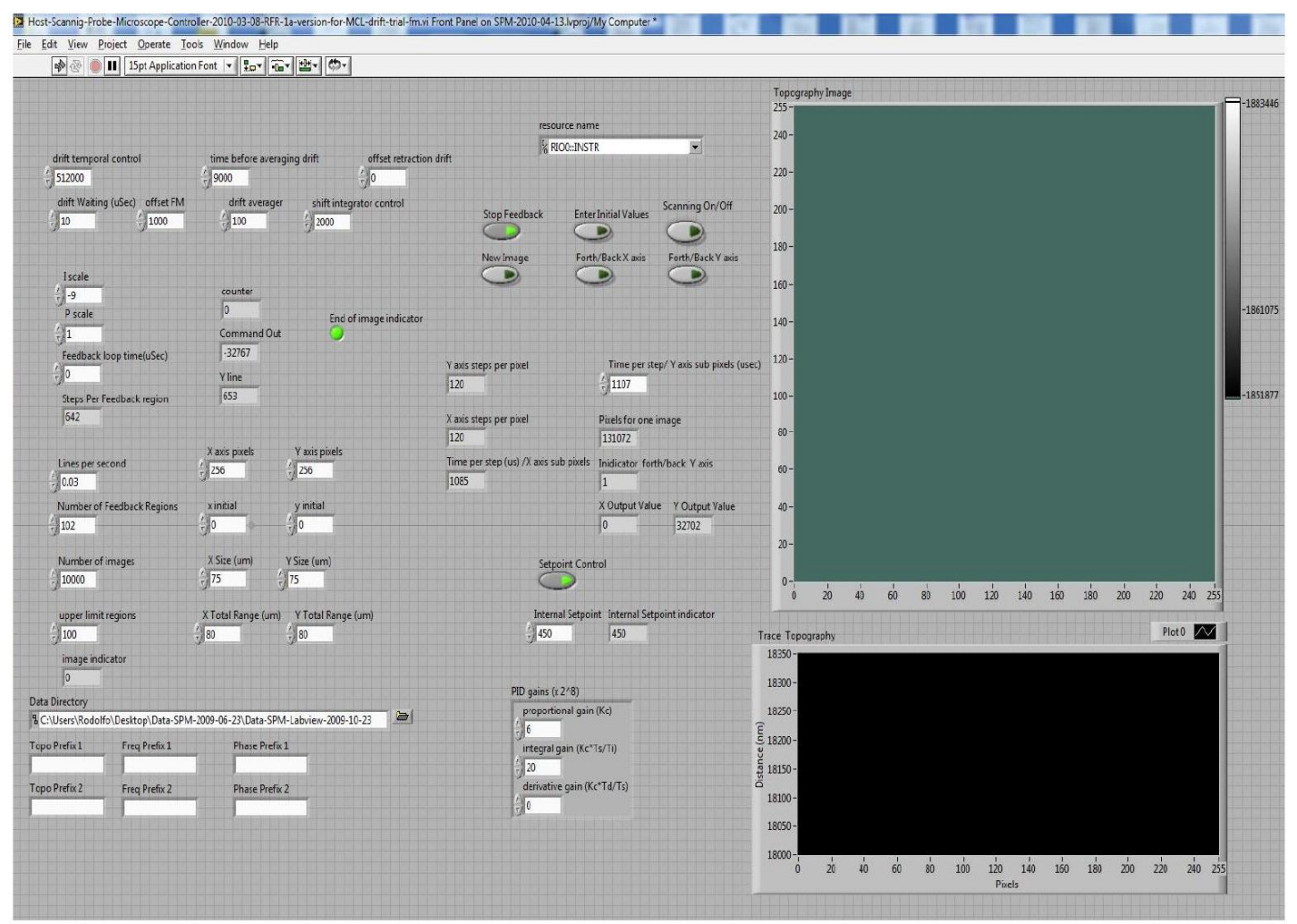

Figure 34. Labview Front Panel imaging Control (Host).

\subsubsection{Approach and Retraction Curves Program}

This program uses the programming technique explained above, but with two major differences. The program, although still continuously tracking the signal provided by the QTF, no longer tries to keep it constant, i.e., has no PID control. 


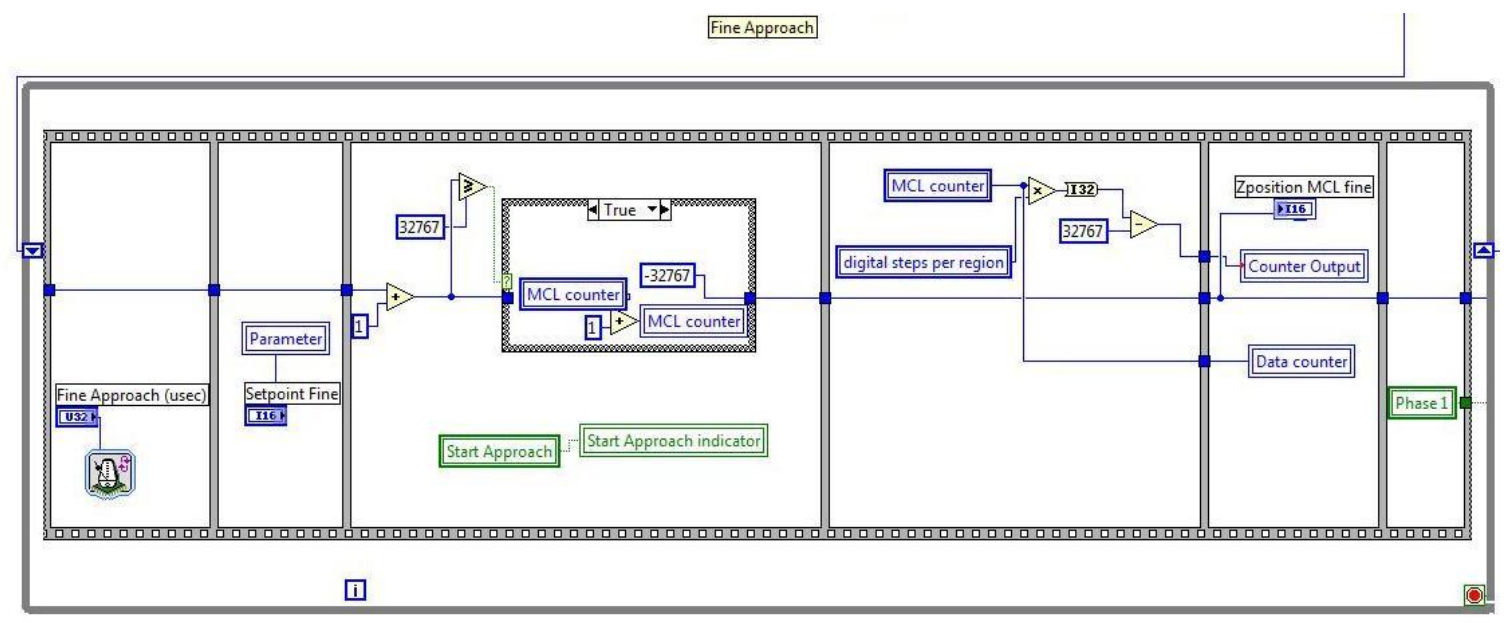

Figure 35. Approach and retraction program basic loop code.

The software merely tracks the signal in order to decide whether the probe should be approaching or retracting and what velocity it should use. In addition, it has a preapproach stage for finding the surface.

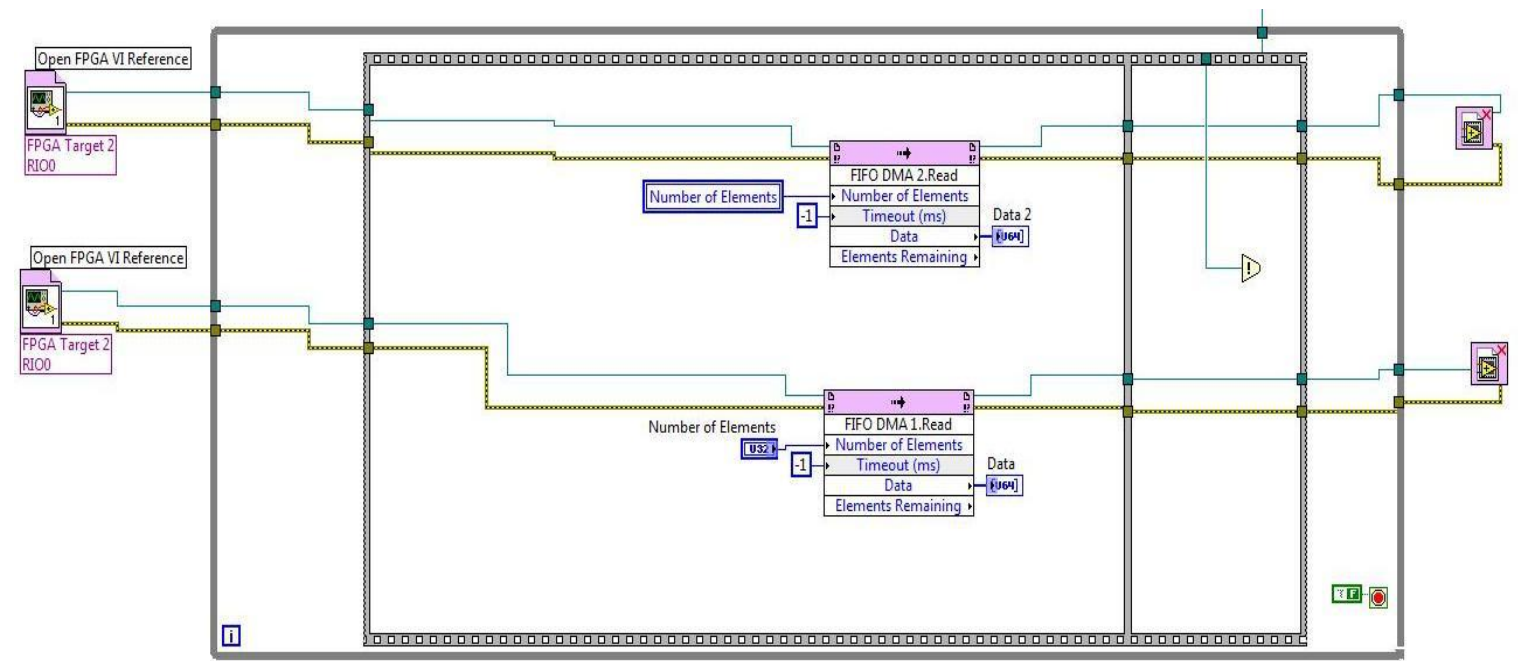

Figure 36. Approach and retraction program host code. The diagram shows the DMA type data transfer. 
Consequently, the smallest change in the signal (much less than the noise level) will make it retract at the maximum velocity. Figure 35 shows the main part of the program. This automation permits performing tens of approach and retraction curves with high reproducibility. This program also permits the manual control of the distance between the probe an the sample.

The next section deals with the proposed solution for the High Resolution problem.

\subsection{High Resolution Z Motion}

The voltage to displacement ratio $\mathbf{v}$ (in Volts/nm) determines the resolution of the piezotube motion. Figure 37 shows how this ratio impacts the position control. Low position resolution could translate into feedback problems, simply because the signal given by the PID output would never correlate with the proper tip sample distance. Resolution of the PID gains are also a factor, and they will be addressed later. The lower $v$ is, the more likely is that electrical noise will diminish the sensitivity of the feedback positioning system. The minimum driving voltage step (the voltage at which the displacement of the piezo is greater than the indeterminacy of its position which is buried in noise), when all other factors are optimal, is mainly determined by the noise level of the driving signal. The higher $\mathbf{v}$ the higher the resolution of the motion. The PZJ TRITOR $102 \mathrm{XYZ}$ system and the MCL have 150/80 Volts/um and 150/65 Volt/um respectively. In order to increase this parameter a High Resolution Driving Stage was implemented. 

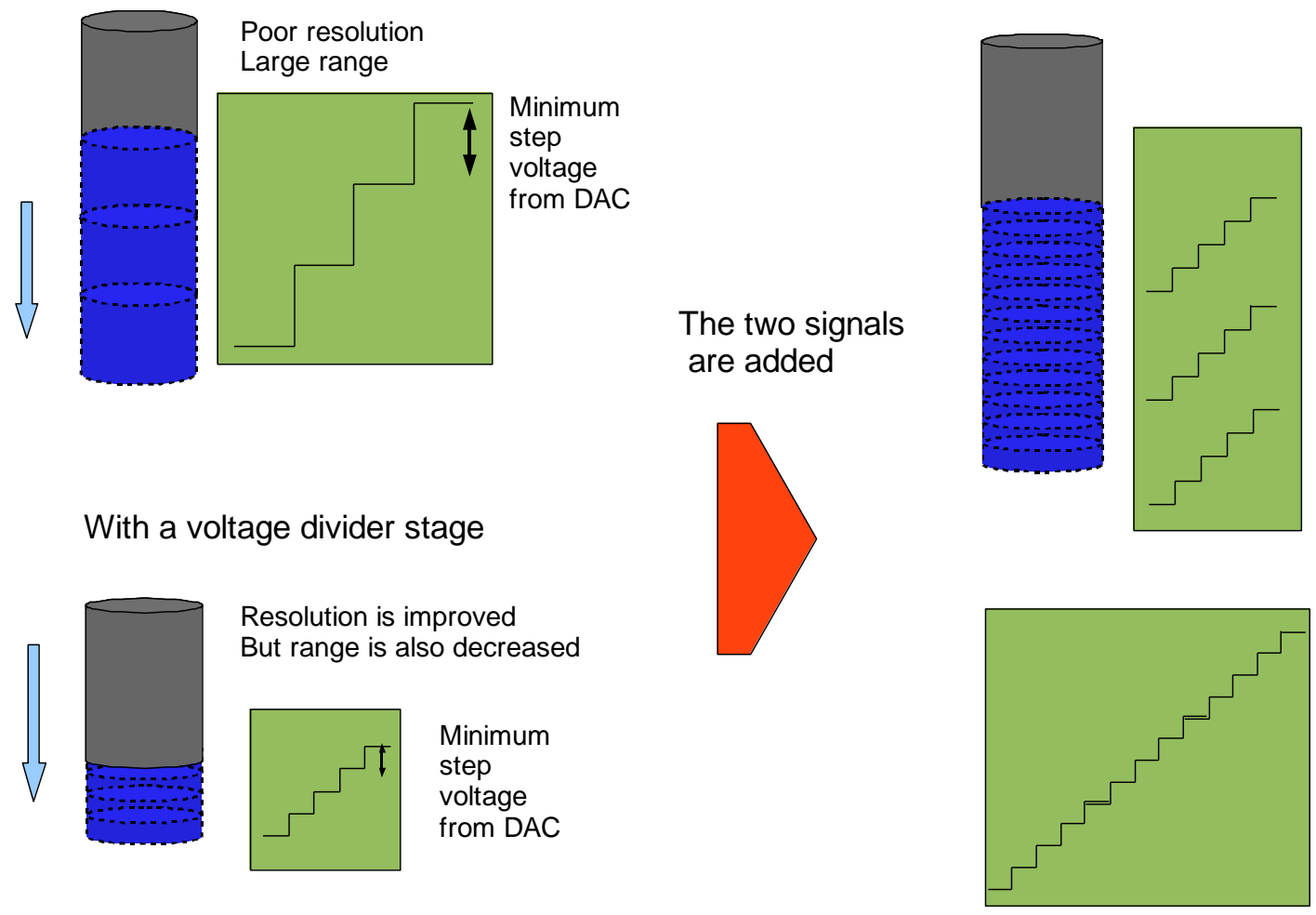

With a voltage divider stage

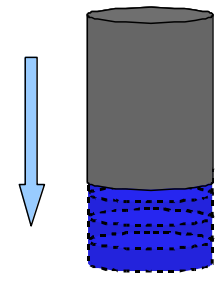

Resolution is improved

But range is also decreased

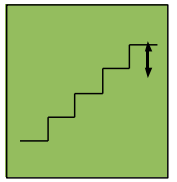

Minimum

step

voltage

from DAC

Figure 37. High Resolution $\mathrm{Z}$ control problem.

The PWM and low pass filter solutions have been widely used for the increase of DACs resolution, from 16 bits to 20 bits and even 24 bits. The solution proposed in this thesis employs two signals that are added by an op-amp (additive op-amp) circuit.

The whole range of the piezo-stack was divided in regions and with the addition of an analog part, a summing amplifier, the desired resolution was attained. The current set up has a subdivision of 100 regions giving a theoretical resolution of $0.036 \mathrm{~nm}$ when using the 16 bit DAC from output channel from the FPGA. Noise from the amplifier and the resistors will worsen the actual resolution. 
As presented in figure 37 a voltage divider stage would indeed increase the position resolution, but the whole range of the driving signal would be proportionally diminished. To overcome this problem a two signal stage is implemented. The original signal, with an increased resolution due to the voltage divider, controls the position, but a second signal is added to offset the final signal by an amount equal to the full range of the first more sensitive signal. Note that synchronization between these twosignals has to be carefully implemented.
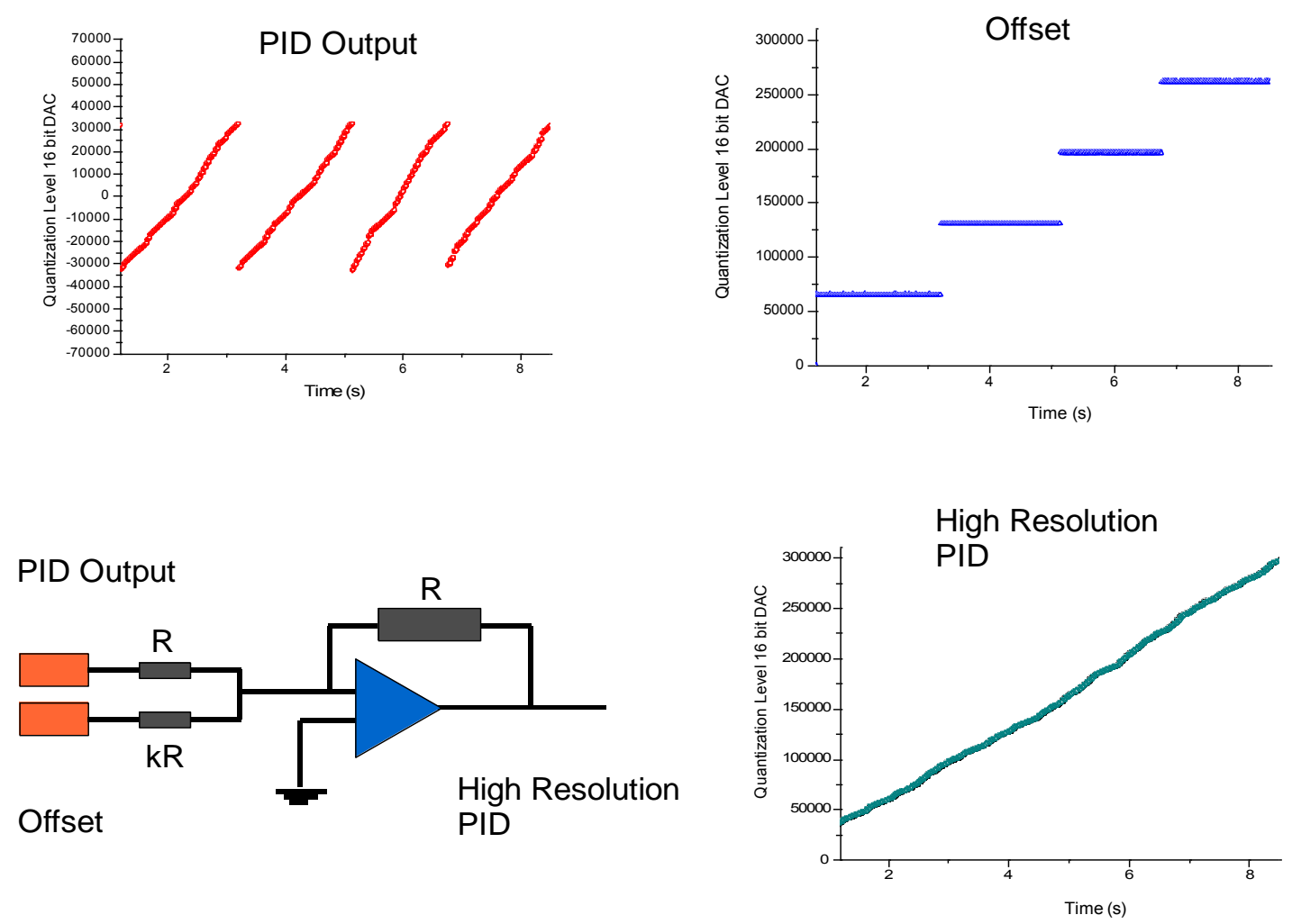

Figure 38. High Resolution $\mathrm{Z}$ control solution.

The FPGA code was modified to return the original signal (PID output) to its minimum value as fast as possible, once the maximum value was reached. In synchrony with this 
reset of the first signal, a second output shifts the signal. The ratio between this two signals is set by the ratio between the summing resistors in the analog summing amplifier circuit. Figure 38 shows how this was implemented.

Figure 38 also shows the experimental data and the High Resolution PID circuit. In the case of the PID output, the values are given in the quantized values for a 16 bit DAC, which gives a range from -32767 to 32767 counts. Here k represents the ratio between the two signals, and also the number of divisions the full range of is made of. For the Offset and the High Resolution PID graphs the values are k times the 'real' DAC output value, in order to show the effective resolution obtained with this technique.

\subsection{Tip Preparation}

Chemical tip fabrication for SPM can be roughly divided in static or dynamic chemical etching [30] and laser heating (pulling and bending) [31]. The static chemical etching method setup implemented in this laboratory was chosen for this work. It requires submerging the optical fiber in a $49 \%$ solution of HF. The tube etching method was introduced by Zenobi in 1999 [65]. The setup implemented (figure 39) can etch tips with an average diameter of $40 \mathrm{~nm}[32]$.

Its insulating properties produce an accumulation of charge on the tip. A coating process 

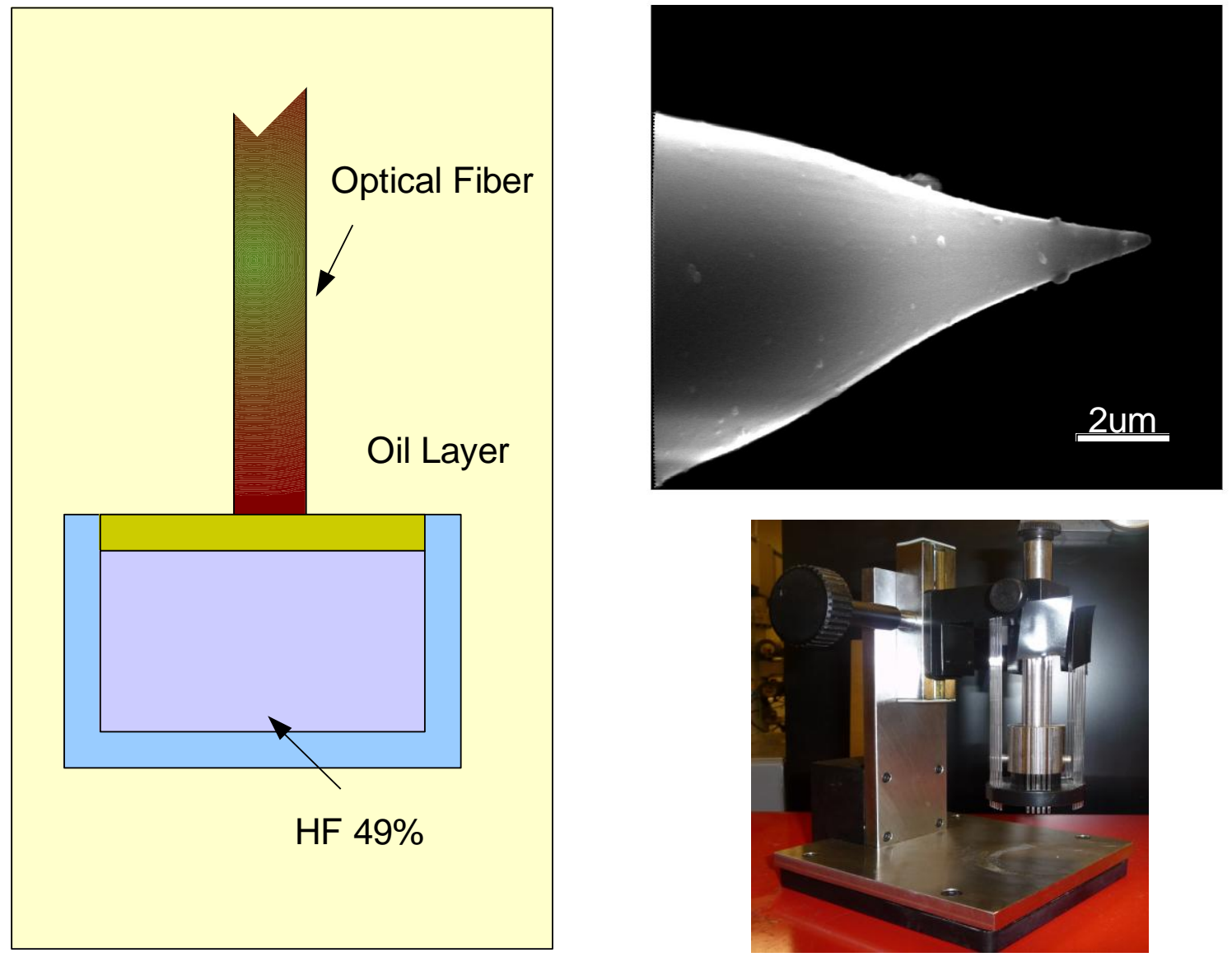

Static Etching Setup

Figure 39. Optical Fiber tip obtained by HF static etching.

will make the tip surface conductive and redistribute any charge present over the whole coated area. The coating increases the tip-radius about 20 to $50 \mathrm{~nm}$. Gold metallic tips fabricated through electrochemical etching, a process that involves anodic dissolution (gold wire) in an aqueous solution of $\mathrm{NaOH}$ [33]. Gold density is about ten times larger than silica's, increasing the asymmetry of the QTF, thus decreasing its resonant frequency as well as its Q. Both gold and coated etched fiber were used in this work. 3.6.

\section{Noise Management}


Mechanical, electromagnetic and acoustic signals are the most important sources of noise for SPM systems. A passive optical table reduced the possible mechanical oscillations coupled to the SPM stage. Acoustic noise does not pose a significant problem. Nevertheless, it is recommended the use of acoustic isolators and absorbers. The laboratory is located in underground facilities. A single BNC cable with one end free and the other attached to an oscilloscope performing a FFT showed peaks at low frequencies, as shown in figure 41 .

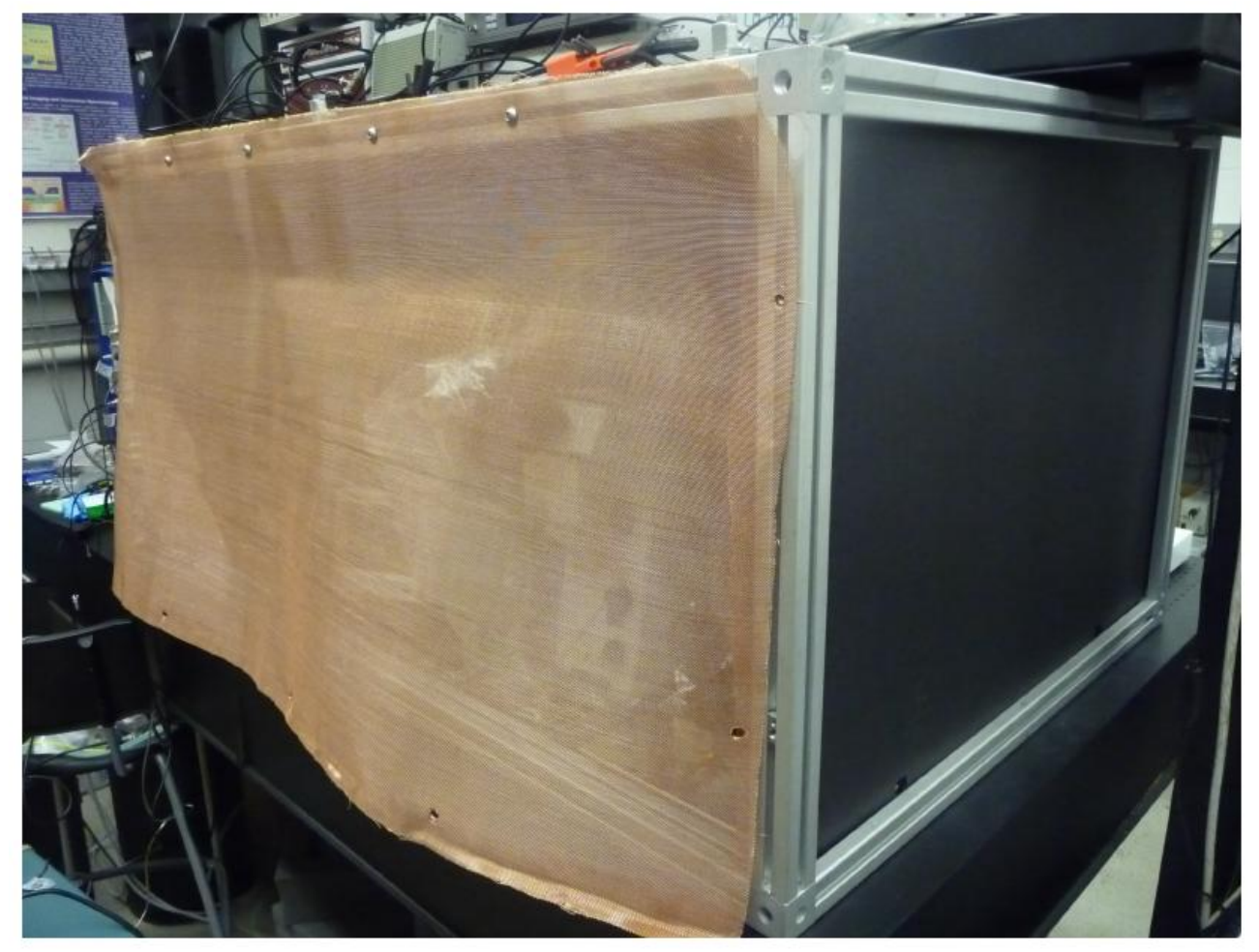

Figure 40. A copper metallic mesh completely surrounds the Al-extrusions frame.

Electromagnetic noise from laboratory fluorescent lights have a signal away from 32 $\mathrm{kHz}$, thus with little if any impact on the signal of interest. Low frequency signal couples 
with the QTF signal at all times and it seems to come from equipment used in the building, permeating the whole laboratory. In order to isolate our system from this electromagnetic noise a Faraday Cage was built.

The grounding was implemented by connecting the cage to the outlet ground and the optical table, which has a considerable stainless steel mass. Figure 41 shows the spectrum before (left) and after (right) placing the same BNC cable inside the cage.
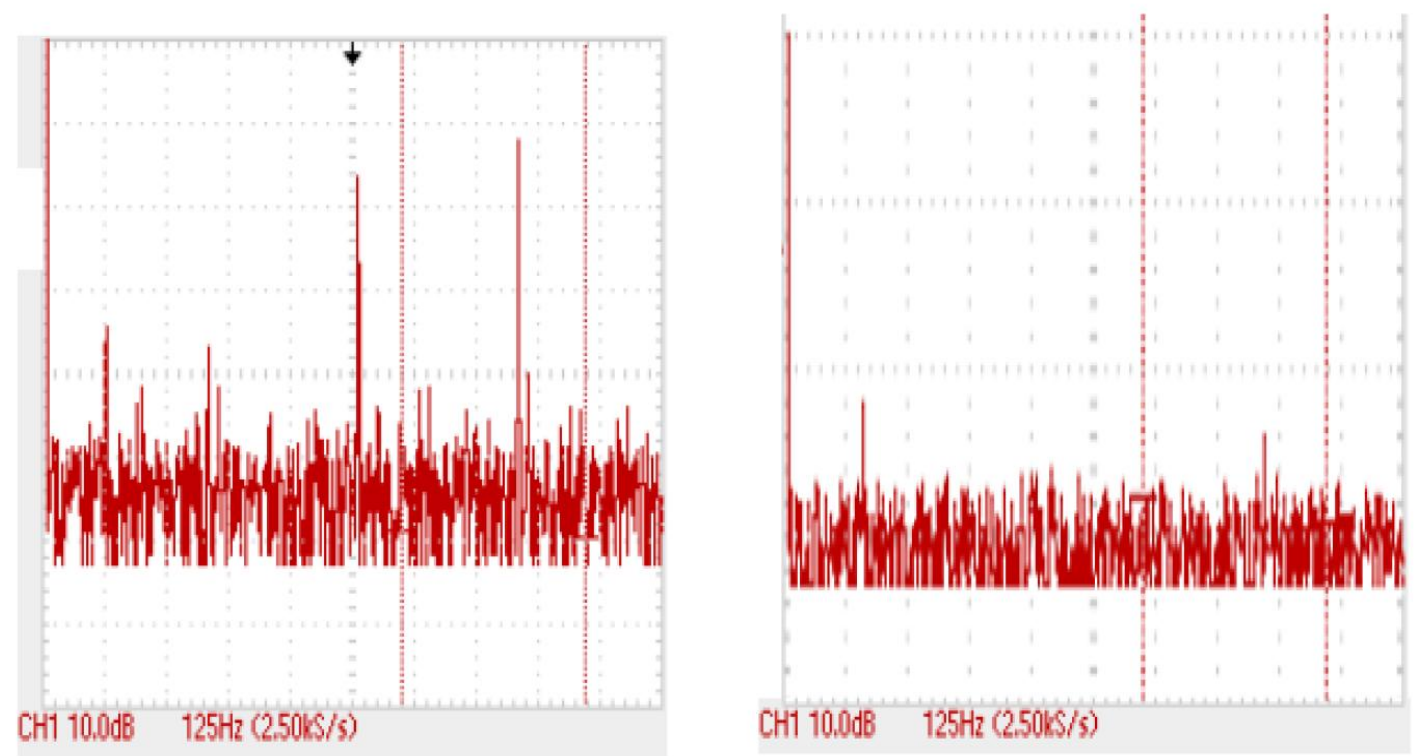

Figure 41. Noise Spectrum before (left) and after (right) the Faraday cage implementation.

\subsection{Ultrasonic Detection}

The USTS, as with any piezoelectric based device, produces a current proportional to the 
displacement of its sensing area. This active sensing area is carefully coupled to the region of interest, namely, the surface of the acoustic resonant cavity. The specific position was experimentally chosen as the region with highest signal. The USTS has a sensitivity of approximately $1 \mathrm{~nm} /$ Volt [63] at $32767 \mathrm{~Hz}$. The mechanical coupling between them represented a challenge. Unless they have been subjected to special processes, surfaces always have roughness at the micrometer scale, making effective contact area between two surfaces objects very small. For this purpose vacuum grease was used as a coupling substance to fill the microscopic gaps between the contact surfaces.
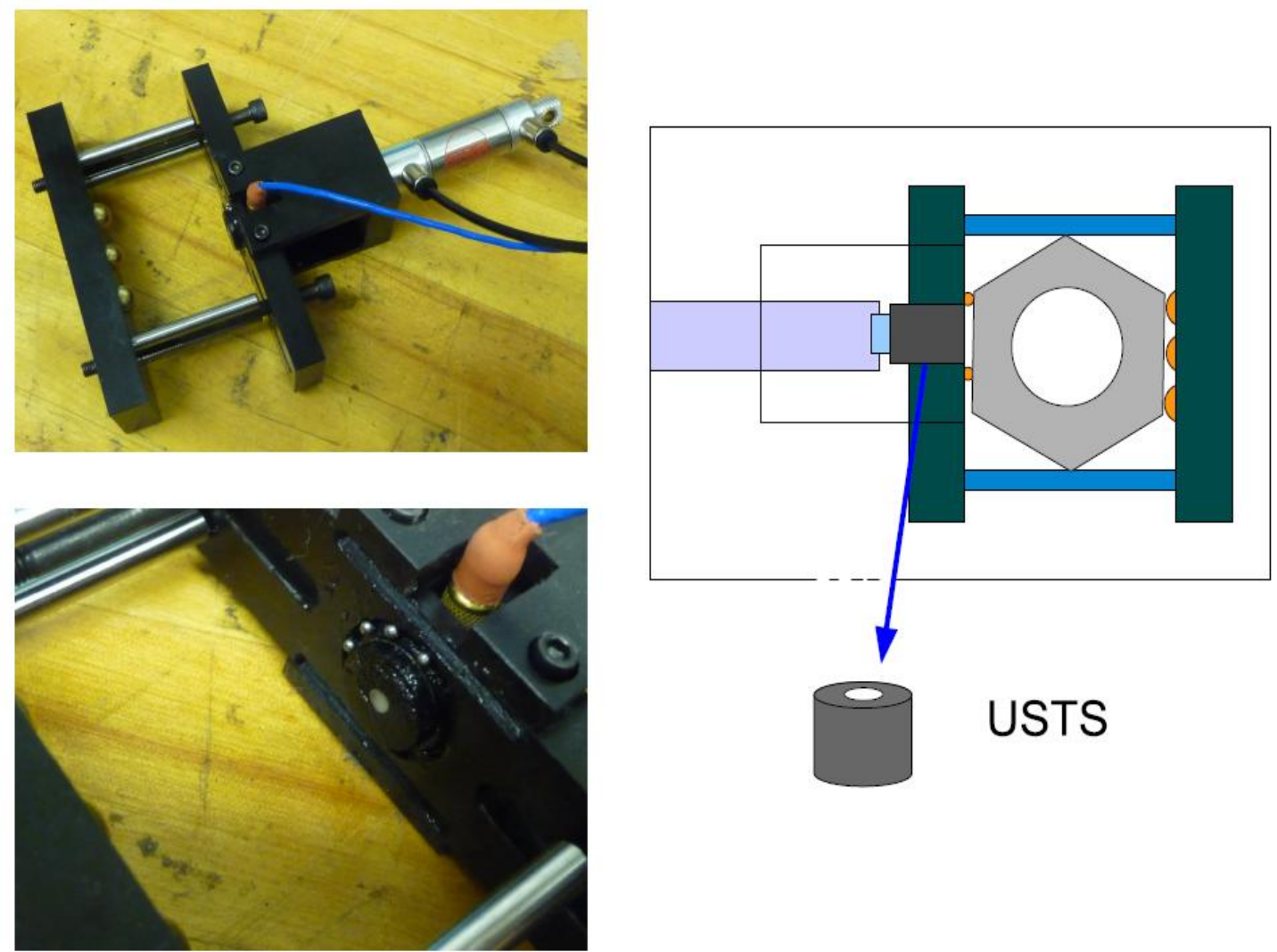

Figure 42. USTS holder implemented to improve control of the contact pressure. 
At first, a spring clamp was used to mount the USTS onto the WG but it offered poor time stability in addition to limited pressure control on the sensor. It was noticed that the acoustic signal was very sensitive to the applied external force making absolutely necessary to find solutions for better control of the pressure and thus ensuring mechanical stability and repeatability of the measurements. A USTS holder was designed and built. This holder was designed specifically to minimize any contact area with the WG itself, with exception of the active area, and avoid any distortion of the resonant mechanical system. Ball bearings were used for this purpose as shown in figure 42. Temporal stability improved and repeatability was consistent. By sweeping the excitation signal's frequency around the RF of the QTF the following graph was obtained,

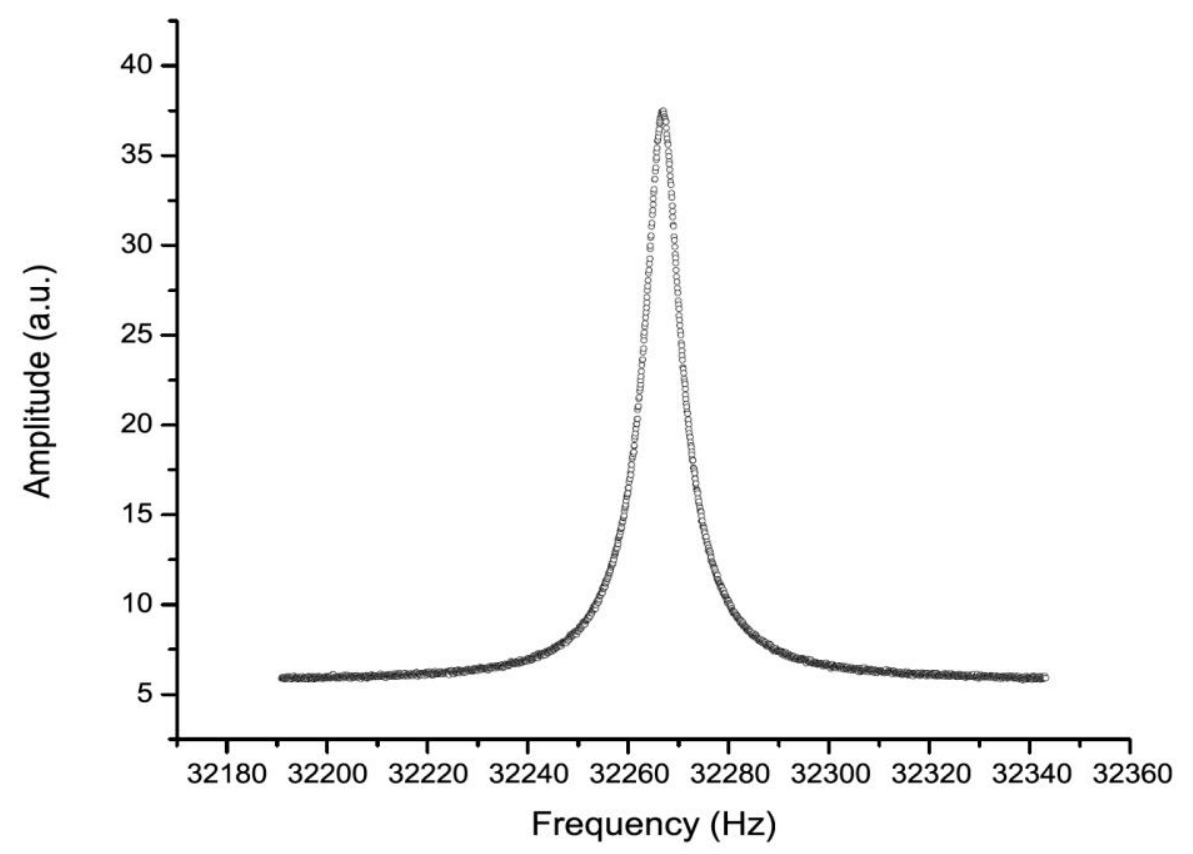

Figure 43. Frequency sweep USTS measurement

The spectrum is symmetric around the QTF's resonance frequency, which differentiate it 
from the direct electrical QTF response.

This setup was used to validate the partial results from the simulation. An spectra of both QTF and USTS is presented in figure 44. After each frequency step the system waited $100 \mathrm{~ms}$ to measure the signal in order to avoid ringing.

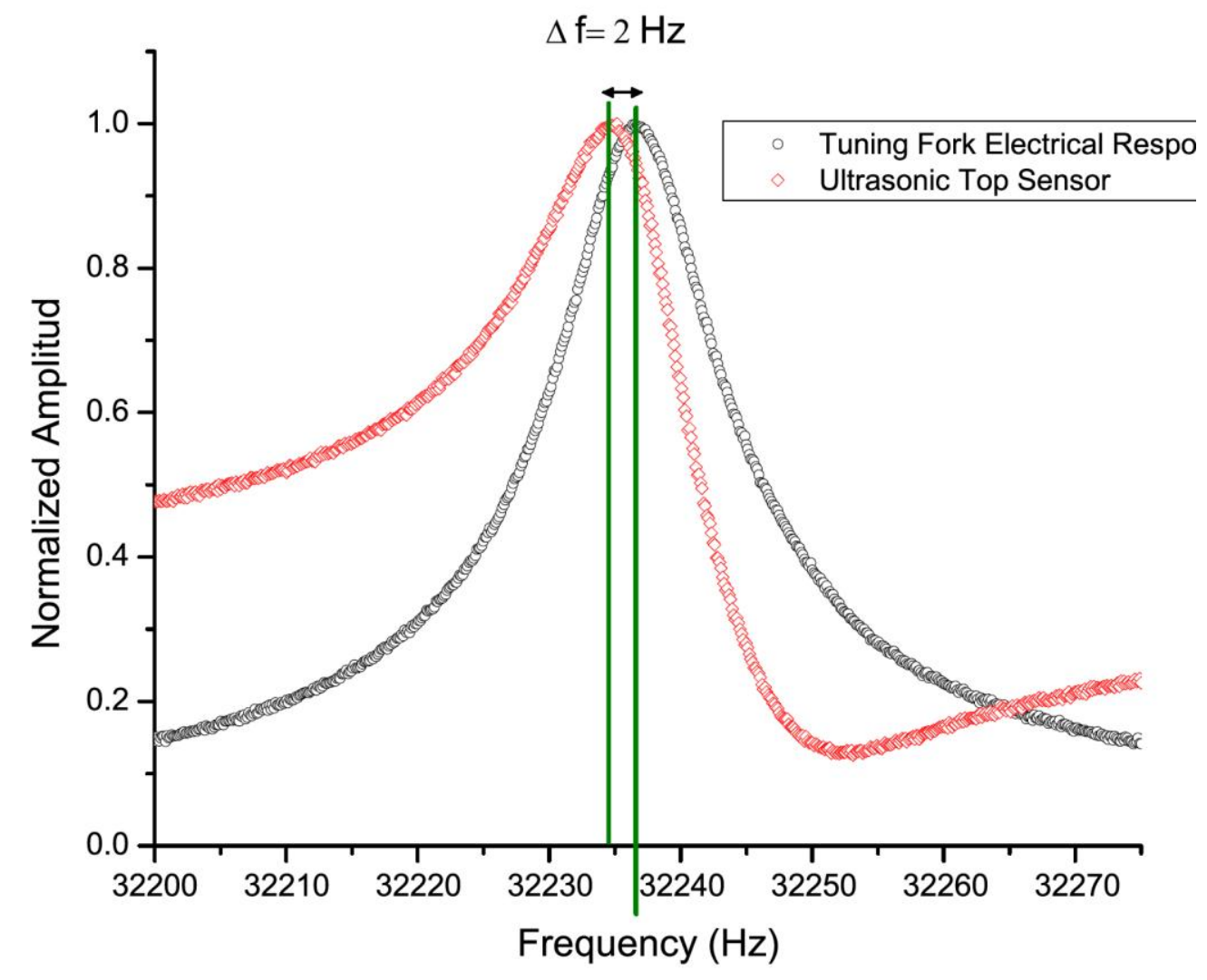

Figure 44. Comparison between the spectrum of the USTS and the QTF signals. There is a shift of 2 $\mathrm{Hz}$ in their resonant frequencies.

The acoustic response from the front face of the WG was mapped using the USTS. Every region was carefully measured several times to discard mechanical coupling effects. The results are shown in figure 45. 


\section{Frontal Hexagonal Face}

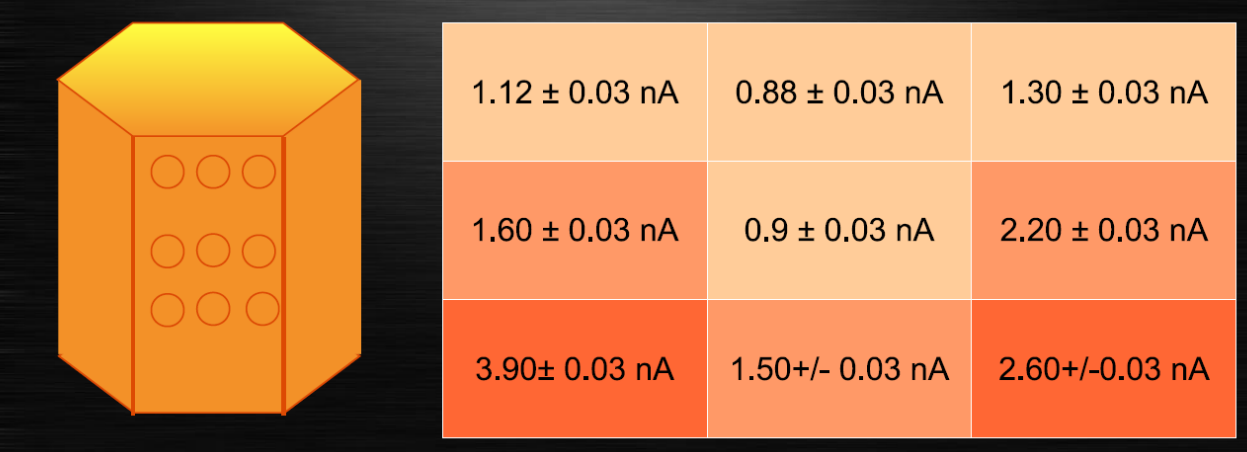

Figure 45. USTS measurements of the Front Face of the WG. 


\section{Chapter 4}

\section{Acoustic Resonant Cavity Simulation}

Many mathematical models are described in terms of PDE and integral equations. Analytical solutions of these models are often very involved and many times not practical at all. FEM is a computational technique that finds approximate but very accurate solutions to the PDEs. Presently, FEM refers to a number of mathematical methods with common characteristics. In the FEM an equivalent formulation for the PDE needs to be found (variational formulation). The infinite dimensional problem is then projected into a finite dimensional subspace (Galerkin method) which represents the best approximation to the true problem. By using a piecewise linear subset, the resulting algorithm is accurate and efficient. Common computational techniques (Runge-Kutta, Euler's Methods, Newton, etc) are used to find the solutions for every region in the mesh. Compared to other techniques, FEM shows great flexibility for different boundary conditions and geometries. FEM was used to calculate the resonant modes of the WG. In this chapter the details of the simulation will be explained and preliminary results presented. 


\subsection{Frequency Response Whispering Gallery}

As a first step the SPM head was modeled in Solidworks as shown in figure 47. The model was then exported as a CAD file to the $\mathrm{CM}$ package which was used to find the Stationary Frequency Response of the modeled Acoustic cavity. The diameter of the inscribed cylinder is $5.81 \mathrm{~cm}$ and the interior cylinder is $3.81 \mathrm{~cm}$ in diameter. The height is $6.88 \mathrm{~cm}$. The physical parameters involved in the simulation are the Young's modulus, Poisson's ratio, the thermal expansion coefficient and the density. These parameters were entered for the parts of the SPM head, namely stainless steel, polymer (Macor) and Lead Zirconate Titanate.

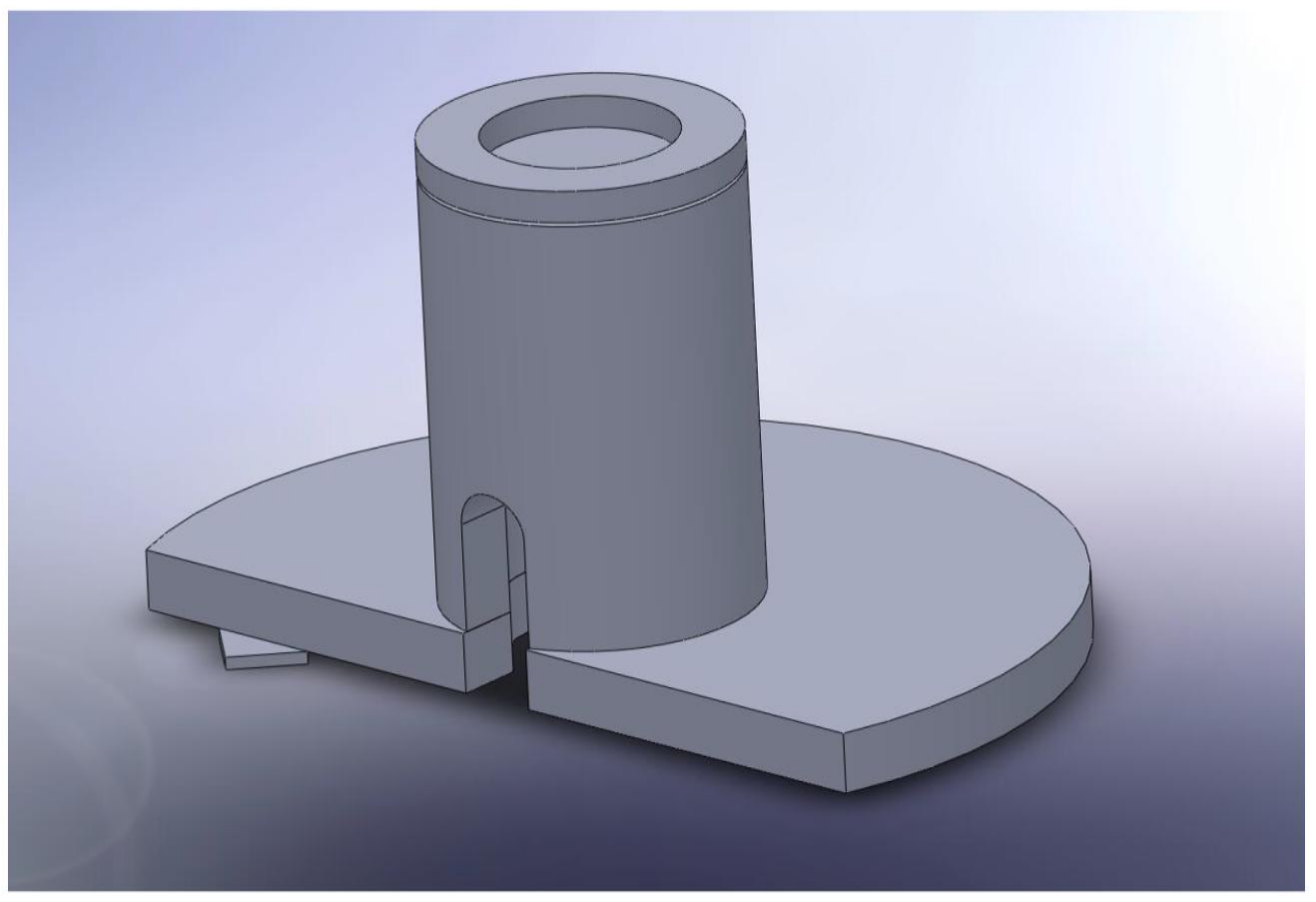

Figure 46. SPM head designed in Solidworks.

The size of the mesh (or the number of regions that are used to solve the Stationary 
Frequency Response problem) determines the processing time. For the mesh shown in figure 47 it takes approximately 150 minutes of computer time. This time will increase with an increased number of regions (finer mesh).

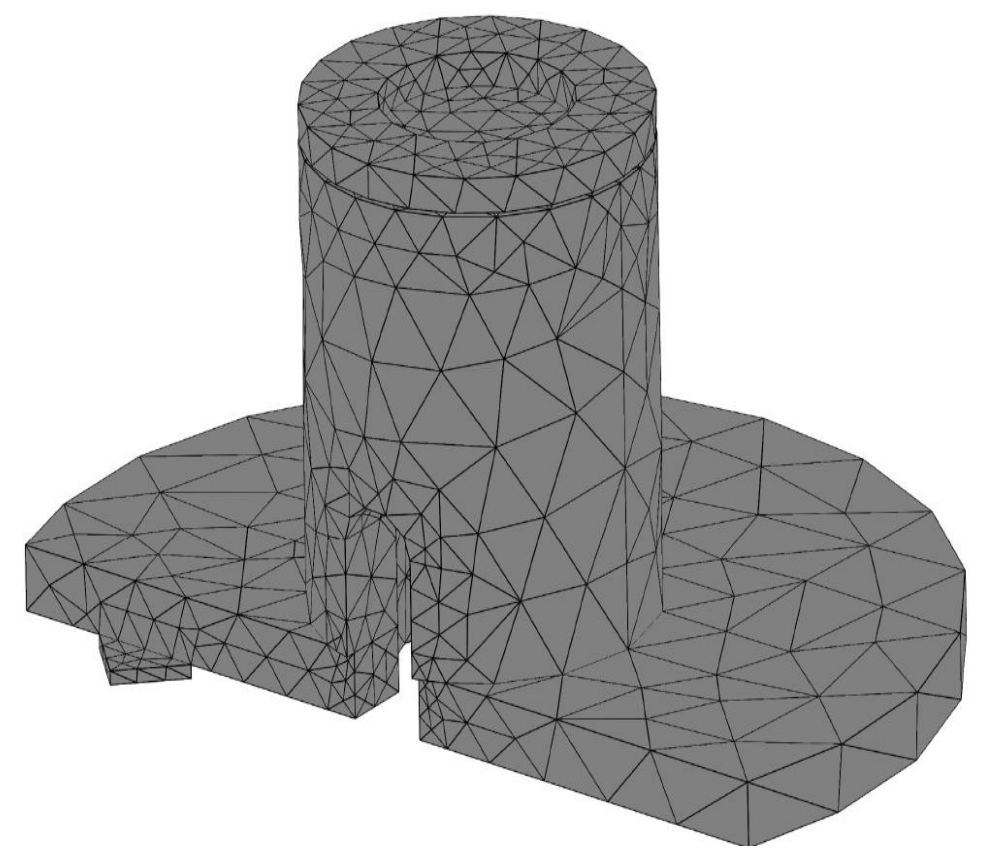

Figure 47. SPM head with the mesh pattern for solving the PDE equation with the COMSOL package.

The CM package solved PDE equations for the modeled SPM. At first, large sweeps were performed, in a range between $5000 \mathrm{~Hz}$ and $45000 \mathrm{~Hz}$, with $50 \mathrm{~Hz}$ steps. Subsequently, we used a top-down process to find the intervals where meaningful peaks were found. Finally a range between 25000 and 35000 was selected. As a first calculation the cavity was excited with a piezoelectric material attached to its base, exciting its resonant modes. An example of one steady state solution is shown in figure 48. 


\section{Cylinder SPM Head}

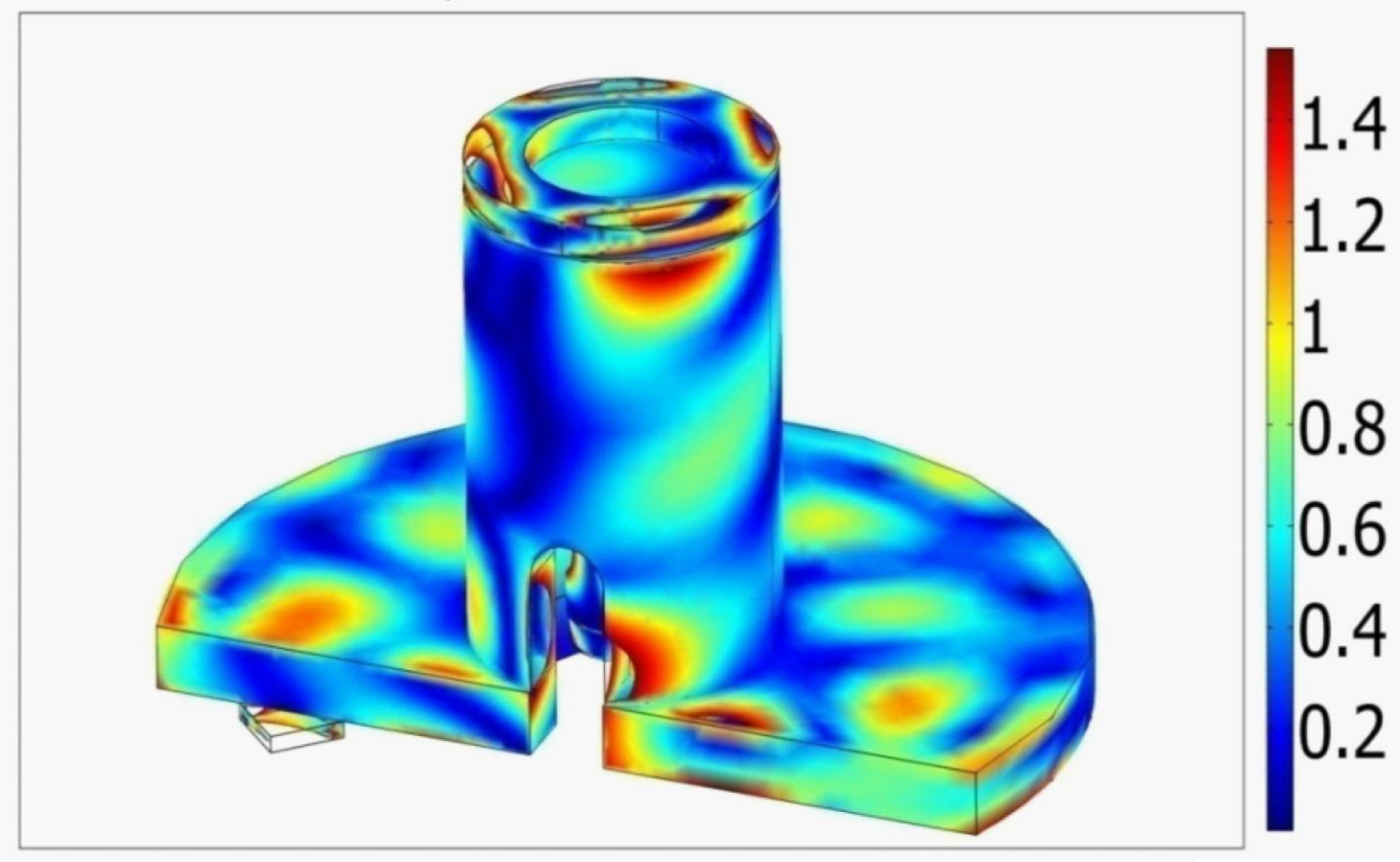

Figure 48. Solution for the SPM head steady state frequency response at $32546 \mathrm{~Hz}$. The values for intensity are in arbitrary units.

Figure 49 shows the frequency spectra found for the topmost right corner area of the frontal half-face of the Whispering Gallery, The preliminary results for the cylindrical are presented in Figure 50. Since the component of the oscillation perpendicular to the SPM head surface is the main contributor to the signal measured with the acoustic sensor, it was the chosen response parameter.

The different curves represent different regions on the surface of the SPM's head. From the graph it can be concluded that the peaks are independent from the region where the perpendicular component of the oscillation is measured. 


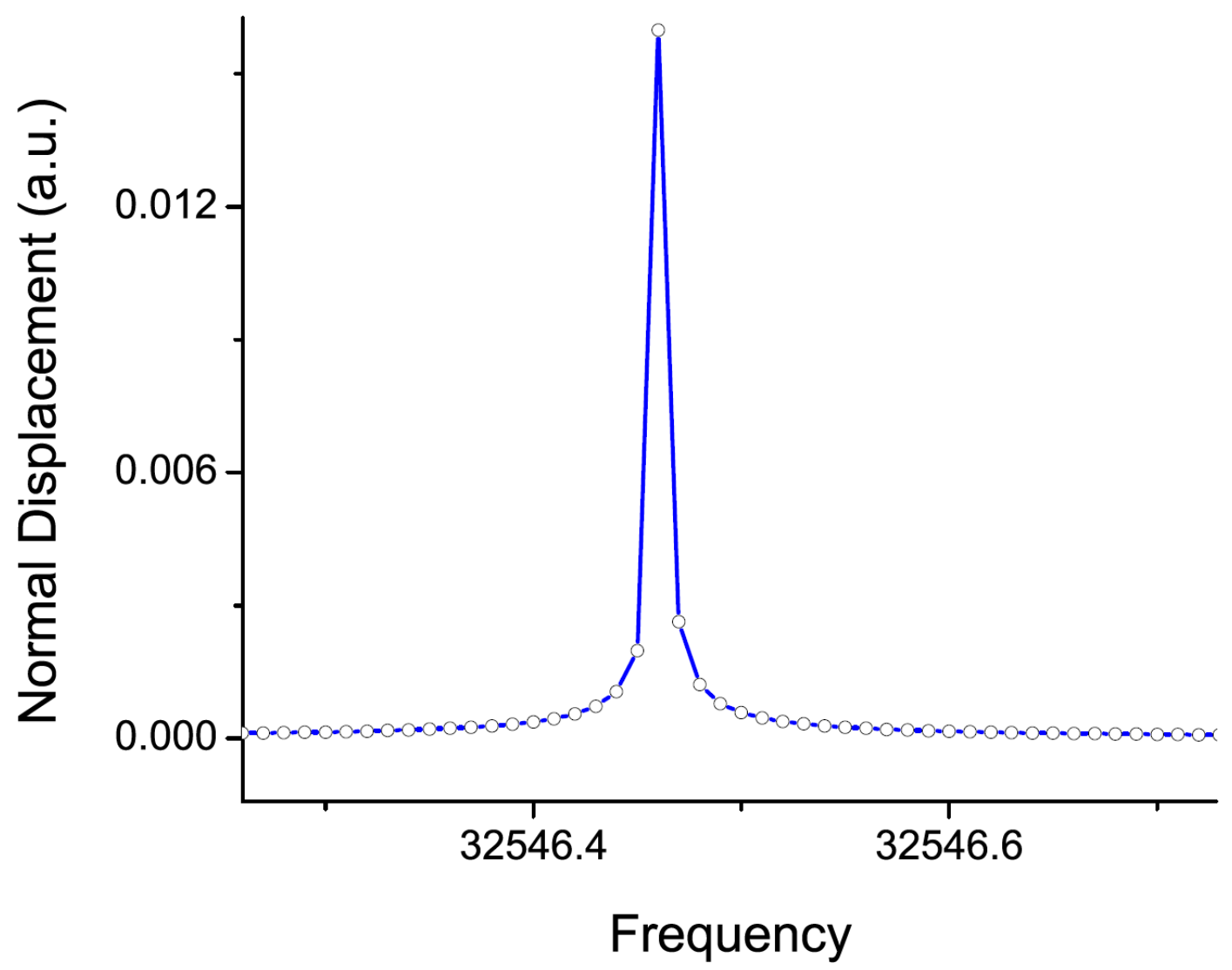

Figure 49. Frequency spectrum of a region from the frontal face of the Whispering Gallery.

These preliminary results show the trend observed in the resonant modes as the dimensions of the WG increase. Figure 50 also presents the results for a 5\% and $10 \%$ increase. Encircled are main peaks elated to specific dimensions of the cavity. As expected, with increasing dimensions the frequency of the resonant modes are decreasing. According to these results, is possible to modify the modes of the resonant cavity by modifying the WG dimensions, and find the appropriate geometry to produce a high gain in the frequency region where the QTF has its resonant. This will improve the USTS's SNR and will allow implementing the frequency and phase modulation feedback 
control in the future.
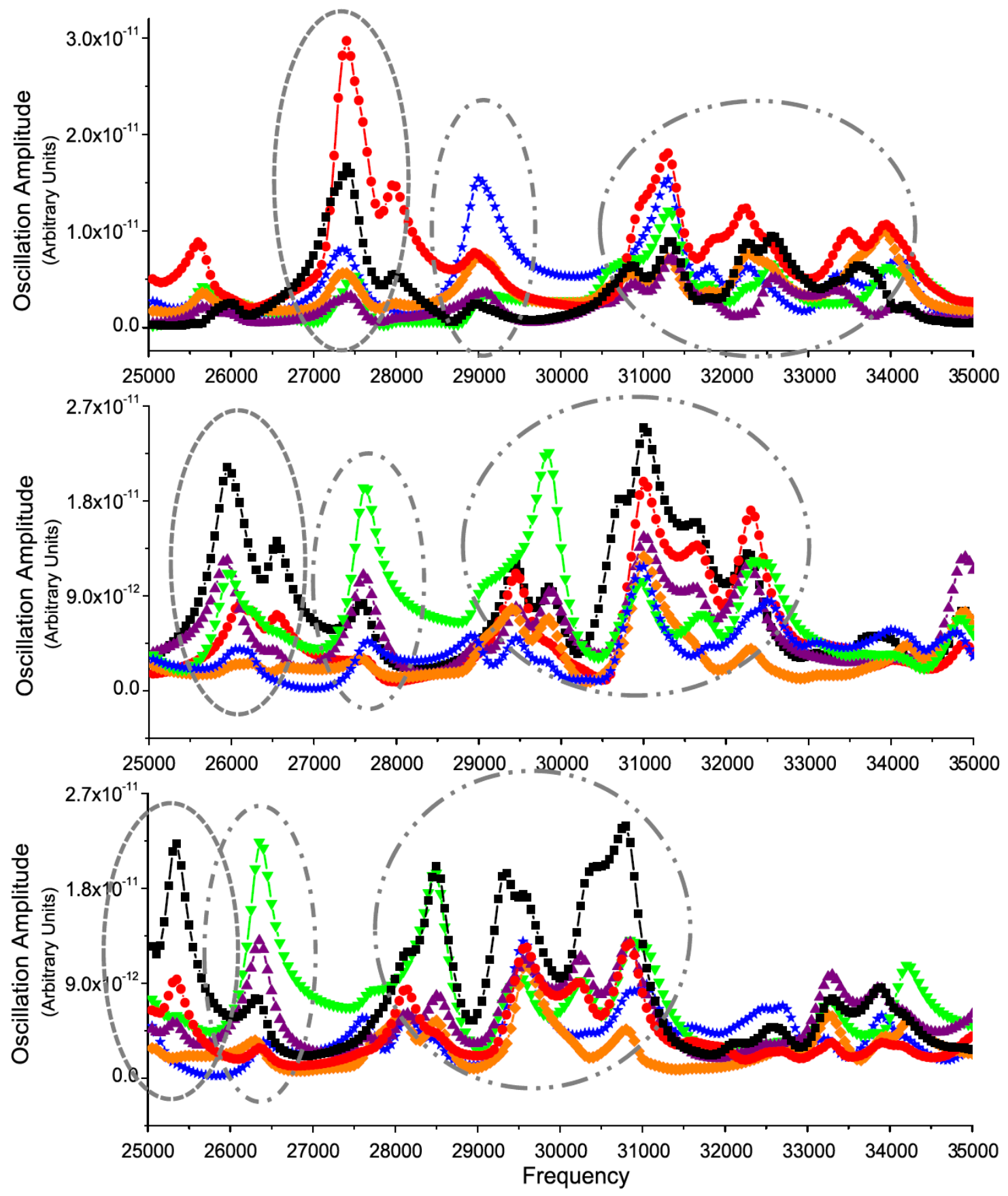

Figure 50. Comsol solution for the SPM head. Solutions for different positions on the cylinder's surface at $0 \%$ (top), $5 \%$ (middle) and $10 \%$ (bottom) increments. 


\section{Chapter 5}

\section{Results}

Approach and retraction curves obtained with the Shear SPM are presented. Before every imaging process an approach and retraction curve was taken. The objective was to test the resonant frequency shift for a specific level of dampening, obtaining the relation between the AM mode response and the FM mode response when they engage in feedback. It also provided information on how the FM technique could be use in the analysis of force interactions in the contamination layer.

Images taken with AM mode are presented. FM modulation was not used in tandem with the WGAS feedback technique due to SNR considerations. The system needs to be improved to allow for this type of detection. 


\subsection{Approach and Retraction Curves}

An analysis of the interactions between the tip and the adsorbed contamination layer was the objective of a series of approach and retraction curves. The homemade software developed in Labview code and downloaded into the FPGA, as described in chapter 3, was in charge of the displacement velocity control. Parameters such as the angle between the sample plane and the tip, tip and sample geometry, sample cleanliness, etc. are critical factors in this process and were carefully controlled. The sample was submerged in IPA and cleaned with an ultrasonic cleaner. The tip-sample angle was maintained close to 90 degrees. At first, and at an approximate distance of $15 \mu \mathrm{m}$ from the surface, the preapproach setting is enabled and the software takes control. The program monitored the oscillation amplitude of the QTF, while approaching with constant velocity set between $10-20 \mathrm{~nm} / \mathrm{s}$. After the surface has been found, the probe approaches at a rate of $1 \mathrm{~nm} / \mathrm{s}$ (as specified from the output control signal) stopping the approach immediately after the amplitude reaches $45 \%$ of its initial non interacting value (away from the sample) and immediately retracting with the same speed. A graph for the observed general behavior is presented. The sample used is a silicon wafer that had $50 \%$ of its surface coated with the poly(N-vinylcarbazole) (PVK) polymer. The tip is a gold tip etched by the electrochemical etching procedure described in chapter 3. Figure 51 shows the approach on silicon. 


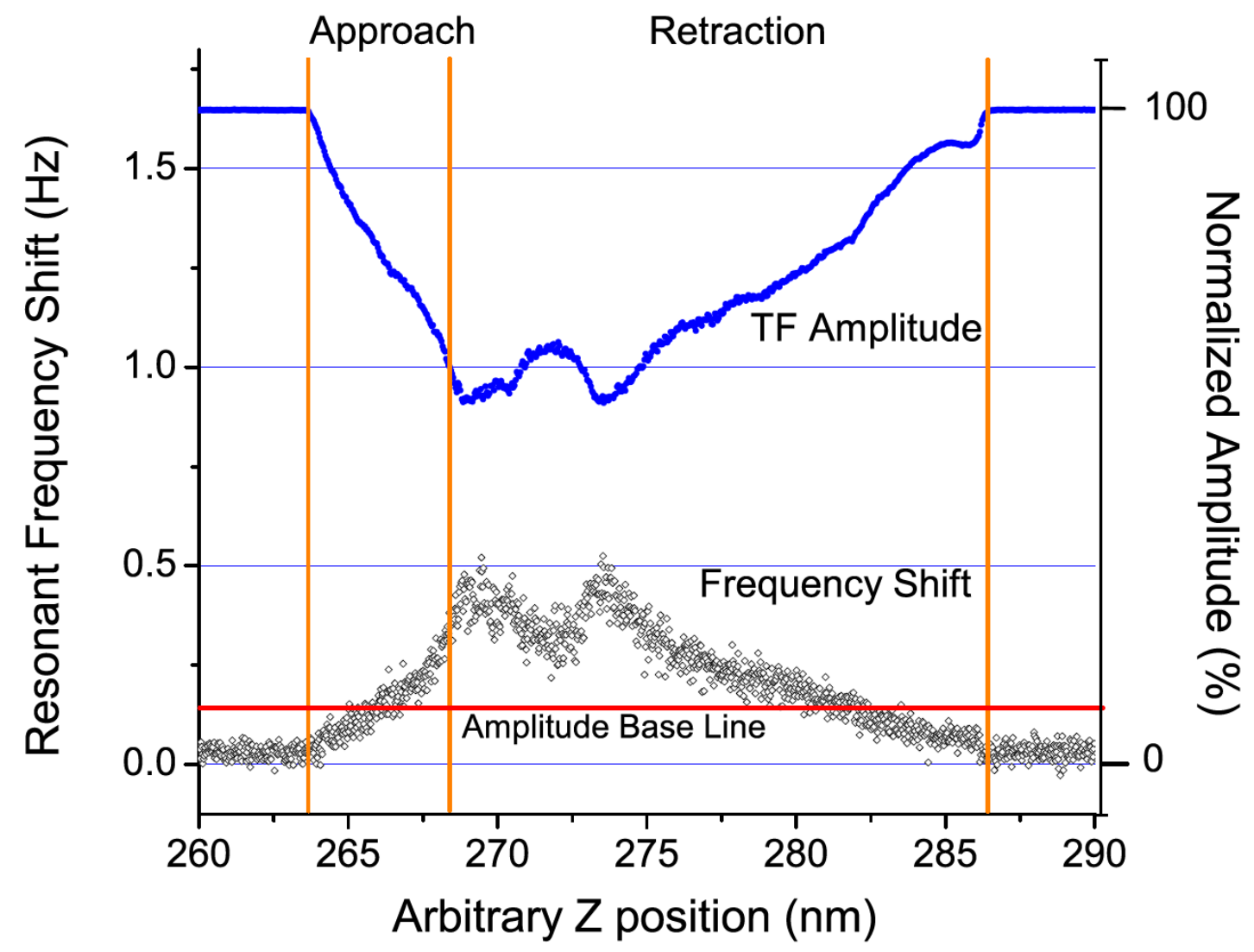

Figure 51. Approach and retraction curve for a $45 \%$ offset in silicon with a gold tip. The depth of the layer for a $45 \%$ amplitude dampening is $\mathbf{5 n m}$ whereas the retraction requires $18 \mathrm{~nm}$ to attain the maximum signal.

The graphs are divided in two regions, the 'approach' region and the 'retraction' region. The curves presented are plotted in such a way that the position in the region labeled 'approach' is decreasing from left to right and in the regions labeled 'retraction' the position is increasing from left to right. The case of silicon surface shows a $5 \mathrm{~nm}$ depth and $19 \mathrm{~nm}$ for the retraction. The interactions are both conservative and dissipative at the beginning. The larger displacement found in the retraction is apparently due to the meniscus formation after the tip has been inside the water layer. A better look at the 
central region (while retracting) shows the inverse correlation between the resonant frequency shift and the amplitude. Oscillations in the order of $5 \mathrm{~Hz}$ are noticeable, but it is not clear whether they are caused by tip-layer interactions (or tip-surface interaction) or just coupled noise from other sources.

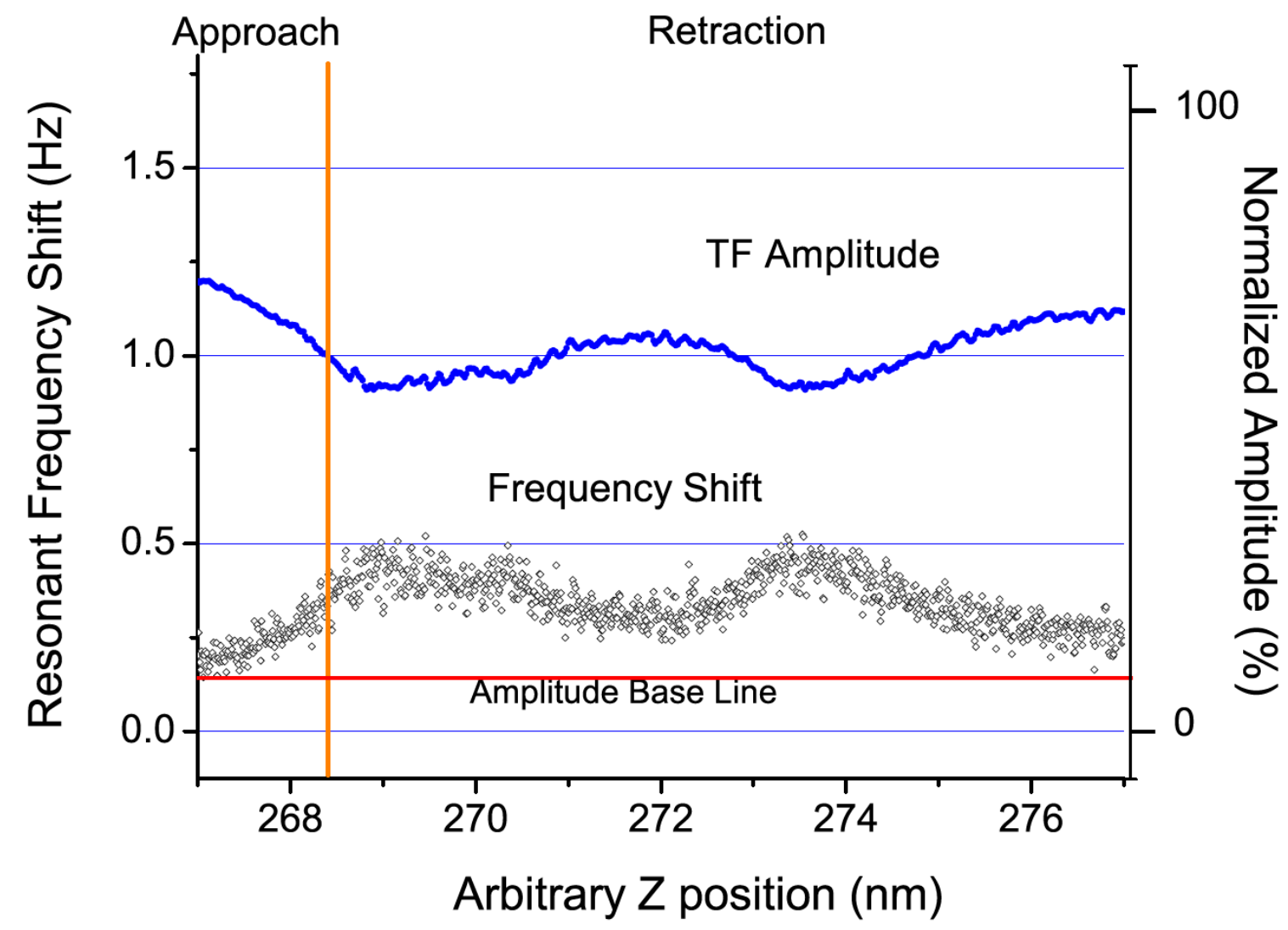

Figure 52. A closer look at the approach process of figure 51.

Finally the retraction region is shown in figure 53. In the far right the exact position at which the tip is released from meniscus is observed. There is a sharp change in the amplitude and resonant frequency level. 


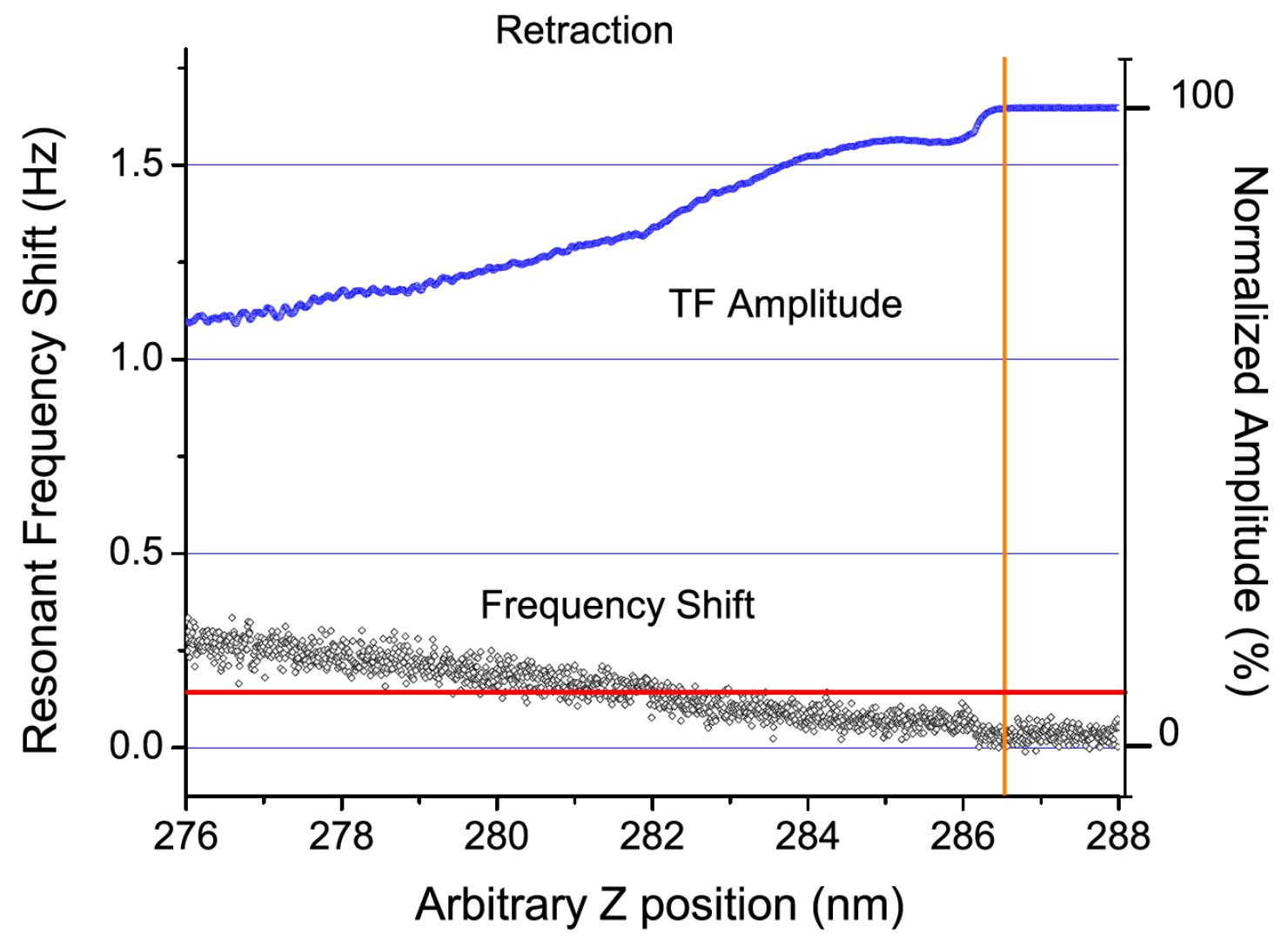

Figure 53. The retraction process of figure 52. The sudden change in the interaction after the retraction is very well defined.

The tip was brought above the polymer region and another approach and retraction curve was taken. Figure 54 shows the process. The limit for the approach is again selected as the $45 \%$ of the initial amplitude signal of the QTF. Again, the graph is divided in two regions for the approach and retraction processes. This time the two have similar lengths (approximately $19 \mathrm{~nm}$ ) and the beginning and end of both are not very well defined as in the case of silicon. 


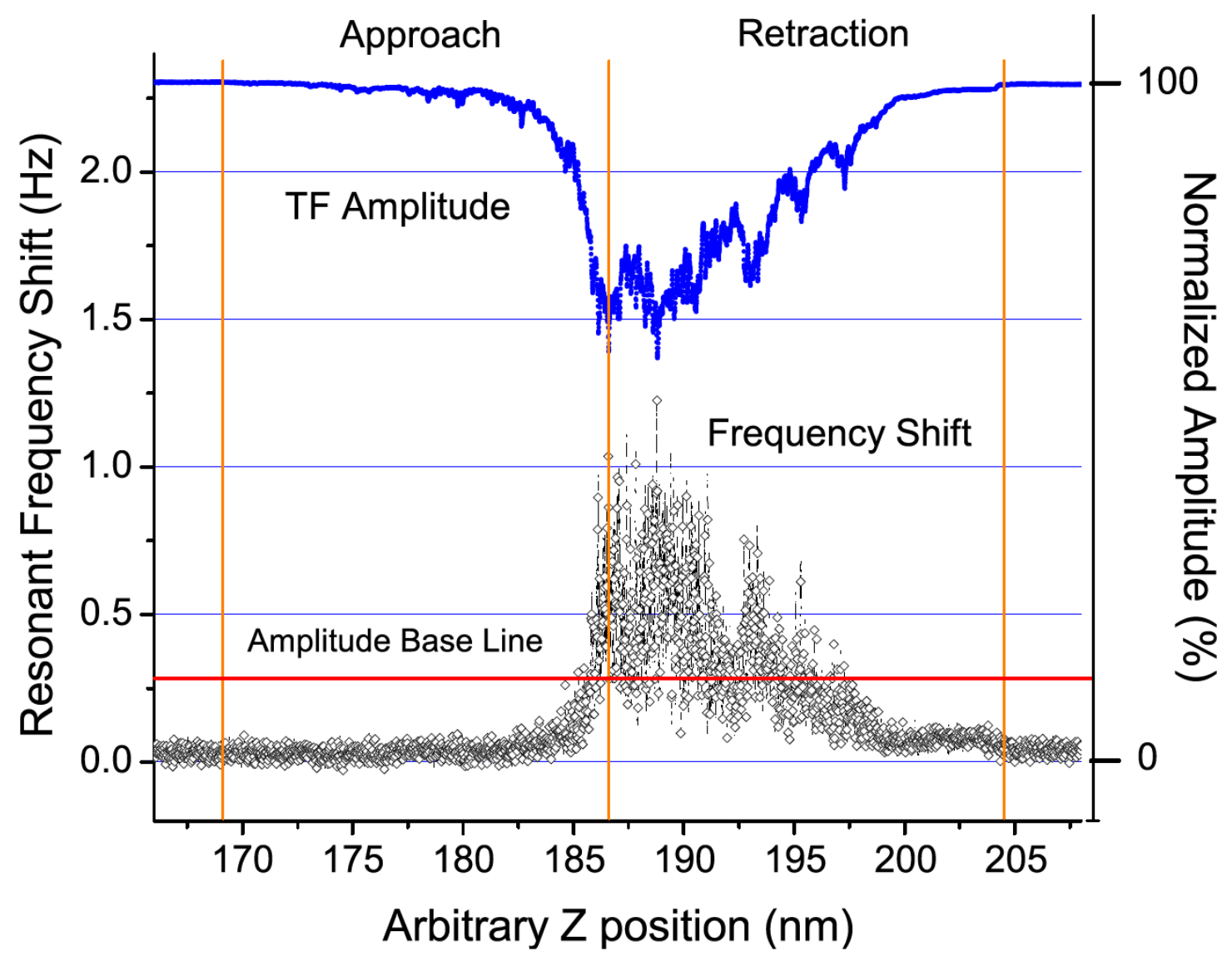

Figure 54. Approach and retraction curve for a $45 \%$ offset in polymer with a gold tip. The frequency shift curve has a trend line included to show the oscillations more clearly.

The beginning of the approach contains only a dissipative interaction and the conservative part only starts a $3 \mathrm{~nm}$ to $4 \mathrm{~nm}$ before the $45 \%$ limit is reached. The retraction region is $20 \mathrm{~nm}$ in length, similar to the case of silicon. It can be seen that interactions are less stable as the tip travels closer to the sample. 


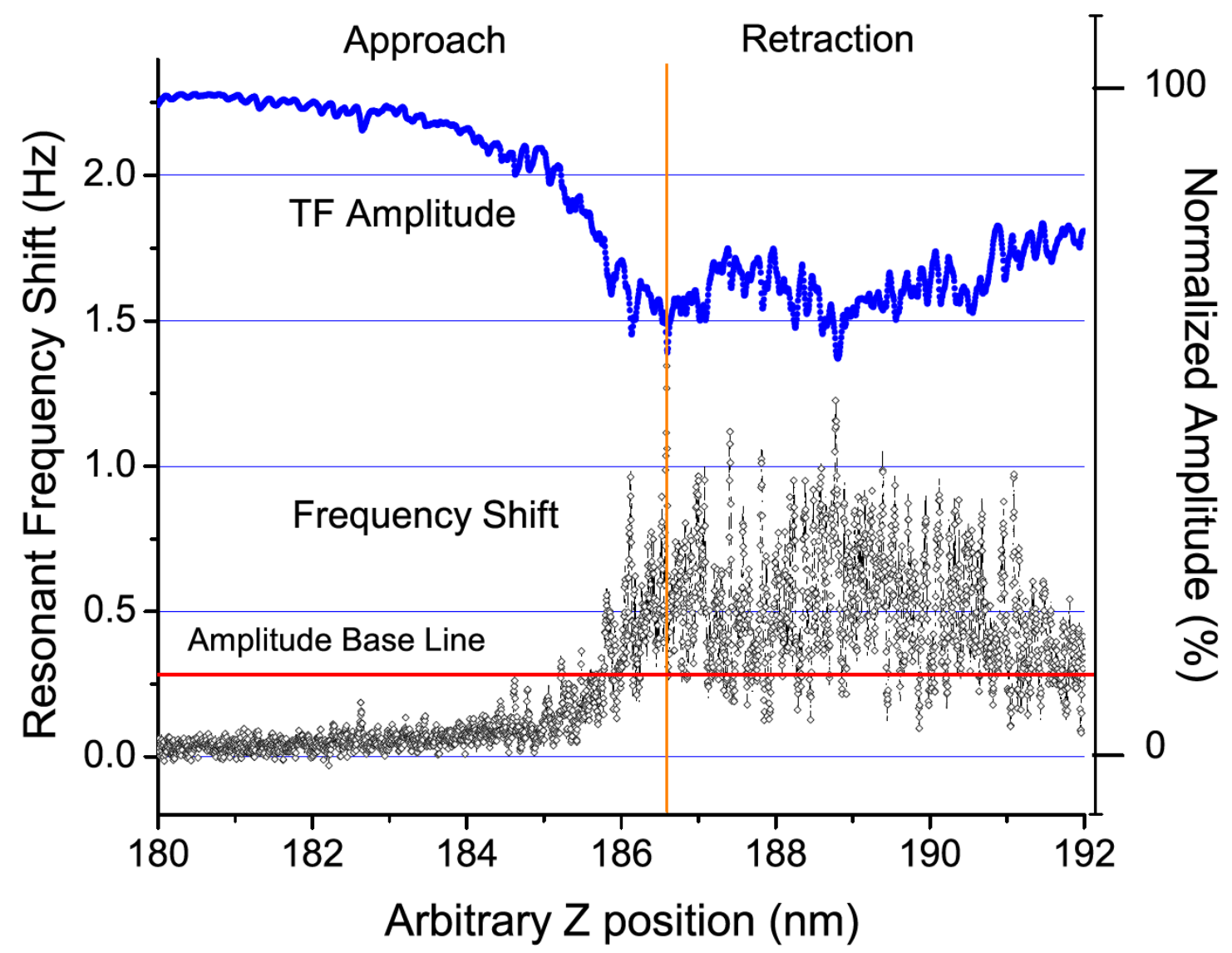

Figure 55. The approach process. It contains a region of pure dissipative interaction followed by one of both dissipative and conservative interactions.

The oscillations of both amplitude and resonant frequency shift are larger than those in the previous case, hinting at a more position-sensitive interaction. 


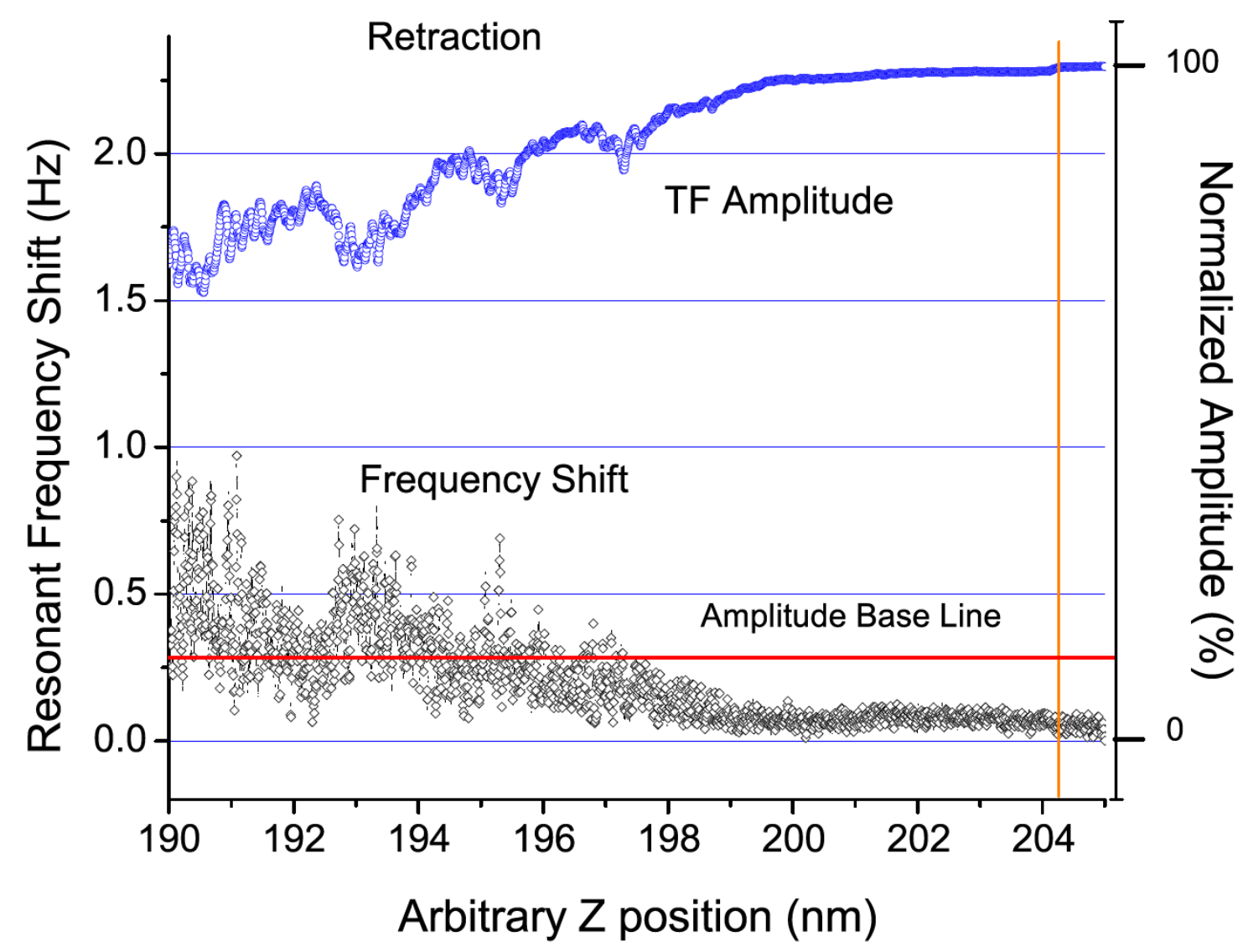

Figure 56. Retraction process. This region has the same length as observed over the silicon sample.

A straightforward experiment tested the behavior of the tip-layer-sample interactions with fixed position. The tip was maintained just above the interaction region, and was suddenly (step motion) approached in steps of $5 \mathrm{~nm}$ into the damping region as showed in 57. The entire process is shown in figure 58 and it can be divided in two approaches. A first approach and retraction process on the left of the graph and a second, lengthier one on the right. 

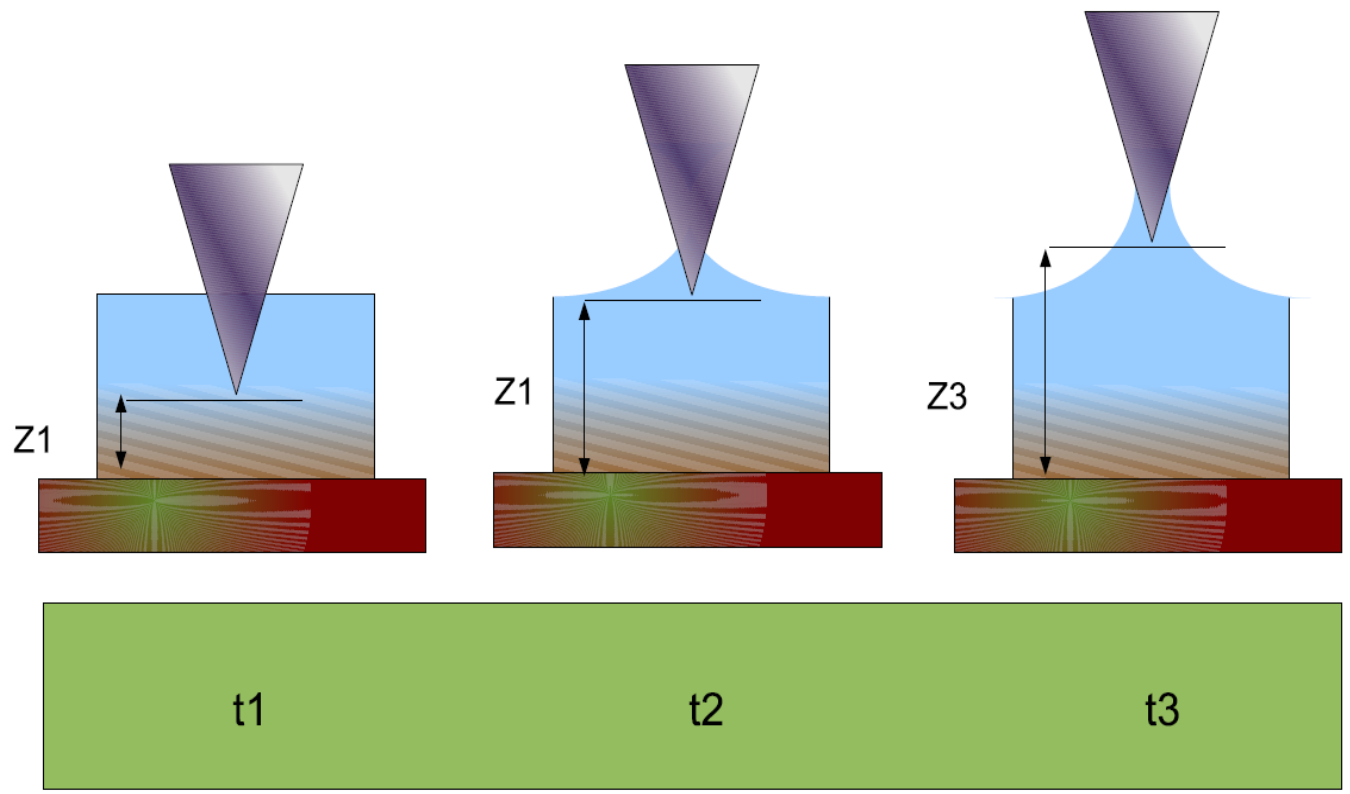

Figure 57. Step Motion of tip in water layer.

Following figure 58 the tip first approaches the surface and the amplitude decreases. Once the tip has made 'contact' and the tip motion has damped, it has to be retracted further away from the initial position as showed by the position curve, giving evidence of meniscus formation, and has to retract $15 \mathrm{~nm}$, in steps of $5 \mathrm{~nm}$, going back to a position $5 \mathrm{~nm}$ above the initial position to avoid interaction with the contamination layer. However, after retracting the additional $5 \mathrm{~nm}$ and leaving the tip standing still, the amplitude signal will decrease and follow and erratic behavior towards a strong conservative interaction as indicated by the increasing frequency shift. 


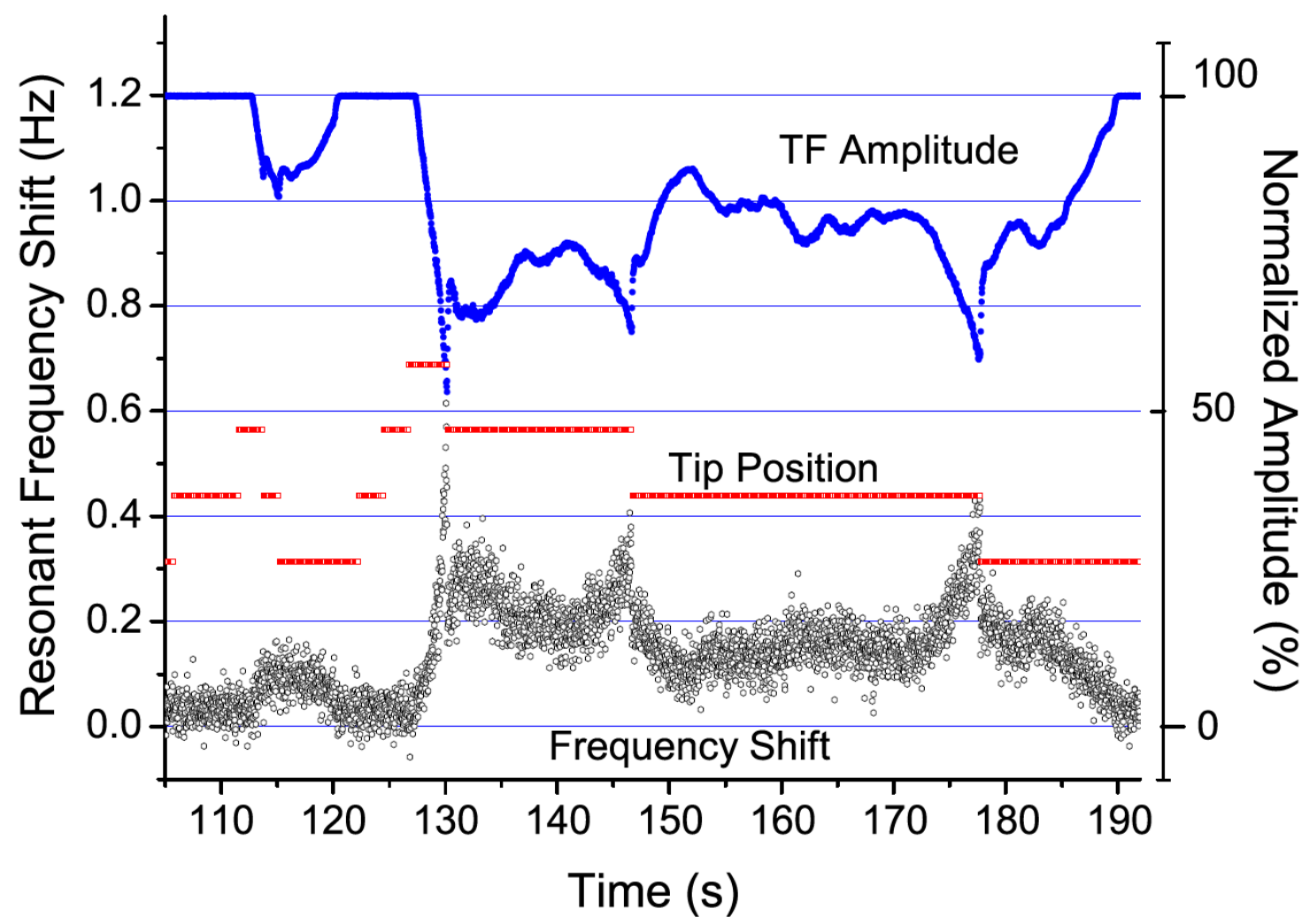

Figure 58. QTF amplitude stability on silicon experiment. Two different approach processes are observed (according to the amplitude curve).

Because of the size of the steps $(5 \mathrm{~nm})$ and observing this behavior without any apparent vertical motion of the tip, thermal drift appears to be the most probable cause given the fact that it affects every single part of the SPM head and can produce a significant vertical motion that would bring the tip closer to the surface. A variation of 0.001 degree centigrade in the temperature would produce a drift of approximately $1 \mathrm{~nm}$ in the $\mathrm{z}$ axis for aluminum and the corresponding dimensions of the SPM head. 


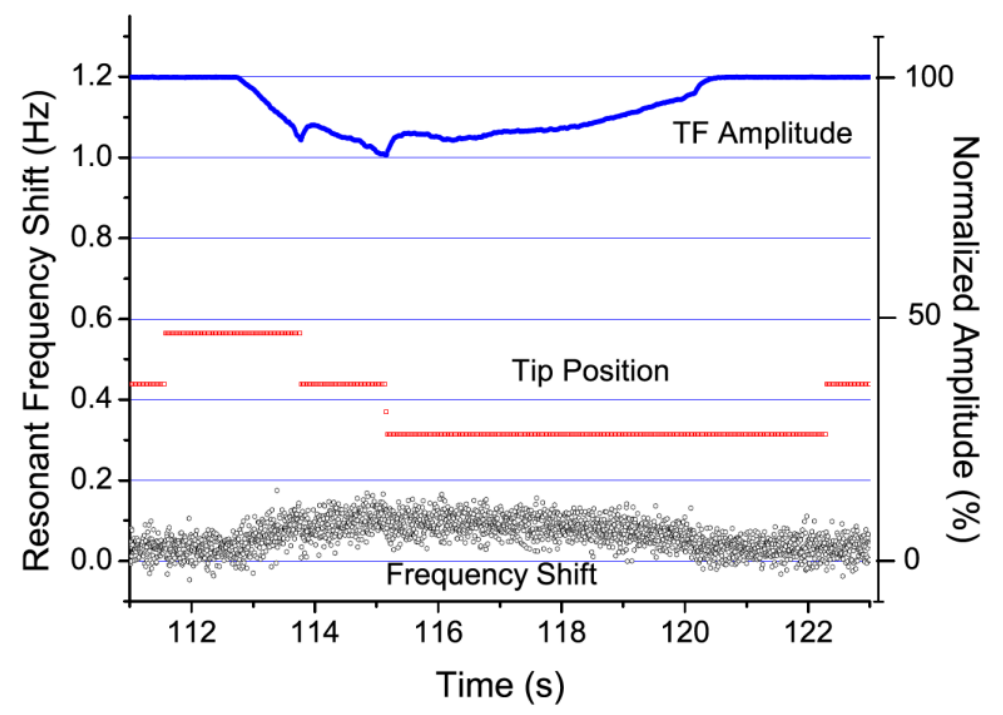

Figure 59. First attempt to approach. The amplitude signal decreases by $15 \%$. The resonant frequency shifts only $100 \mathrm{mHz}$.

Figures 59 and 60 show the first and second approach respectively and give an estimate of the frequency shifts for each process.

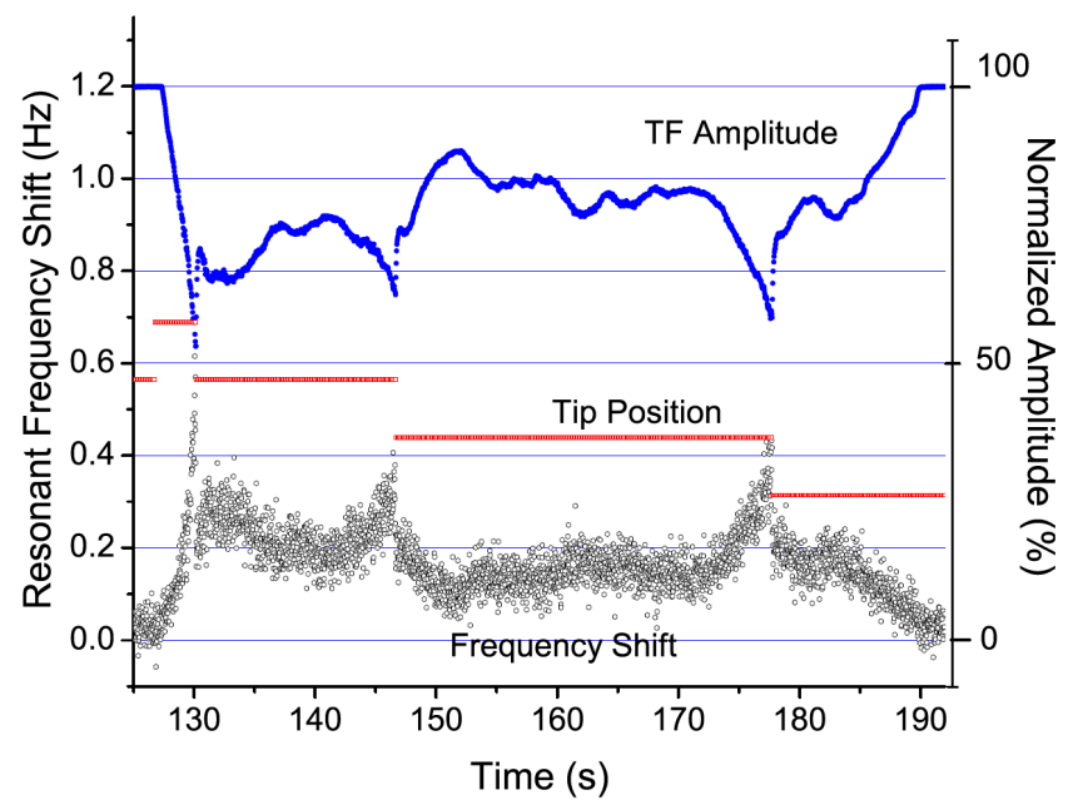

Figure 60. Second attempt. Amplitude decreases 50\% and the resonant frequency shifts $600 \mathrm{mHz}$. 


\subsection{Imaging with WGAS Feedback}

In order to test the USTS acoustic signal capabilities as a feedback signal, a calibration grid from Micromash (TGX series grating for lateral calibration, 10 um pitch.) was imaged. Previously, and for comparison purposes, an SEM image of the grid was taken (figure 61).

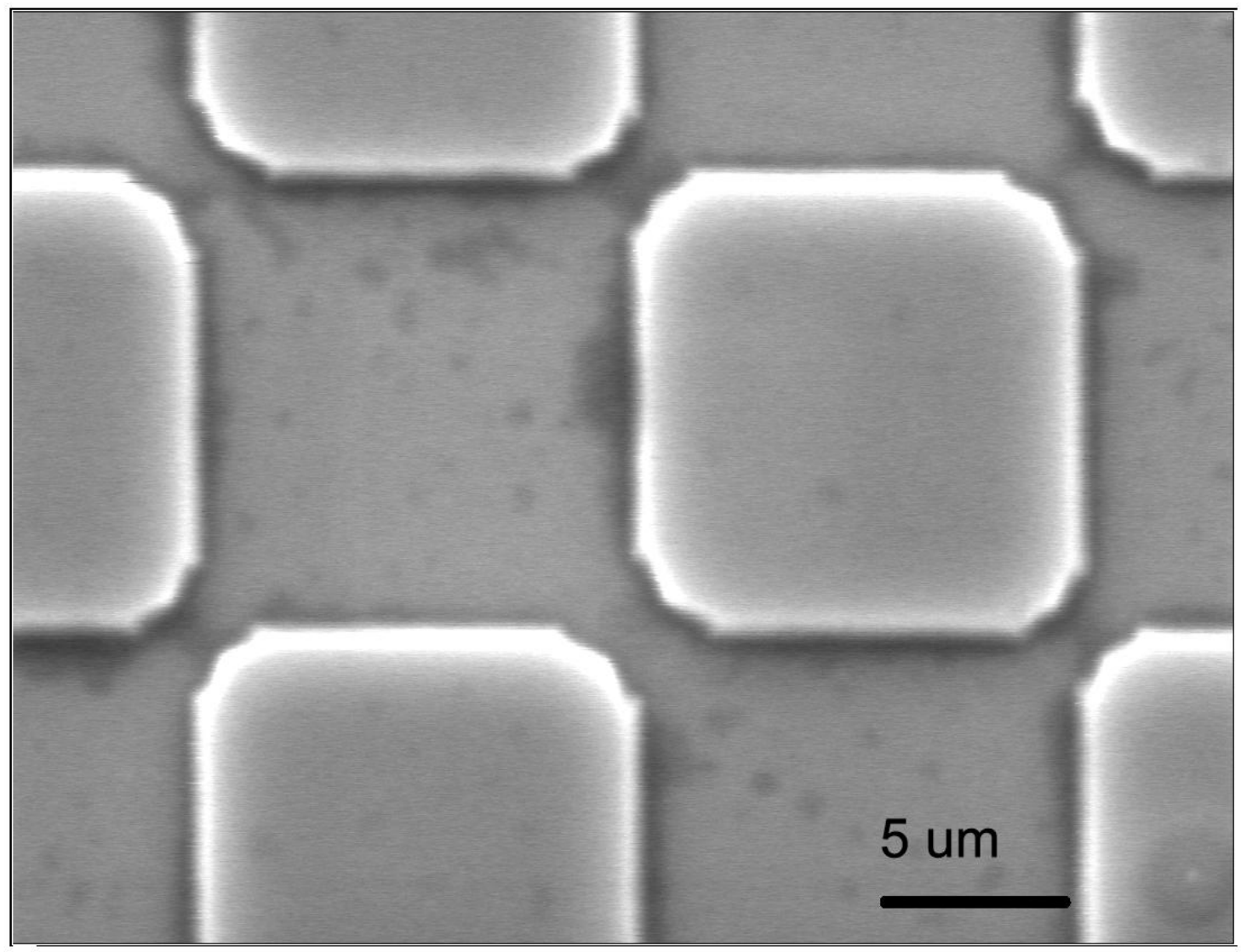

Figure 61. SEM image of calibration grid.

After proper cleaning, the sample is placed in the SPM sample holder and the WGAS feedback is turned on. The scanning speed was limited to less than 0.1 lines per second due to the use of the AM mode instead of PM or FM modes. The latter were impractical 
because of noise considerations explained in chapter 5 . The scan frequency of $350 \mathrm{~nm} / \mathrm{s}$ meant approximately $3 \mathrm{~ms}$ per nanometer motion. The amplitude of the QTF, calculated following the procedure in chapter 3 , had to be increased from the standard value of 2-5 $\mathrm{nm}$ to about 9-10 $\mathrm{nm}$ to generate the necessary acoustic energy to overcome the noise level and increase the SNR to an acceptable level (around 100).

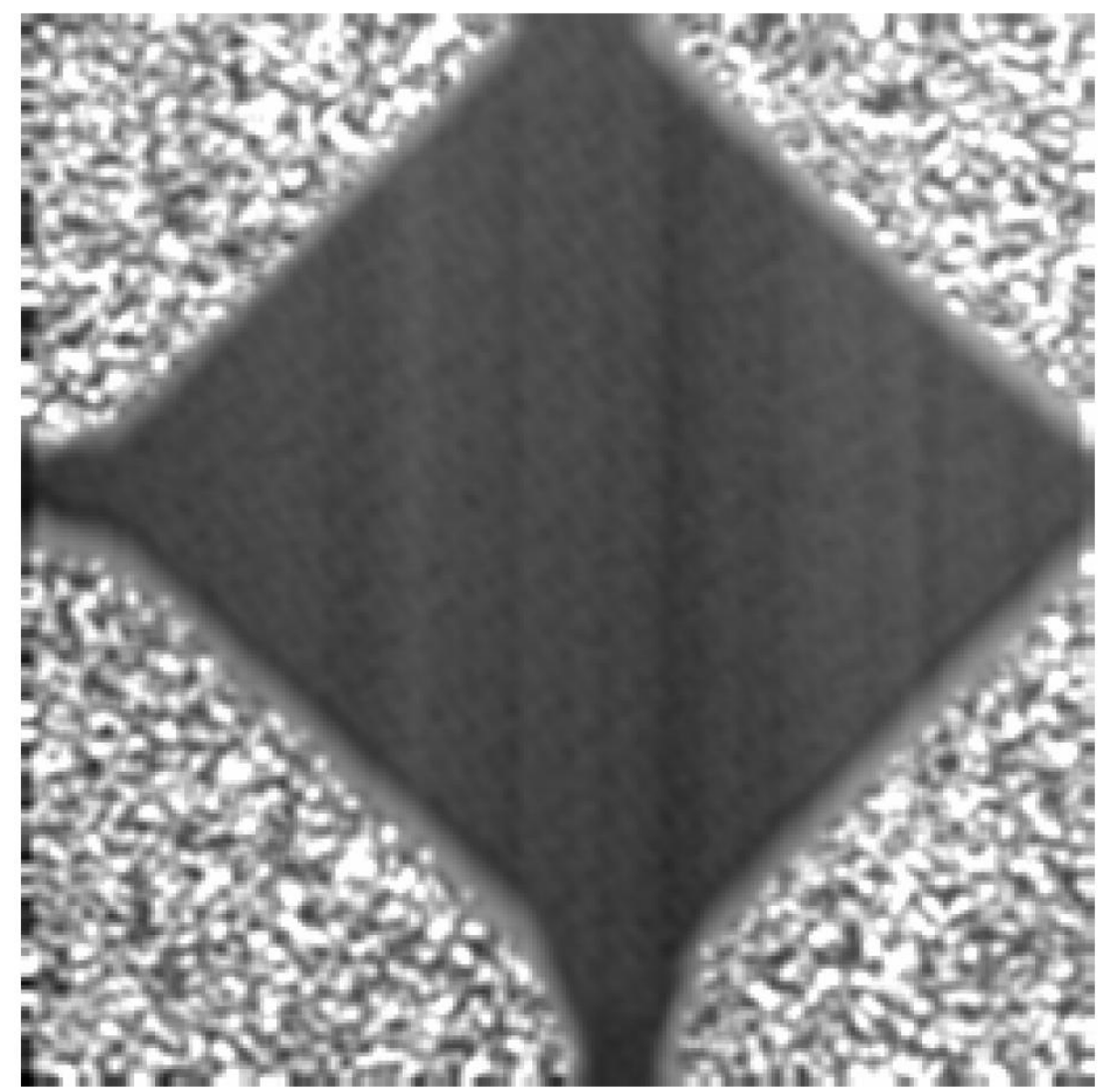

Figure 62. Preliminary image of calibration grid.

Both images in figures 62 and 63 show the same region but figure 63 has a cleaner signal and it required minimum filtering (FFT filtering). 

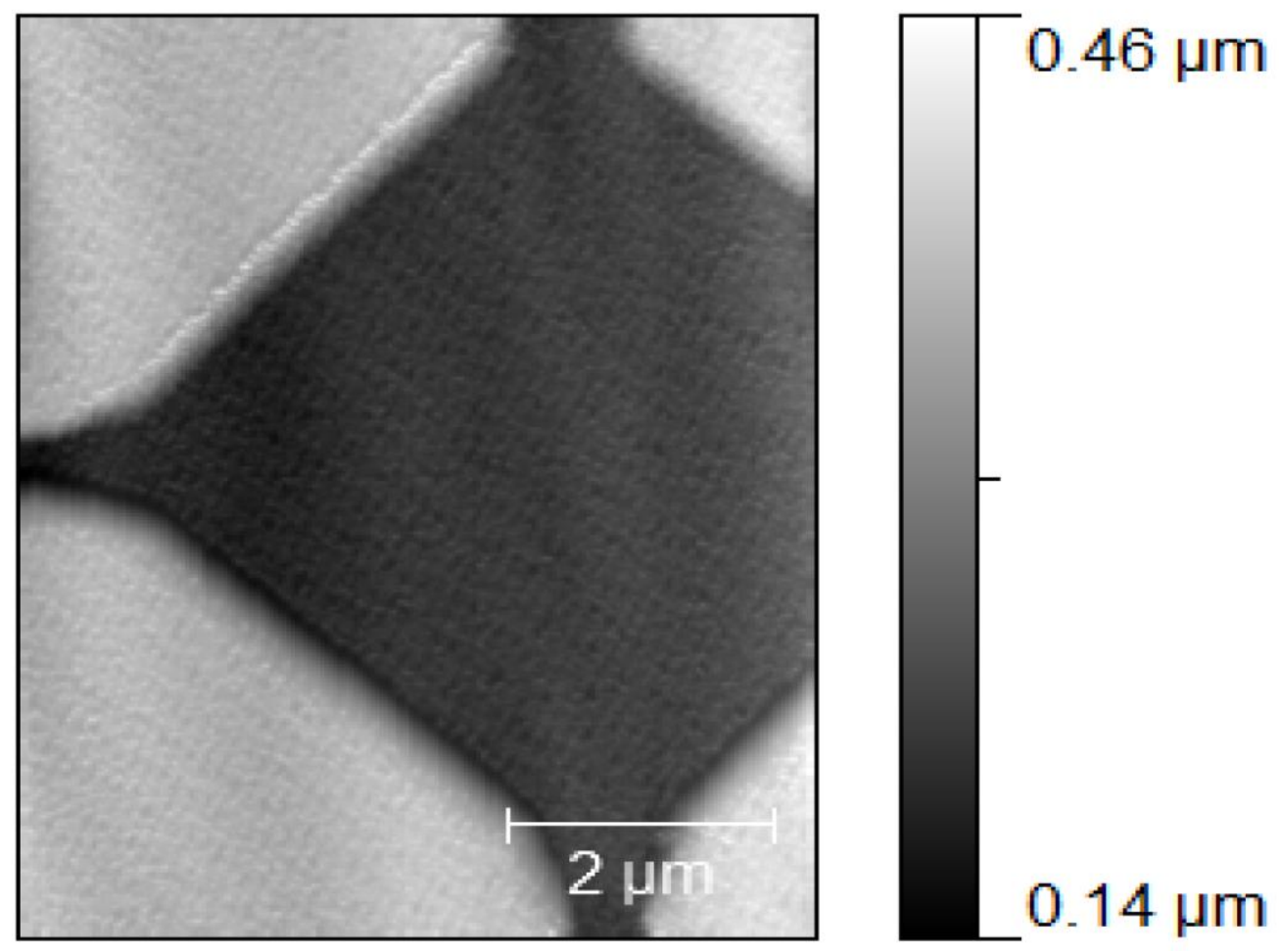

Figure 63. Image of Calibration grid using WGAS feedback.

Both images in figures 62 and 63 show the same region but figure 63 has a cleaner signal and it required minimum filtering (FFT filtering). The image processing was performed with the open source program Gwyddion [35]. An image with a greater range (in the order of $80 \mu \mathrm{m}$ ) with AM was attempted. The speed was improved compared to that of regular AM feedback mode, but the signal was too noisy to allow for FM feedback mode without endangering the tip. Nevertheless, the scan rate in FM feedback mode was improved by a factor of 4 . Further noise reduction and real time signal processing can improve this factor and increase the SNR. 


\section{Chapter 6}

\section{Conclusions}

Implementation of the Shear Force SPM with WGAS feedback in AM mode was accomplished. Several steps to improve the acoustic signal acquisition were taken and results were successful.

Theoretical and experimental evidence support Frequency Modulation (FM) as a valuable tool for faster scanning speeds when compared to amplitude modulation, as well as a better understanding of the forces involved in the tip sample interactions.

It was shown that the feedback with Whispering Gallery Acoustic Sensing (WGAS) can be used as a feedback technique in SPM, providing the advantage of directly measuring the mechanical oscillation of the QTF and possible signal amplification through resonant mode modification of the Whispering Gallery. Such improvements can be further studied and implemented in order to increase the signal to noise ratio (SNR) of the WGAS. 


\section{Chapter 7}

\section{Proposed Experiments}

\footnotetext{
Along with increased sensitivity and improved position determination, there are a few experiments that we can attempt with the system that has been assembled.
} 


\subsection{Subsurface Ultrasonic Holography}

Following the discussion presented in chapter 2 regarding the different acoustic techniques implemented in SPM, a natural consequence of the WGAS feedback technique is to take advantage of the SAWs formed on the sample's surface for imaging and characterization. A preliminary setup was implemented to measure the ultrasonic oscillations originating on the tip and channeled through the surface (as a result of the tipsample interaction), traveling through the sample and measured by the USBS. The phase provided by the USBS has information of the internal structure because the phase shift is related to the different mediums the wave travels through, as long as the phase of the excitation and the QTF piezo response are kept in phase. To obtain nanometer resolution with this technique, as explained in section, it is necessary to have signals in the order of Megahertz. Equation (30) shows the calculation for a $1 \mathrm{~nm}$ depth resolution. For a change in phase in the order of 1 degree per $1 \mathrm{~nm}$ of space change the following relations hold,

$$
\frac{x}{\lambda}=\frac{t}{T}=\frac{1}{360}
$$

where $f$ is the frequency of the vibration, $\lambda$ is the wavelength of sound in the material which depends on the frequency of the excitation (since the velocity is constant for a given material). Using $x=1 \mathrm{~nm}, \lambda=360 \mathrm{~nm}$ a suitable resolution of about $1 \mathrm{~nm}$ per 
degree of phase shift is obtained. Next, this value is used to find the frequency necessary to reach this resolution and using $\boldsymbol{v}=\mathbf{2 2 0 0} \mathbf{m} / \mathbf{s}$ for silicon in the equation

$$
f=\frac{v}{\lambda}
$$

The result is $6 \mathrm{GHz}$ Here is where the non-linear behavior of the forces on the tip play a role of mechanical diode. For this reason, lower frequencies can be used, in the order of Megahertz. A preliminary result is presented in figure 64. A sample with irregular features was imaged, its topography (left image), recorded simultaneously with the magnitude and phase of the acoustic signal (the ultrasonic transducer located at the bottom of the sample). The phase image clearly shows distinct features in regions where topography is simply flat.

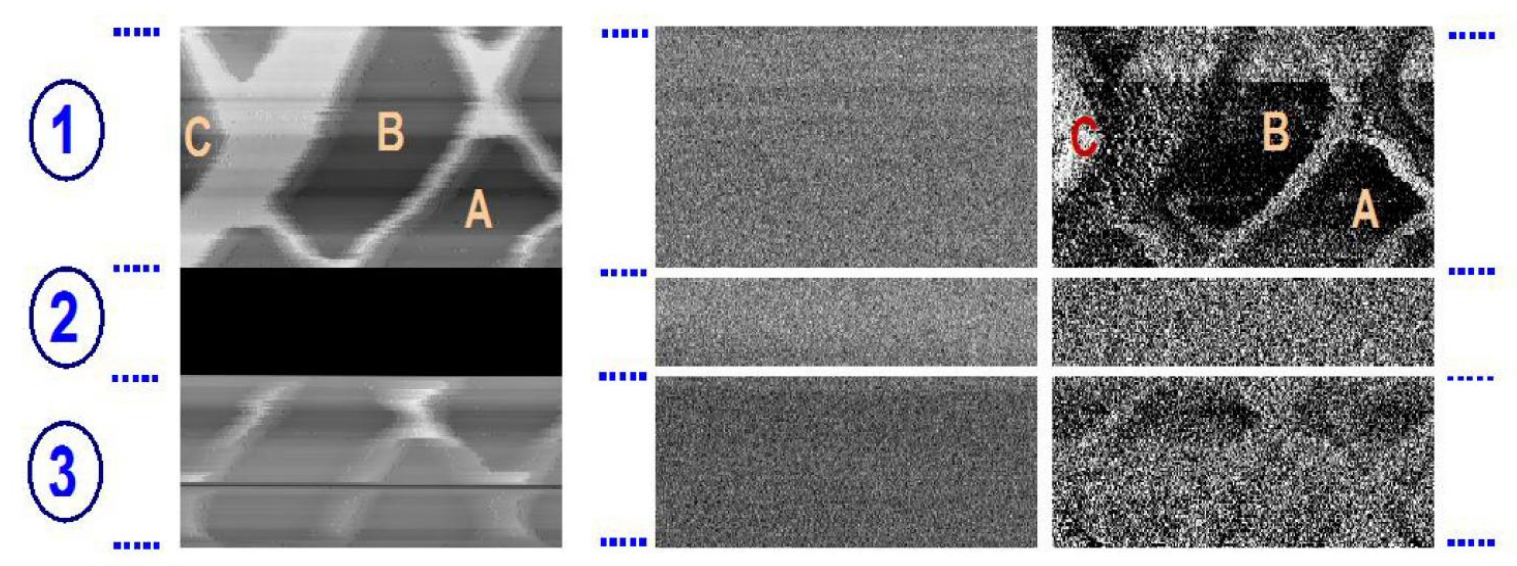

Figure 64. Ultrasonic subsurface preliminary result. 


\subsection{Integrated Near and Far Field Microscopy}

The implementation of a system able to further overcome the diffraction limit is being pursued. By quenching the surroundings and minimizing the interaction with the center part of a group of fluorophores, the determination of the center of its Point Spread Function (PSF) in the far field image, gains precision. In this technique it is crucial to have absolute control of the tip sample distance and good understanding of the interactions involved.
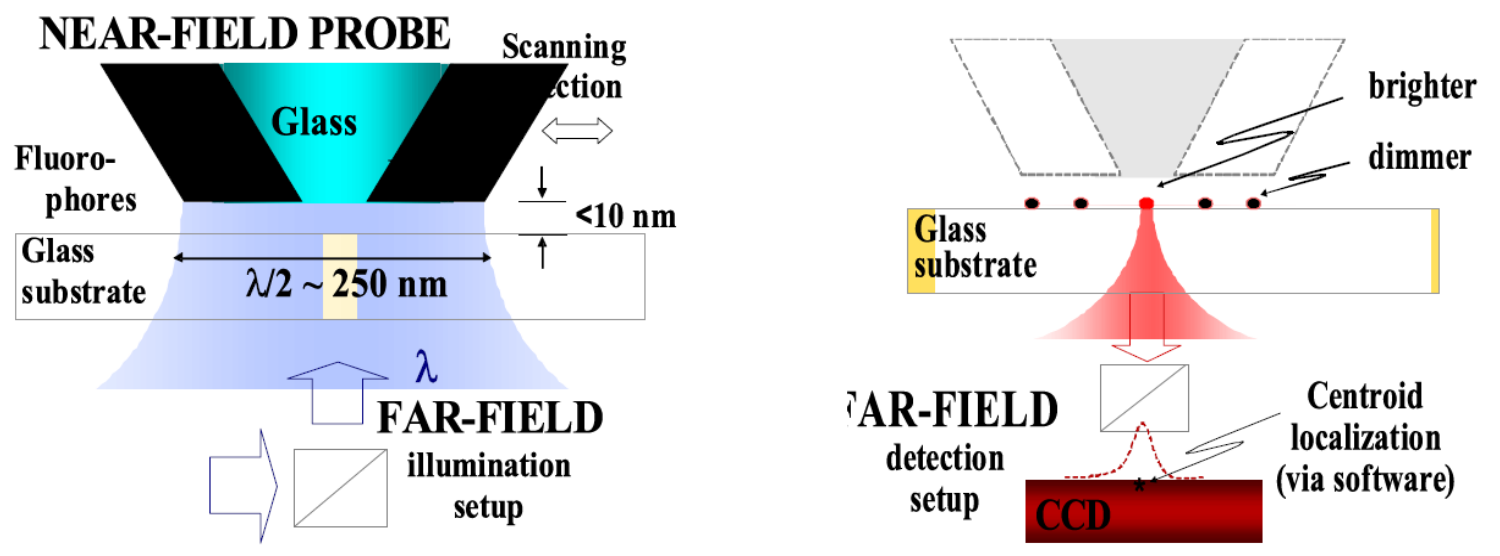

Figure 65. Integrated Near and Far Field Optical Microscopy (INFO) proposed technique.

This proposed technique uses a long known effect observed in NSOM (figure 65). The interaction between the coated metal fiber tip quenching fluorophores. Several papers have been published that discuss the processes causing this quenching $[69,70,71]$. Bringing a metallic object close to a fluorescent molecule will modify its absorption and emission properties. Depending on the distance to the molecule, the radiative and nonradiative dynamics can be modeled as either oscillations with distance as a result of 
interference between the radiated and reflected emission of the fluorophore radiation field when the distance is larger than $10 \mathrm{~nm}$ or show a strong dependence $\mathrm{d}^{-3}$ or $\mathrm{d}^{-4}$ for smaller distances. The excitation of electron-hole pairs and the induction of currents in the metal surface are the channels for non radiative relaxation transfers [71].

The localization technique calculates the position of an object by finding the center of its Point Spread Function (PSF). When the fluorescent molecules are far away from each other (several lengths the emission wavelength) the position can be calculated with any desired precision since a larger collection of photons is needed. However, when the molecules are closer together (in the order of the emission wavelength), the spatial resolution decreases and a method to distinguish two point-like sources from what appears to be one.

The localization of an individual fluorophore depends only on the number of collected photons and the calculation of the center positions of the PSF is limited by Abbe's Principle: the determination of two point like sources can only be made as long as there is no overlapping between their Full Widths at Half Maximum (FWHM). It follows that finding a way to suppress (quench) the emission of surrounding fluorophores will improve the localization precision. The system would incorporate an standard Inverted microscope (Olympus IX71), NSOM tips, extra optical setup for Total Internal Reflection illumination (TIRF) and a homemade platform to accommodate the SPM shear force system on top of the IX71. 


\section{References}

[1] G. Binning, H. Rohrer, Ch. Gerber, and E. Weibel, Phys. Rev. Lett., vol. 49, p. 57 (1982).

[2] G. Binning, C.F. Quate and Ch. Gerber, Phys. Rev. Lett, vol. 56, p.930 (1986).

[3] A. H. La Rosa, R. Nordstrom, X. Cui, J. McCollum, Rev. Sci. Instrum., vol. 76, p. 093707 (2005).

[4] A. H. La Rosa, N. Li, and K. Asante, Invited Paper in "Nanofabrication: Technologies, Devices, and Applications II", Warren Y. Lai, L. E. Occola, Stanley Pau Eds., Symposium in Optics East 2005, Boston, MA. Proc. SPIE 6002, pp. 163-170 (2005).

[5] A. Lewis, M. Isaacson, A. Harootunian, and A. Murray, Ultramicroscopy, vol. 13, p. 227 (1984).

[6] D.W. Pohl, W. Denk, and M. Lanz, Appl. Phys. Lett., vol. 44, p. 651 (1984).

[7] Y. Martin and K. Wickramasinghe, Appl. Phys. Lett. vol. 50, p. 1455-1457 (1987).

[8] Martin Y, Abraham DW, Wickramasinghe HK, Appl. Phy.s Lett., vol. 52, p. 1103 (1988).

[9] P. M. Bridger, Z. Z. Bandic, E. C. Piquette, and T. C. McGill, Appl. Phys. Lett., vol. 74, 3522 (1999).

[10] Dürig U., Züger O., Stadler A., J. Appl. Phys., vol. 72, p. 1778 (1992).

[11] Toshio Ando, Takayuki Uchihashi, Takeshi Fukuma, Progress in Surface 
Science, vol. 83, pp. 337-437 (2008).

[12] Lemons, R. A. and Quate, C. F., Appl. Phys. Lett., vol. 24, pp. 163-165 (1974).

[13] O. V. Kolosov and K. Yamanaka, Jpn. J. Appl. Phys., vol. 32, p. 22 (1993).

[14] O. V. Kolosov, M. R. Castell, C. D. Marsh, G. A. D. Briggs, T. I. Kamins, R. S. Williams, Phys. Rev. Lett., vol. 81 (5), pp. 1046-1049 (1998).

[15] Rabe U. and Arnold W., Appl. Phys. Lett., vol 64, p. 1493 (1994).

[16] Chilla E., Hesjedahl T., Fröhlich H.-J. Phys. Rev. B, vol 55, p. 15852, (1997).

[17] Yamanaka K. and Nakano S., Jpn. J. Appl. Phys., vol 35, p. 3787 (1996).

[18] Roman Gr. Maev, "Acoustic Microscopy: Fundamentals and Applications", WILEY-VCH Verlag GmbH \& Co. KGaA, Weinheim, (2008).

[19] P. Günther, U. Ch. Fischer, and K. Dransfeld. "Scanning Near Field Acoustic Microscopy". Appl. Phys. B, vol. 48, p. 89-92 (1989).

[20] K. Karrai and R.D. Grober, Appl. Phys. Lett., vol. 66, p. 1842 (1995).

[21] A. G. Ruiter, J. A. Veerman, K. O. van der Werf, and N. F. van Hulst., Appl. Phys. Lett., Vol. 71(1), p. 28-30 (1997).

[22] A. G. T. Ruiter, J. A. Veerman K. O. van der Werf, M. F. Garcia-Parajo, W. H. J. Rensen, and N. F. van Hulst, Ultramicroscopy, vol. 71, p. 149-157 (1998).

[23] W. A. Atia and C. C. Davis, Appl. Phys. Lett., Vol. 70(4), pp. 405-407 (1997).

[24] Yongho Seo and Wonho Jhe, Rep. Prog. Phys., vol. 71, p. 016101, (2008).

[25] K. S. Van Dyke, Phys. Rev., vol. 25, p. 895 (1925).

[26] D. W. Dye, "The piezo-electric quartz resonator and its equivalent electrical circuit ", Proc. Phys. Soc. London, vol. 38, p. 399-458 (1925).

[27] K. S. Van Dyke, Proceedings of the 1928 IEEE International Frequency Control 
Symposium, New York, vol. 16, p. 742 (1928).

[28] J. Rychen. "Combined Low-Temperature Scanning Probe Microscopy and Magneto-Transport Experiments for the Local Investigation of Mesoscopic Systems". PhD thesis, Swiss Federal Institute of Technology ETH, 2001. Chapter 4.

[29] J. Rychen, T. Ihn, P. Studerus, A. Herrmann, and K. Ensslin, Rev. Sci. Instrum., vol 71 (4), p. 1695, (2000).

[30] A. Lazarev, M. Fang, Q. Luo, and X. Zhang. "Formation of fine near field scanning optical micrscopy tips: Part 1 . By static and dynamic chemical etching ”. Rev. Sci. Instrum., vol. 74 (8), pp. 3679-3683 (2003).

[31] A. Lazarev, M. Fang, Q. Luo, and X. Zhang. "Formation of fine near field scanning optical micrscopy tips: Part 2. By laser - heated pulling and bending ”. Rev. Sci. Instrum., vol. 74 (8), pp. 3684-3688 (2003).

[32] Justin J. McCollum, "Probe Fabrication for Near-Field Optical Microscopy Application", Master of Science in Physics Thesis, Portland State University. Portland, Oregon, (2005).

[33] Kofi Adu Asante, "Fabrication of Tungsten Tips for Shear-Force Microscopy and its Applications ", Master of Science in Physics Thesis, (2007).

[34] Xiquan Cui, "Near Field Aocustic Microscope and its Application in Nanocharacterization", Master of Science in Physics Thesis, Portland State University. Portland, Oregon (2005).

[35] Petr Klapetek, David Nečas, Christopher Anderson (2010). Gwyddion - Free SPM Data Analysis Software, GNU General Public License. http://gwyddion.net/. 
[36] Best, Roland E."Phase Lock Loops: Design, Simmulation and Applications", (6th edition), McGraw-Hill.

[37] Khaled Karrai, Robert D. Grober, SPIE, Vol. 2535 p. 69 (1995).

[38] A. Castellanos-Gomez, N. Agraï, and G. Rubio-Bollinger, Nanotechnology, vol. 20, p.215502 (2009).

[39] T.R. Albrecht, P. Grütter, D. Horne and D. Rugar, J. Appl. Phys., vol. 69 (2), pp. 668-673 (1991).

[40] M. Labardi, Nanotechnology, vol. 18, p. 395505, (2007).

[41] Xiquan Cui and Andres La Rosa, Appl. Phys. Lett., vol. 87, p. 231907 (2005).

[42] John E. Sader, and Suzanne P. Jarvis, Physical Review B, vol. 74, p. 195424 (2006).

[43] Manhee Lee, Junghoon Jahng, Kyungho Kim, and Wonho Jhea, Appl. Phys. Lett., vol. 91, p. 023117 (2007).

[44] J.-P. Ndobo-Epoya,b, E. Lesniewskaa, J.-P. Guicquerob, Ultramicroscopy, vol. 103, pp. 229-236 (2005).

[45] J. M. Friedt, E. Carry, Z. Sadani, B. Serio, M. Wilm, S. Ballandras, "Quartz tuning fork vibration amplitude as a limitation of spatial resolution of shear force microscopes", FEMTO-ST, 32 avenue de l'Observatoire, 25040 Besan,con Cedex, France.

[46] "Standard Practice for Secondary Calibration of Acoustic Emission Sensors", ASTM-E1781-96, ASTM E1781 (Nondestructive Testing Standards).

[47] John E Sader, Takayuki Uchihashi, Michael J Higgins, Alan Farrell, Yoshikazu Nakayama and Suzanne P Jarvis, Nanotechnology, vol. 16, p. S94-S101 (2005). 
[48] U. Durig, IBM Research Division, Zurich Research Laboratory, CH-8803 Rüschlikon, Switzerland, Appl. Phys. Lett., vol. 75 (3), (1999).

[49] S. H. Ke and T. Uda,K. Terakura, Phys. Rev. B, vol. 59 (20), pp. 13267-13272 (1999).

[50] Franz J. Giessibl and Hartmut Bielefeldt, Phys. Rev. B, vol. 61 (15), pp.9968$9971(2000)$.

[51] J. P. Aimé, R. Boisgard, L. Nony, and G. Couturier, Phys. Rev. Lett., vol. 82 (17), p.3388 (1999).

[52] H. Hölscher, B. Gotsmann, W. Allers, U. D. Schwarz, H. Fuchs and R. Wiesendanger, Physical Review B, vol. 64, p. 075402 (2001).

[53] U. Durig, New Journal of Physics, vol. 2, pp. 5.1-5.12 (2000).

[54] R. Boisgard a, D. Michel b, J.P. Aimé,Surface Science, vol. 401, pp. 199-205 (1998).

[55] B. Anczykowski, B. Gotsmann, H. Fuchs, J.P. Cleveland, V.B. Elings, Applied Surface Science, vol. 140, pp. 376-382 (1999).

[56] Manhee Lee and Wonho Jhe, Phys. Rev. Lett., vol. 97, p. 036104 (2006).

[57] T. R. Albrecht, P. Grtitter, D. Horne, and D. Rugar, J. Appl. Phys., vol. 69 (2), p. 15 (1991).

[58] M. A. Lantz, et al. Science, vol. 291, p. 2580 (2001);

[59] Manhee Lee, Junghoon Jahng, Kyungho Kim, and Wonho Jhe, Appl. Phys. Lett., vol. 91, p. 023117 (2007).

[60] Yexian Qin, R. Reifenberger, Rev. Sci. Instrum., vol. 78, p. 063704 (2007).

[61] Junghoon Jahng, Manhee Lee, Hanheol Noh, Yongho Seo, and Wonho Jhe, Appl. 
Phys. Lett., vol. 91, p. 023103 (2007).

[62] SE40-Q, Low Frequency Transducer Q Series, Acoustic Emission Sensors, DECI Inc.

[63] SE25-P,Dual Performance Miniature Sensor, P Series, Acoustic Emission Sensors, DECI Inc.

[64] Boon PingNg, YingZhang, ShawWeiKok, Yeng Chai Soh, Ultramicroscopy, vol. 109, pp. 291-295 (2009).

[65] Raoul Stoöckle, Christian Fokas, Volker Deckert, and Renato Zenobi, Appl. Phys. Lett., vol. 75 (2), pp. 151-304 (1999).

[66] The SRanger Linux Team, "Signal Ranger DSP (SR-STD/-SP2 and SR-Mk2 with SR2-A810) Linux Kernel Module Support and DSP Tools”, 2002-2009, http://sranger.sourceforge.net.

[67] R. Bitter, T. Mohiuddin, M. Nawrocki. "LabVIEW Advanced Programming Techniques”, CRC Press, Taylor \& Francis Group, 2001.

[68] S. Sumathi,Surekha. P, "LabVIEW based Advanced Instrumentation Systems", Springer-Verlag Berlin Heidelberg, (2007).

[69] B.N.J Person and N.D. Lang Phys. Rev. B 26, 5409 (1982).

[70] G. Cnossen, K.E. Drabe, D.A Wiersma, J. Chem Phys,98 5276 (1993).

[71] J. Phys. C: Solid State Phys., Vol. 11,4251-4269, (1978). 